University of Louisville

ThinkIR: The University of Louisville's Institutional Repository

Electronic Theses and Dissertations

8-2013

\title{
Olanzapine-induced liver injury : direct metabolic effects, exacerbation by high-fat diet, and protection with sulforaphane.
}

Robin H. Schmidt 1984-

University of Louisville

Follow this and additional works at: https://ir.library.louisville.edu/etd

\section{Recommended Citation}

Schmidt, Robin H. 1984-, "Olanzapine-induced liver injury : direct metabolic effects, exacerbation by highfat diet, and protection with sulforaphane." (2013). Electronic Theses and Dissertations. Paper 1272. https://doi.org/10.18297/etd/1272

This Doctoral Dissertation is brought to you for free and open access by ThinkIR: The University of Louisville's Institutional Repository. It has been accepted for inclusion in Electronic Theses and Dissertations by an authorized administrator of ThinkIR: The University of Louisville's Institutional Repository. This title appears here courtesy of the author, who has retained all other copyrights. For more information, please contact thinkir@louisville.edu. 


\title{
OLANZAPINE-INDUCED LIVER INJURY: DIRECT METABOLIC EFFECTS, EXACERBATION BY HIGH-FAT DIET, AND PROTECTION WITH SULFORAPHANE
}

\author{
By \\ Robin H. Schmidt \\ B.S., St. John's University, 2006 \\ M.S., University of Louisville, 2011

\begin{abstract}
A Dissertation
Submitted to the Faculty of the

School of Medicine of the University of Louisville

In Partial Fulfillment of the Requirements

for the Degree of
\end{abstract} \\ Doctor of Philosophy \\ Department of Pharmacology and Toxicology \\ University of Louisville \\ Louisville, KY
}

August 2013 



\title{
OLANZAPINE-INDUCED LIVER INJURY: DIRECT METABOLIC EFFECTS, EXACERBATION BY HIGH-FAT DIET, AND PROTECTION WITH SULFORAPHANE
}

\author{
By \\ Robin H. Schmidt \\ B.S., St. John's University, 2006 \\ M.S., University of Louisville, 2011
}

A Dissertation approved on July 8, 2013

by the following Committee:

Gavin E. Arteel, Ph.D. (Mentor)

Frederick W. Benz, Ph.D.

Aruni Bhatnagar, Ph.D.

Russell A. Prough, Ph.D.

J. Christopher States, Ph.D. 


\section{DEDICATION}

For Joe, who finds the poetry in science 


\section{ACKNOWLEDGMENTS}

This dissertation would not be possible without the knowledge, patience, and encouragement of my mentor, Dr. Gavin Arteel. I am also deeply grateful to my committee members, whose scientific and professional insights have been invaluable to my growth as a researcher. 


\title{
ABSTRACT \\ OLANZAPINE-INDUCED LIVER INJURY: DIRECT METABOLIC EFFECTS, EXACERBATION BY HIGH-FAT DIET, AND PROTECTION WITH SULFORAPHANE
}

\author{
Robin H. Schmidt \\ 07/08/2013
}

Olanzapine $(\mathrm{OLZ})$ is an effective first-line treatment for schizophrenia and bipolar disorder. The benefits of OLZ are countermanded by side effects such as weight gain, glucose intolerance, dyslipidemia, and liver injury. These effects impact not only antipsychotic medication compliance, but also increase the health risks to patients. Most studies to date have focused on potential effects of OLZ on the central nervous system (e.g., hypothalamic regulation of satiety); however, peripheral changes in key metabolic organs such as the liver may also play a critical role. The obesity rates in the US are now at epidemic levels and obesity-induced liver disease (i.e., non-alcoholic fatty liver disease, or NAFLD) is on the rise. It is now understood that obesity is a significant risk factor for a myriad of drug-induced liver injuries. Given that the obesity incidence in the psychiatric population is even higher than in the US population as a whole, the effects of OLZ may exacerbate an underlying condition in these patients. 
The purpose of this work was to determine the mechanisms of OLZinduced hepatic dysmetabolism, and to test the hypothesis that OLZ enhances obesity-induced hepatic injury. OLZ was administered in a mouse model for four weeks along with a high-fat diet (HFD) or low-fat control diet. OLZ alone increased body weight and caused mild glucose intolerance, without a commensurate increase in food consumption. OLZ alone also caused hepatic steatosis and injury. Interestingly, although OLZ increased hepatic triglyceride synthesis and storage, it did not increase the synthesis or abundance of hepatic free fatty acids. OLZ administration appeared to cause a pseudo-fasted state and dramatically depleted hepatic glycogen reserves. These effects of OLZ occurred in parallel to significant changes in hepatic metabolite profiles. The protein kinases AMPK and mTOR are generally differentially activated, and mediate opposing metabolic functions; however, OLZ administration simultaneously activated both AMPK and mTOR. When OLZ and HFD were combined, there was an even greater increase in weight gain and glucose intolerance. Liver damage from concurrent HFD and OLZ was worse than liver damage resulting from HFD or OLZ alone.

Lastly, sulforaphane (SFN) was tested as a possible preventive against HFD- and OLZ-induced toxicity. Some mice receiving HFD and OLZ were also treated with SFN $(90 \mathrm{mg} / \mathrm{kg} / \mathrm{d})$. SFN, a known inducer of the Nrf2 intrinsic antioxidant pathway, prevented weight gain and liver injury and rescued hepatic glycogen storage. Furthermore, SFN decreased the presence of 4hydroxynonenal (4HNE) adducts in liver (>20-fold), indicating that SFN treatment 
substantially limits oxidative stress in this model.

In summary, these data show that OLZ dysregulates glucose and lipid metabolism and exacerbates hepatic changes caused by HFD exposure. The outcomes of OLZ administration on hepatic metabolism may reflect, in part, the contradictory inputs of simultaneous AMPK and mTOR activation. These data indicate that the metabolic changes caused by OLZ may sensitize the liver to injury caused by HFD and that underlying obesity/liver disease may aggravate OLZ-induced side effects. The activation of intrinsic antioxidant defenses with SFN can partially prevent these effects of OLZ and may represent a useful strategy to protect against liver injury. 


\section{TABLE OF CONTENTS}

\section{PAGE}

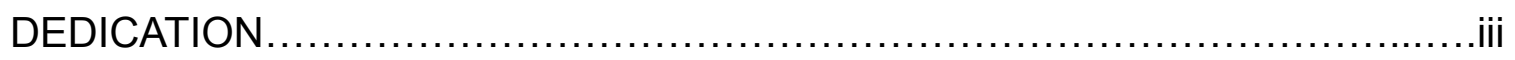

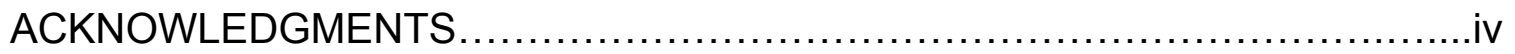

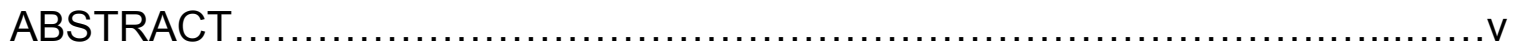

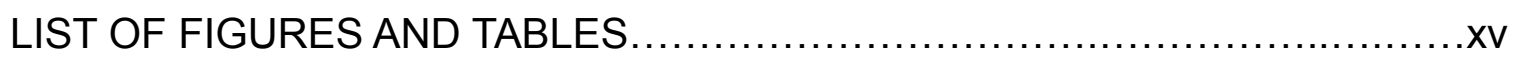

\section{CHAPTER}

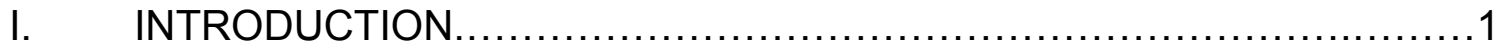

A. Background and rationale for this study ................................

1. Atypical antipsychotics: usage and side effects....................

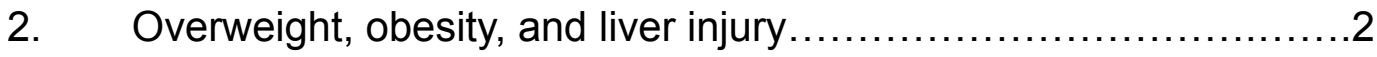

3. Olanzapine, obesity, and hepatic metabolism.......................

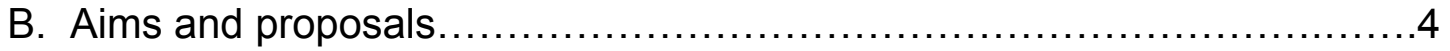

1. Does olanzapine directly alter hepatic energy metabolism? $\ldots \ldots \ldots \ldots . . .4$

2. Can olanzapine act as a "second hit" in fatty liver diseases?..........5

3. Can Nrf2 activation with sulforaphane protect against olanzapine-

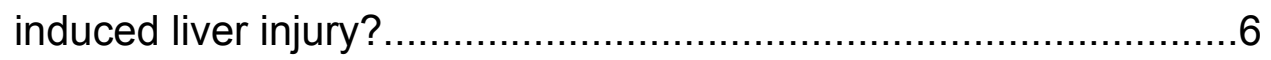

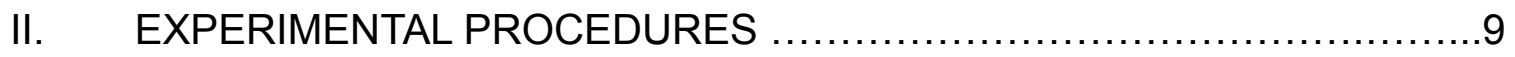




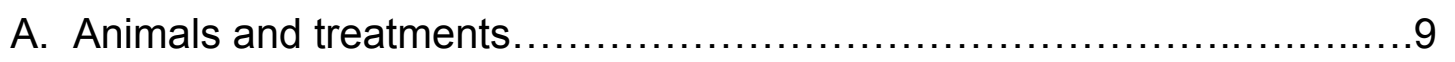

B. Chronic model of olanzapine exposure .................................. 10

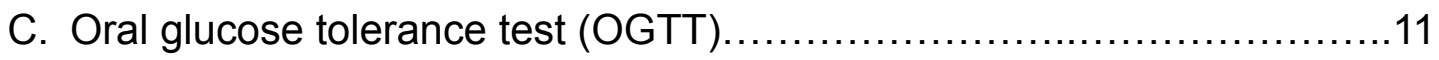

D. Biochemical analyses...................................................

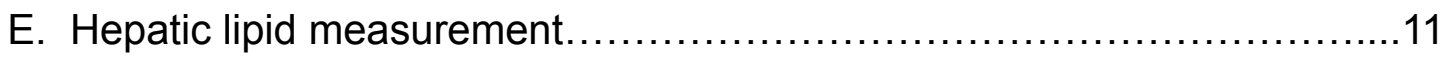

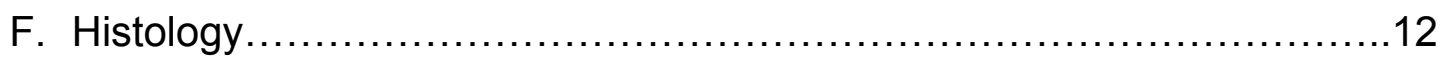

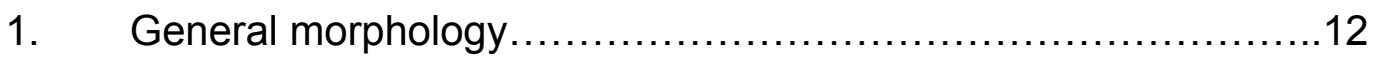

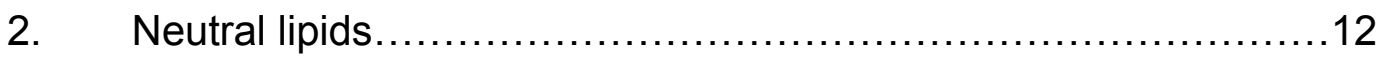

3. Glycogen and glycoprotein storage ............................12

4. $\quad$ Lipid peroxidation..................................................

5. Neutrophil accumulation.......................................13

6. Apoptosis.......................................................

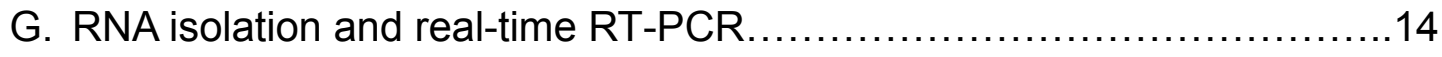

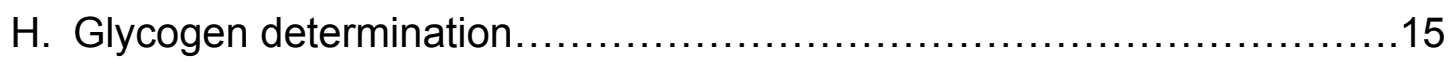

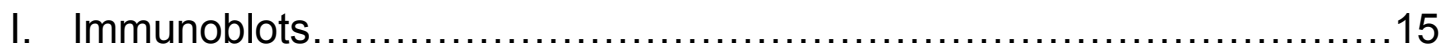

J. In vitro measurement of oxygen consumption and proton production rates

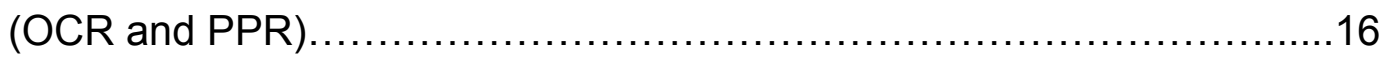

K. GC×GC-TOF MS and pathway analysis................................17

1. Metabolite sample preparation.................................17 


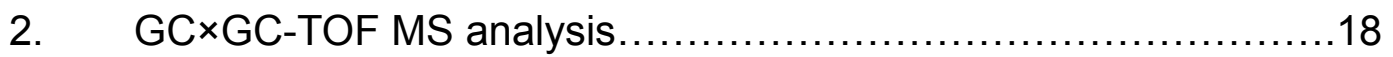

3. GC $\times$ GC-TOF MS data analysis ............................... 19

L. Sulforaphane administration........................................... 19

III. NEW MECHANISTIC INSIGHT INTO OLANZAPINE-INDUCED

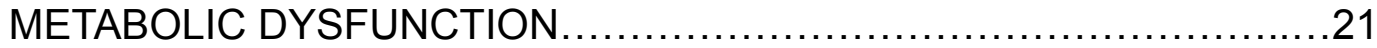

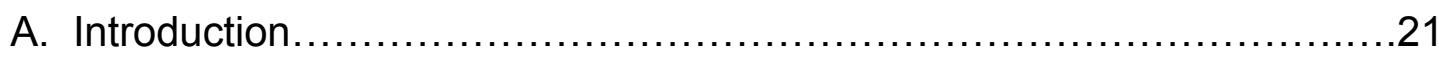

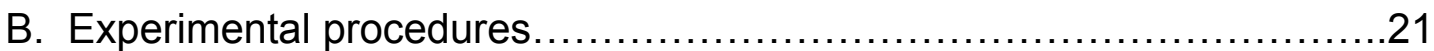

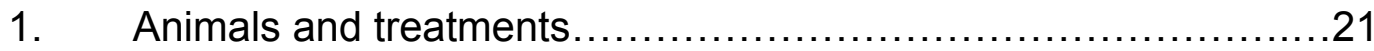

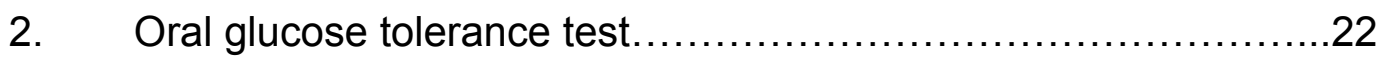

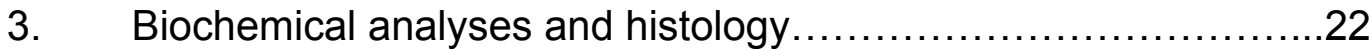

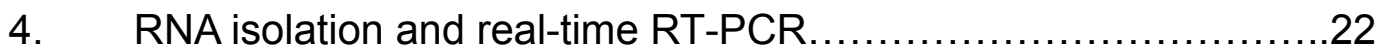

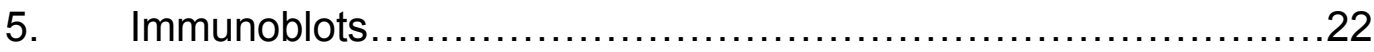

6. In vitro metabolic measurements .................................22

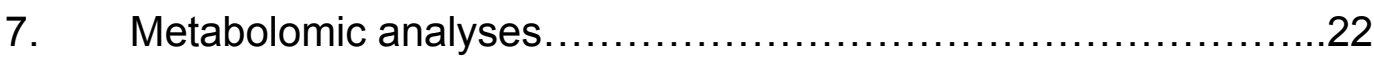

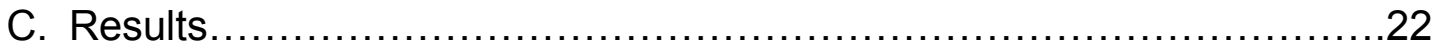

1. OLZ administration increases weight and adiposity ...............22

2. OLZ promotes hepatic lipid accumulation........................23

3. OLZ modifies expression of metabolism-regulating genes..........23

4. Effects of OLZ on mitochondrial respiration......................24 
5. Effects of OLZ on glucose and glycogen expenditure. .25

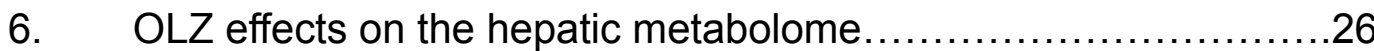

7. OLZ mediates signaling through mTOR and related pathways.....27

D. Discussion.

IV. HIGH-FAT DIET EXACERBATES OLANZAPINE-INDUCED LIVER

INJURY

A. Introduction

53

B. Experimental Procedures

1. Animals and treatments........................................... 53

2. Oral glucose tolerance test........................................

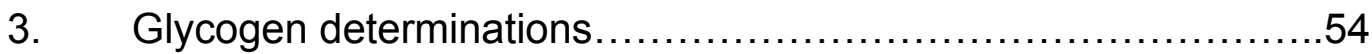

4. RNA isolation and real-time RT-PCR ...........................54

5. Biochemical analyses and histology .............................5

6. Lipid determinations.............................................. 54

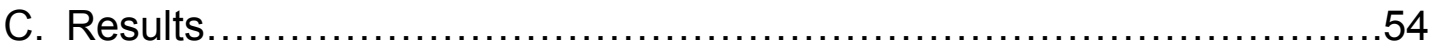

1. High-fat diet (HFD) increases food consumption and exacerbates

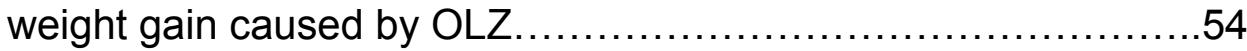

2. HFD aggravates OLZ-induced liver injury........................55

3. OLZ and HFD promote hepatic lipid storage ......................55 
4. Effects of OLZ and HFD on glucose metabolism..................56

5. HFD worsens OLZ-induced glycogen depletion..................57

6. Effects of HFD and OLZ on apoptosis and lipid peroxidation........57

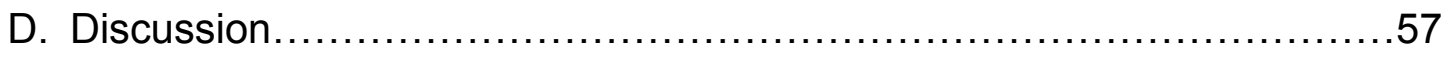

V. PROTECTION FROM OLANZAPINE-INDUCED LIVER INJURY

THROUGH ACTIVATION OF THE NRF2 PATHWAY .....................72

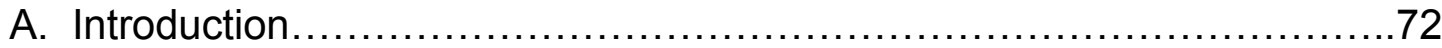

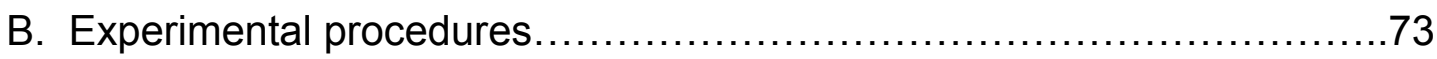

1. Animals and treatments..........................................

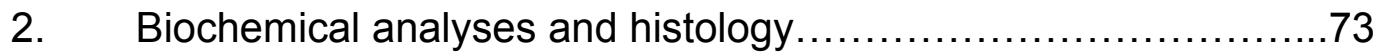

3. Glycogen determinations......................................73

4. Lipid determinations............................................

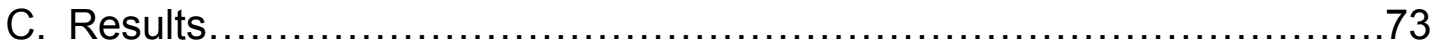

1. SFN prevents steatosis and liver injury caused by co-administration

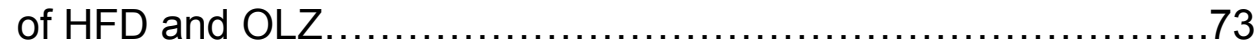

2. SFN improves lipid and carbohydrate metabolism in mice

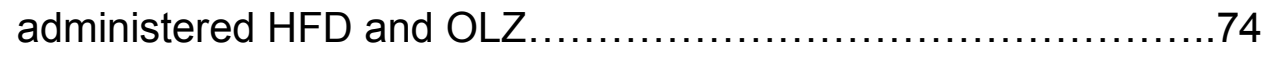

3. SFN does not affect apoptosis with HFD and OLZ, but greatly decreases lipid peroxidation...................................... 74 
D. Discussion.

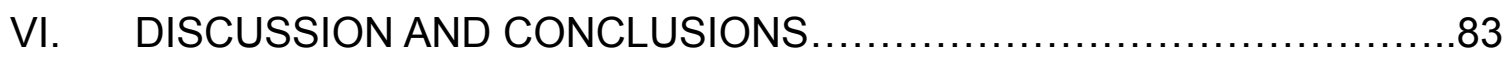

A. Biochemical pathways involved in NAFLD and/or OLZ toxicity and potential pharmacological interventions................................ 83

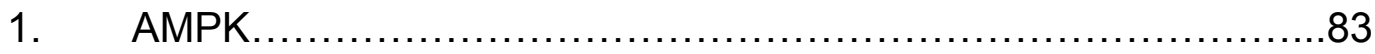

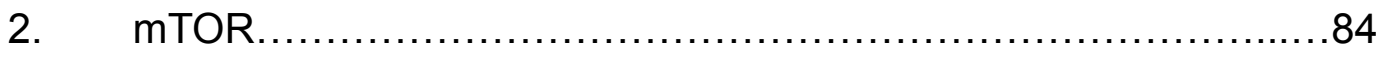

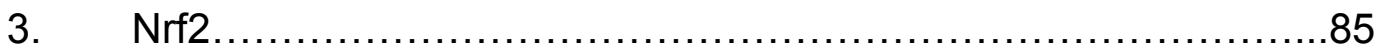

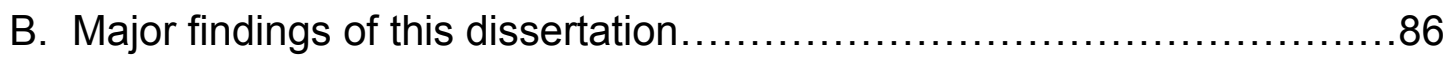

1. OLZ simultaneously activates AMPK and mTOR ...................86

2. OLZ modulates intracellular amino acid transport, which may affect energy homeostasis............................................. 88

3. HFD aggravates liver injury caused by OLZ .......................89

4. SFN counteracts oxidative stress caused by HFD and OLZ ........90

C. Strengths and weaknesses of this dissertation...........................91

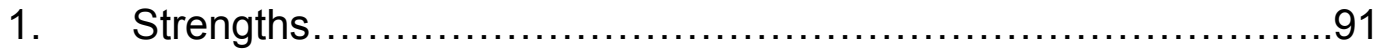

2. Weaknesses....................................................

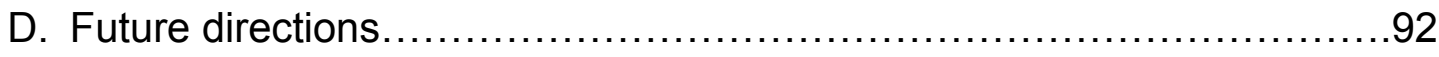

1. What are the effects of combined HFD and OLZ exposure on AMPK

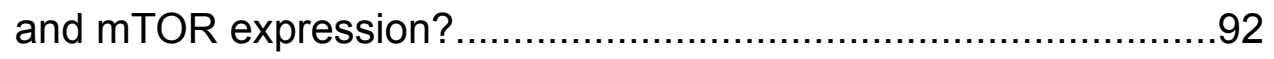


2. Does SFN influence absorption, distribution, metabolism, or excretion of OLZ?...

3. How does OLZ behave in other peripheral locations, such as

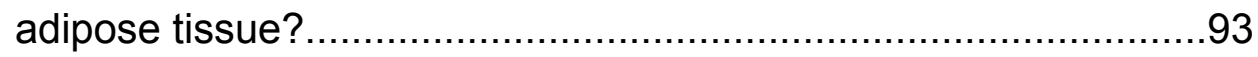

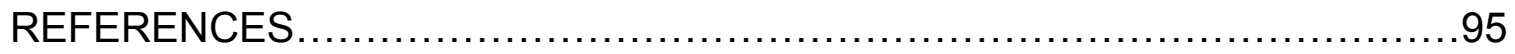

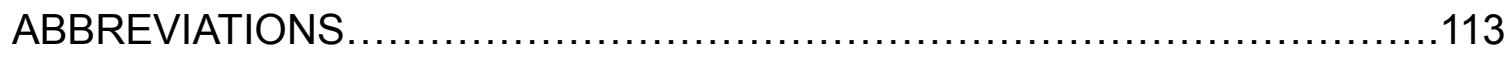

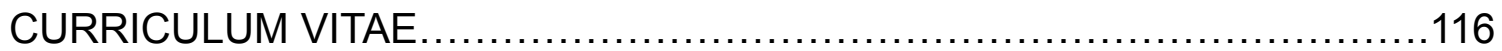




\section{LIST OF FIGURES AND TABLES}

TABLE

PAGE

3.1: Plasma parameters after 4 weeks of OLZ treatment......................30

\section{FIGURE}

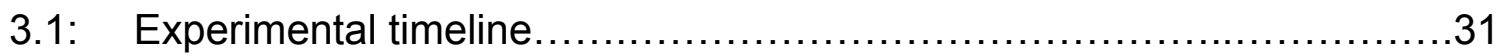

3.2: Effect of OLZ on body weight gain and adiposity ........................33

3.3: $\quad$ OLZ promotes hepatic lipid accumulation................................. 35

3.4: Effects of OLZ on hepatic gene expression............................37

3.5: Glucose vs. galactose media for immortalized cells.......................39

3.6: In vitro metabolism of OLZ-treated HepG2 cells.........................41

3.7: Effects of OLZ on glucose and glycogen expenditure....................43

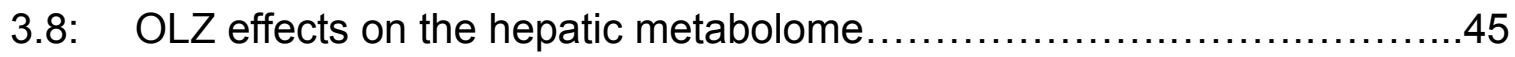

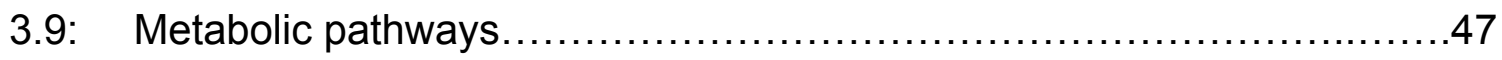

3.10: OLZ mediates signaling through mTOR and related pathways............49

3.11: Hypothesized mechanism of OLZ-induced metabolic dysregulation in

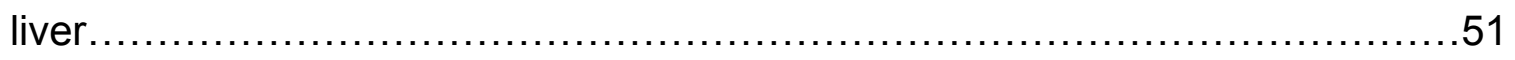




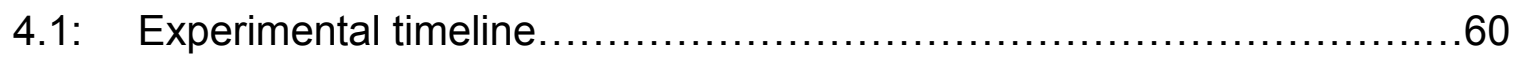

4.2: High-fat diet (HFD) increases food consumption and exacerbates weight

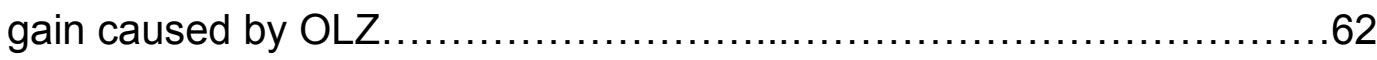

4.3: $\quad$ HFD aggravates OLZ-induced liver injury ..............................64

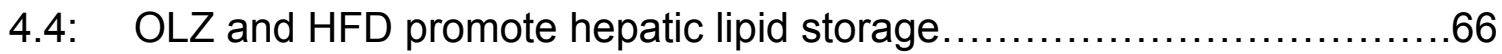

4.5: Effects of OLZ and HFD on glucose and glycogen metabolism...........68

4.6: Effects of OLZ on apoptosis and lipid peroxidation ......................70

5.1: Simplified model of Nrf2 induction by SFN ............................77

5.2: Influence of SFN on the metabolic outcomes of HFD and OLZ co-

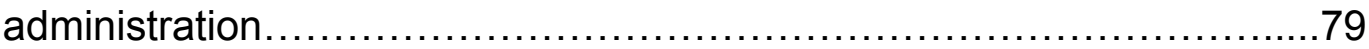

5.3: Histological indices of protection from HFD and OLZ by SFN.............81 


\section{CHAPTER I \\ INTRODUCTION}

\section{A. Background and rationale for this study}

\section{Atypical antipsychotics: usage and side effects}

Olanzapine $(O L Z)$ is one of the most effective drug options for managing the symptoms of schizophrenia and bipolar disorder. ${ }^{1,2}$ Much like earlier antipsychotic drugs (e.g., haloperidol), OLZ and other second generation antipsychotic medications bind antagonistically at dopamine receptors. ${ }^{3}$ Unlike the earlier antipsychotic medications, OLZ binds and antagonizes serotonin receptors, particularly the $5-\mathrm{HT}_{2 \mathrm{C}}$ receptor, and has a lower affinity for the $\mathrm{D}_{2}$ subclass of dopamine receptors. OLZ's different affinities for various neurotransmitter receptors may explain why it is less likely to cause tardive dyskinesia and movement-related side effects than the previous generation of drugs. ${ }^{4}$ Even among second generation antipsychotic drugs, OLZ is preferred for its ability to treat psychiatric symptoms, ${ }^{5,6}$ and in contrast to the chemicallyrelated clozapine, there is little or no risk of developing blood disorders. ${ }^{7}$ These attributes make OLZ a drug of choice to treat severe mental illness. Indeed, the lack of debilitating side effects has advanced the off-label use of the drug for

indications such as dementia and treatment-resistant anxiety disorders. ${ }^{8,9}$ 
Although OLZ does not share the severe toxicity of its first-generation predecessors, it does have side effects that limit its therapeutic potential. Numerous studies show that OLZ causes substantial and reproducible weight gain. ${ }^{10-12}$ Though exact figures vary, a meta-analysis conducted by Allison et al. found a mean weight gain of $4.0 \mathrm{~kg}$ after 10 weeks of OLZ administration. ${ }^{13}$ Weight gain with OLZ does not usually reach a rapid plateau, but rather continues gradually throughout the years of treatment. ${ }^{10}$ Prescribing information for Zyprexa, the brand-name formulation of OLZ, reports that the percent of adult patients with weight gain $>15 \mathrm{~kg}$ was $4.3 \%$ after 6 months of OLZ administration, $14.1 \%$ after 12 months, $17.9 \%$ after 24 months and $22.5 \%$ after 36 months. ${ }^{14,15}$ Side effects such as weight gain are additionally correlated with decreased treatment compliance. ${ }^{16,17}$ Overall, approximately $58 \%$ of patients requiring antipsychotic medication do not adhere to treatment plans, ${ }^{18}$ leading to relapse and increasing the need for costly inpatient psychiatric hospital stays. ${ }^{19,20}$

\section{Overweight, obesity, and liver injury}

Obesity is a major cause of preventable illness worldwide. ${ }^{21}$ In the United States obesity has reached epidemic proportions, with approximately $68.6 \%$ of Americans meeting the criteria for overweight (body mass index $\geq 25$ ) and $33.8 \%$ meeting the criteria for obesity (BMI $\geq 30$ ) according to the most recent National Health and Nutrition Examination Survey (NHANES III). ${ }^{22}$ Importantly, there is an upward trend in BMI in the United States: the prevalence of obesity has doubled since NHANES data were initially compiled in the $1960 \mathrm{~s}^{23}$

Among the myriad health complications associated with obesity (e.g., 
cardiovascular disease) is non-alcoholic fatty liver disease (NAFLD), which has become one of the leading causes of chronic liver disease worldwide. ${ }^{24}$ NAFLD is pathologically similar to alcoholic liver disease (ALD), but is distinguished by its development in the absence of significant alcohol consumption. ${ }^{25}$ Apart from obesity the risk factors for primary NAFLD include type II diabetes mellitus and dyslipidemia. Though its features are well-defined, NAFLD is not a single, uniform illness. Instead, it encompasses a spectrum of liver disease states, ranging from simple steatosis, to active inflammation (non-alcoholic steatohepatitis, or $\mathrm{NASH}$ ), to advanced fibrosis and cirrhosis, ${ }^{26}$ and ultimately hepatocellular carcinoma. ${ }^{27}$ Not all those with NAFLD are severely overweight; however, the majority of NAFLD patients do have metabolic risk factors such as obesity, hyperlipidemia, and/or type 2 diabetes mellitus. ${ }^{28}$ According to NHANES III data, an estimated 32.5 million people $-21.7 \%$ of the United States noninstitutionalized population between the ages of 20 and 74 - had some degree of hepatic steatosis. Of these individuals, 28.8 million, or $90 \%$, were considered by NHANES to have NAFLD. ${ }^{29}$

\section{Olanzapine, obesity, and hepatic metabolism}

The side effects of OLZ are particularly relevant at this time, with obesity increasing at an alarming rate in the US and other industrialized countries. ${ }^{30}$ Moreover, even within an increasingly overweight general population, obesity disproportionately affects individuals with severe mental illnesses. ${ }^{31,32}$ When a cohort of randomly selected psychiatric outpatients was compared to a gender-, race- and age-matched group from NHANES III, the prevalence of obesity in the 
outpatient psychiatric population was nearly double that of the NHANES group. ${ }^{33}$ Weight gain is not the only major metabolic effect of antipsychotic administration: OLZ has also been associated with liver injury, ${ }^{34}$ dyslipidemia, ${ }^{35}$ and insulin resistance..$^{11,36}$ These effects, as mentioned previously, increase overall patient mortality ${ }^{37}$ and strongly negatively influence patient treatment compliance. It is therefore necessary to develop strategies that prevent, minimize, or reverse the adverse metabolic effects that occur during OLZ treatment, especially for a population already at risk for obesity and its sequelae.

The purpose of the work described in the chapters that follow was to explore the effects of OLZ on indices of carbohydrate and lipid metabolism. Ultimately, the goal was to determine relevant mechanisms in the development, progression and treatment of OLZ-induced liver injury. The following hypotheses are central to this dissertation:

\section{B. Aims and proposals}

\section{Does olanzapine directly alter hepatic energy metabolism?}

Hepatic metabolism is paramount to this study for several reasons. First, the liver is indispensable to whole-body glycemic control and lipid regulation. The liver acts as a site for glucose uptake and gluconeogenesis, and provides a large store of glycogen for the maintenance of blood glucose during fasting. ${ }^{38}$ Despite its somewhat small size in proportion to the rest of the body, the liver accounts for $\sim 20 \%$ of the body's resting energy expenditure. ${ }^{39}$ Secondly, regardless of whether it is administered orally or parenterally, OLZ reaches high concentrations in liver, far surpassing levels in kidney, plasma and brain. ${ }^{40}$ 
Finally, the liver's role in the development of metabolic side effects may be more important than previously thought. The beneficial effects of $O L Z$ are assumed to be mediated at the level of the CNS. Not surprisingly, most studies into the metabolic side effects of OLZ have also focused on the CNS. Although the CNS clearly plays a key role in regulating food consumption, obesity, dyslipidemia and diabetes, ${ }^{41-43}$ recent studies have suggested that the hepatic effects of OLZ contribute to the drug's metabolic disturbances. ${ }^{44}$ Interestingly, the changes induced by OLZ administration in carbohydrate and lipid metabolism may in fact precede weight gain, which suggests a potential direct effect of the drug on these pathways. ${ }^{36,45}$ The purpose of the experiments described in Chapter III was to determine what direct effects OLZ administration has on the liver, in the absence of underlying illness or additional treatment.

\section{Can OLZ act as a second "hit" in fatty liver diseases?}

As described in the previous section, NAFLD is a spectrum of liver diseases spanning from the relatively benign (steatosis) to the most severe (liver cancer) ${ }^{27}$ The prevalence of simple steatosis in individuals at risk for NAFLD can be very high; for example, the prevalence in individuals with a body mass index (BMI) of 35 or greater has been reported to be $90 \% .{ }^{46}$ In contrast, the prevalence of $\mathrm{NASH}$ is much lower in this population $(\sim 40 \%) .{ }^{46}$ These factors emphasize that the risk for developing more severe stages of NAFLD (i.e., NASH and beyond) is not based solely on obesity, but is rather mitigated by genetic or environmental factors - that is, disease progression is governed by the '2-hit' paradigm. The paradigm proposes that physiological and/or biochemical 
changes to liver that are pathologically inert can aggravate the hepatotoxic response caused by a second agent. ${ }^{47,48}$ It is possible that OLZ treatment could be such a previously unidentified agent in the progression of NAFLD. The purpose of the experiments described in Chapter IV was to assess the impact of OLZ on liver pathology in mice already predisposed to obesity and liver injury.

\section{Can Nrf2 activation with sulforaphane protect against OLZ-induced} liver injury?

The formation of electrophilic and oxidative molecules is an invariable consequence of normal metabolism. ${ }^{49}$ Reactive metabolites are generally neutralized and excreted, though they may also serve as intermediates in cell signaling. ${ }^{50,51}$ The oxidant-antioxidant equilibrium is, in most cases, wellregulated by a series of enzymes specifically evolved for this purpose. However, an excess of oxidant species and/or a lack of antioxidant species will sometimes develop, and it is this state of imbalance that is known as oxidative stress. ${ }^{52}$

Oxidative stress is not defined as a disease, though it is widely hypothesized to be a common mechanism in the initiation or progression of many diseases. Manifestations of oxidative stress such as lipid peroxidation are repeatedly observed in conjunction with metabolic diseases. ${ }^{53-56}$ High levels of fatty acids, cholesterol, and glucose produce reactive oxygen species, ${ }^{57-59}$ and may therefore be crucial to the progression from obesity to more serious illnesses such as NASH. ${ }^{60,61}$ It may also follow that the metabolic side effects of OLZ can be prevented by antioxidants. Though the exact involvement of antioxidant pathways in antipsychotic action is a matter of debate, OLZ was 
recently reported to raise levels of malondialdehyde, a common biomarker for oxidative stress. $^{62}$

Past research has largely used direct antioxidants (e.g., vitamin C) to prevent drug-induced oxidative stress. This approach has been more consistently successful in vitro than in whole organisms. At worst, direct-acting treatments actually induce disturbances in cellular redox status. ${ }^{63-65} \mathrm{~A}$ metaanalysis of clinical trials using vitamin $\mathrm{E}$ found that in high doses, vitamin $\mathrm{E}$ significantly increases all-cause mortality. ${ }^{66}$ The toxicity of supplements such as vitamin E may result from their dual nature: at high doses, many antioxidants act as pro-oxidants. ${ }^{67,68}$ In contrast, indirect agents such as sulforaphane (SFN) have neither reducing nor oxidizing capability. ${ }^{69,70}$ SFN instead works by inducing Nrf2, thereby enhancing the cell's own reducing capability and supporting the function of endogenous detoxifying enzymes. ${ }^{71}$

There is already some evidence that alleviating oxidative stress can alleviate the side effects of OLZ. Although high-dose vitamin E did not improve insulin resistance in OLZ-medicated adults in a small clinical trial conducted by Salmasi et al., ${ }^{72}$ Shertzer et al. protected mice against OLZ-mediated metabolic toxicity with tetrahydroindenoindole, an antioxidant, ${ }^{73}$ and acetaminophen, which functioned as an antioxidant at the dose in which it was given. ${ }^{74}$ Similarly, a study by Mas et al. used SFN to prevent oxidative stress in neuroblastoma cells exposed to the second-generation antipsychotic drug risperidone. ${ }^{75}$ It remains possible that oxidative stress is crucial to metabolic side effect development, but there are few studies similar to those of Shertzer or Mas. The purpose of the 
experiments described in Chapter V was to test Nrf2 induction (via SFN) as a strategy against obesity- and OLZ-induced dysregulation of metabolism, particularly in the liver. 


\section{CHAPTER II \\ EXPERIMENTAL PROCEDURES}

\section{A. Animals and treatments}

Female C57BL/6J mice (8 weeks old) were purchased from The Jackson Laboratory (Bar Harbor, ME). Mice were housed in a pathogen-free barrier facility accredited by the Association for Assessment and Accreditation of Laboratory Animal Care. All procedures were approved by the University of Louisville Institutional Animal Care and Use Committee.

Food and tap water were allowed ad libitum throughout the experiment, except for a single 6 hour fast before glucose tolerance testing (see sections below for more details). One week prior to the initiation of OLZ, animals were switched from laboratory chow to standardized diets (Harlan Laboratories, Madison, $\mathrm{WI}$ ) to avoid any batch-to-batch variability in the food content. ${ }^{76}$ In studies designed to test the effects of OLZ alone, pellets of TD.08485 (Low-Fat Control Diet; $13 \%$ calories from fat) were supplied fresh twice per week. In studies designed to test the interaction between OLZ and high-fat diet, pellets of either TD.08485 or TD.88137 (Adjusted Calories Diet; 42\% calories from fat) were supplied fresh twice per week. Food consumption was estimated by 
subtracting the weight of the pellets remaining at the end of each semi-weekly feeding period.

\section{B. Chronic model of olanzapine exposure}

Olanzapine ( $8 \mathrm{mg} / \mathrm{kg} / \mathrm{d})$ or vehicle (saline) was given s.c. for 28 days via surgically implanted osmotic minipumps (Alzet, Cupertino, CA). This dose of OLZ was previously determined by positron emission tomography scan to achieve a therapeutically-relevant level of dopamine $\left(D_{2}\right)$ receptor occupancy in mice. ${ }^{77}$ The s.c. route of administration was chosen to maintain a steady dosage of OLZ, and because parenteral and oral formulations of OLZ have already demonstrated comparable efficacy in vivo. ${ }^{78}$ To avoid concerns of OLZ degradation, pumps were replaced after two weeks. ${ }^{79}$ Individual mouse weights were recorded on a weekly basis. After 28 days, the mice were anesthetized with ketamine/xylazine (100/15 mg/kg i.p.) and minipumps were removed and weighed. Body composition was assessed by dual-energy $\mathrm{x}$-ray absorptiometry (DEXA Scan) ${ }^{80}$ using a Lunar PIXImus densitometer (Lunar Corp., Madison, WI). Blood was collected from the vena cava just prior to sacrifice by exsanguination, and citrated plasma was stored at $-80{ }^{\circ} \mathrm{C}$ for later analysis. Portions of liver tissue were frozen immediately in liquid nitrogen, fixed in $10 \%$ neutral buffered formalin, or frozen-fixed in cold embedding medium (Tissue-Tek OCT compound, Sakura Finetek, Torrance, CA) for subsequent sectioning and mounting on microscope slides. Blood, liver, and gonadal fat pads were collected for later analysis. 


\section{Oral glucose tolerance test (OGTT)}

Glucose tolerance was evaluated at day 25 of OLZ exposure using an OGTT method optimized by Andrikopoulos et al. ${ }^{81}$ Mice were transferred to cages that had been cleared of food and bedding and fasted for 6 hrs. Blood was sampled from the tail vein immediately after fasting, and then $15,30,60,90$ and $120 \mathrm{~min}$. after oral administration of $2 \mathrm{mg} / \mathrm{kg} \mathrm{D-(+)-glucose} \mathrm{(Sigma,} \mathrm{St.} \mathrm{Louis,}$ MO) solution. Glucose concentrations were measured using an Accu-Chek® Aviva Plus glucometer and test strips (Roche Diagnostics Corp., Indianapolis, IN).

\section{Biochemical analyses}

Plasma levels of alanine aminotransferase (ALT), aspartate aminotransferase (AST), total cholesterol, HDL, LDL, VLDL, triglycerides (TG), and glucose were determined by the Piccolo $®$ Lipid Panel Plus Reagent Disc, used with the Piccolo xpress ${ }^{\mathrm{TM}}$ Chemistry Analyzer (Abaxis, Inc., Union City, $\mathrm{CA}$ ), according to the manufacturer's instructions.

\section{E. Hepatic lipid measurement}

Hepatic lipids (TG and NEFA - non-esterified fatty acids) were extracted as described by Bligh and Dyer. ${ }^{82}$ Briefly, mouse livers were pulverized in a mortar filled with liquid nitrogen. Lipids were extracted from the pulverized tissue by separation in a 1:2 solution of methanol and chloroform, then dried under compressed nitrogen gas and resuspended in $5 \%$ fat-free bovine serum albumin 
(Sigma, St Louis, MO). Results were normalized to wet weight of extracted tissue.

\section{F. Histology}

\section{General morphology}

Hematoxylin and eosin (H\&E) staining was used to assess overall hepatic structure. Paraffin-embedded sections of liver $(5 \mu \mathrm{m})$ were deparaffinized and rehydrated, stained with hematoxylin for 45 seconds, washed, and counterstained with eosin for 3 minutes. Stained sections were then mounted with Permount (Fisher Scientific, Fair Lawn, NJ) and glass coverslips.

\section{Neutral lipids}

For detection of neutral lipids in tissue, $10 \mu \mathrm{m}$ sections of frozen-fixed liver embedded in OCT Compound were cut and stained with Oil Red O (Sigma Chemical Co., St. Louis, MO) for 10 min., washed, and counterstained with hematoxylin for 45 seconds before mounting with Crystal Mount ${ }^{\mathrm{TM}}$ (Sigma, St. Louis, MO). The extent of oil Red O staining was defined as the percent of the

field area within the default color range determined by image analysis software. ${ }^{83}$

\section{Glycogen and glycoprotein storage}

Carbohydrate storage was visualized with Periodic acid-Schiff (PAS) reagent staining. Paraffin sections were oxidized in $0.5 \%$ periodic acid solution for 5 min., washed, placed in Schiff reagent (Sigma) for 15 min., washed, and counter-stained with hematoxylin for $90 \mathrm{sec}$. Similar to Oil Red O staining, the 
extent of PAS staining was defined as the percent of the microscope field area within the default color range determined by image analysis software. ${ }^{83}$

\section{Lipid peroxidation}

Adducts of 4-hydroxynonenal, an $\alpha, \beta$-unsaturated hydroxyalkenal produced by lipid peroxidation, were detected with a modified version of

immunohistochemical techniques described by Arteel et al. ${ }^{83,84}$ Specifically, tissue sections were stained using a rabbit polyclonal 4-HNE antibody (Alpha Diagnostic, San Antonio, Texas) and a kit based on a routine biotin-streptavidinperoxidase staining technique (VECTASTAIN ABC kit, Vector Laboratories, Burlingame, CA). Once the antibody-biotin-peroxidase complex was formed, diaminobenzidine (ImmPACT ${ }^{\mathrm{TM}}$ DAB peroxidase substrate, Vector Laboratories) was added as the peroxidase substrate. After the immunostaining procedure, tissue sections were counterstained with hematoxylin and mounted. Staining was quantitated as the percentage of brown labeling (i.e., DAB) of the field area minus the area of the acellular spaces.

\section{Neutrophil accumulation}

For measurement of hepatic neutrophil infiltration, the naphthol AS-D chloroacetate esterase (CAE) kit (Sigma, St. Louis, MO) was used according to the manufacturer's instructions. CAE-positive cells were quantitated by counting (positive cells per 1000 hepatocytes) in randomly selected fields. ${ }^{85}$

\section{Apoptosis}

Apoptosis was assessed by fluorescent TdT-mediated dUTP nick-end 
labeling (TUNEL) employing a commercially available kit (ApopTag® Red In Situ Apoptosis Detection Kit, CHEMICON International, Temecula, CA). Slides were mounted with VECTASHIELD mounting medium containing DAPI (Vector Laboratories) and glass coverslips. Apoptotic cells were counted in the same manner as CAE-positive cells (see Neutrophil accumulation above).

A MetaMorph ${ }^{\mathrm{TM}}$ image analysis system (Universal Imaging Corporation, Downingtown, PA) equipped with a Nikon microscope (Nikon, Melville, NY) was used to capture and analyze the results of the staining procedures described above. Positive staining was not detected when tissues were processed without primary antibody, indicating that nonspecific binding of secondary antibody did not occur under these conditions.

\section{G. RNA Isolation and Real-Time RT-PCR}

Message levels of selected genes were detected by real-time reversetranscriptase PCR. Total RNA was extracted from tissue samples by a guanidium thiocyanate-based method (Tel-Test, Austin, TX). RNA concentrations were determined spectrophotometrically, and $1 \mu \mathrm{g}$ total RNA was reverse transcribed using an MMLV reverse transcriptase kit (Quanta Biosciences, Gaithersburg, MD). The cDNA was added to a mixture containing premade primers and probes (Applied Biosystems, Foster City, CA) and a ready-to-use reaction buffer (Quanta Biosciences). Amplification reactions were performed using ABI StepOne Plus Software (Applied Biosystems). The comparative CT method was used to determine fold differences between samples. The comparative CT method 
determines the amount of target, normalized to an endogenous reference ( $\beta$ actin) and relative to a calibrator (2- $\Delta \Delta \mathrm{Ct})$.

\section{H. Glycogen Determination}

To determine hepatic glycogen content, mouse livers $(50-100 \mathrm{mg})$ were weighed and placed immediately in $30 \% \mathrm{KOH}(500 \mu \mathrm{l})$, digested at $100^{\circ} \mathrm{C}$ for 20 min, then cooled to room temperature. To the cooled digest, 95\% EtOH (625 $\mu$ l) was added and allowed to stand overnight at room temperature. The samples were then centrifuged at $16000 \times g\left(4^{\circ} \mathrm{C}\right)$ for $15 \mathrm{~min}$. The resultant pellet was resuspended in $1 \mathrm{ml}$ water. Hepatic glycogen content was then determined with anthrone reagent as described by Seifter et al. ${ }^{86}$ and modified for a 96 -well plate by Leyva et al. ${ }^{87}$ Glycogen content was calculated from glucose stand curve and reported at $\mu \mathrm{g} / \mathrm{g}$ tissue.

\section{Immunoblots}

Frozen liver samples were homogenized (Polytron Kinematica, Lucerne, Switzerland) in RIPA buffer (20 mM Tris pH 7.4, 150mM NaCl, 1mM EDTA, 1 mM EGTA, $1 \mathrm{mM} \beta$-glycerophosphate, $1 \mathrm{mM} \mathrm{Na3VO}$, and 1\% w/w Triton X100) containing protease, tyrosine phosphatase, and serine/threonine phosphatase inhibitor cocktails (Sigma, St. Louis, MO). Lysates were agitated with an ultrasonic probe and subsequently centrifuged for 5 min. at $16,000 \times g$. Protein concentration of the supernatants was determined with the Bio-Rad DC Protein Assay (Bio-Rad, Hercules, CA, USA); $100 \mu \mathrm{g}$ of total protein were mixed with $4 \times$ sample loading buffer $(250 \mathrm{mM}$ Tris $\mathrm{pH} 7.4,10 \%$ SDS, $20 \% 2-$ mercaptoethanol, $40 \%$ glycerol and $0.01 \% \mathrm{w} / \mathrm{v}$ bromophenol blue) and incubated 
at $95^{\circ} \mathrm{C}$ for $5 \mathrm{~min}$. Samples were loaded onto SDS-polyacrylamide gels (BioRad Laboratories, Hercules, CA), followed by electrophoresis and Western blotting onto PVDF membranes (Hybond P, Amersham Biosciences, Piscataway, NJ, USA). Antibodies against p-AMPKa, AMPKa, p-Akt, Akt, p-mTOR, mTOR, pp70S6K, p-4EBP1 (Cell Signaling, Danvers, MA, USA) and p70S6K and 4EBP1 (Bethyl Laboratories, Montgomery, TX) were used at the dilutions recommended by the suppliers. Horseradish peroxidase-coupled secondary antibodies and chemiluminescence detection reagents were from Pierce (Rockford, IL, USA). The signals were detected employing Classic Blue ${ }^{\mathrm{TM}}$ autoradiography film BX (MIDSCl, St. Louis, MO). Densitometric quantitation was performed with UNSCAN IT analysis software (Silk Scientific, Orem, UT).

\section{J. In vitro measurement of oxygen consumption and proton production rates (OCR and PPR)}

Bioenergetic measurements were made using an XF96 Extracellular Flux Analyzer (Seahorse Biosciences, Billerica, MA). HepG2 cells (American Type Culture Collection, Manassas, VA) were plated at 10,000 cells per well and grown for 24 hrs. in DMEM (Gibco, Carlsbad, CA) containing either glucose (25 $\mathrm{mM}$ ) or galactose $(10 \mathrm{mM})$ and $6 \mathrm{mM}$ pyruvate. (Immortalized cells preferentially use glycolysis to meet energy demands, even in the presence of adequate oxygen. ${ }^{88}$ Anaerobic glycolysis of galactose yields no net ATP, however, forcing cells to rely on oxidative phosphorylation for survival.) Cells were then treated with graded concentrations of olanzapine from $0-25 \mu \mathrm{M}$. One hour prior to the commencement of measurements the media was changed to unbuffered DMEM 
(Seahorse Biosciences) containing the same concentrations of sugars, pyruvate or olanzapine. The XF96 Extracellular Flux analyzer measures OCR (oxygen consumption rate) and PPR (proton production rate) by creating a small transient chamber in specialized plates. Seeding density was optimized under the cell culture conditions described to ensure linear oxygen consumption and adequate re-equilibration during the re-equilibration phases. Coupled and uncoupled OCR and PPR measurements were made by addition of oligomycin $(1 \mu \mathrm{g} / \mathrm{ml})$ and FCCP $(1 \mu \mathrm{M})$, respectively, through sequential injections from ports in the Seahorse Flux Pak cartridges.

\section{K. GCXGC-TOF MS and Pathway Analysis}

\section{Metabolite sample preparation}

A sample of liver tissue was weighed and homogenized for 2 min after adding water at a ratio of $100 \mathrm{mg}$ liver tissue $/ \mathrm{mL}$ water. The homogenized sample was then stored at $-80{ }^{\circ} \mathrm{C}$ until use. A $100 \mu \mathrm{L}$ aliquot of the homogenized liver sample and $400 \mu \mathrm{L}$ ice cold methanol were mixed and vortexed for $2 \mathrm{~min}$, then incubated for 10 min on ice followed by another 2 min vortex. The mixtures were centrifuged at $4{ }^{\circ} \mathrm{C}$ for $10 \mathrm{~min}$ at $15000 \mathrm{rpm} .400 \mu \mathrm{L}$ of the supernatant was aspirated into a micro centrifuge tube and dried by SpeedVac overnight. The extracted metabolites were then dissolved in $40 \mu \mathrm{L}$ acetonitrile. After adding 40 $\mu \mathrm{L} N$-(tert-butyldimethylsilyl)-N-methyltrifluoroacetamide (MTBSTFA) mixed with $1 \%$ tert-butyldimethylchlorosilane (TBDMSCI), the mixture was sonicated for 3 hours followed by overnight derivatization at room temperature. The samples 
were then transferred to $\mathrm{GC}$ vials for analysis. The derivatization was performed just before GC×GC-TOF MS analysis.

\section{GCXGC -TOF MS analysis}

The LECO Pegasus 4D GC×GC -TOF MS instrument was equipped with an Agilent 6890 gas chromatograph and a Gerstel MPS2 auto-sampler (GERSTEL Inc., Linthicum, MD), featuring a LECO two-stage cryogenic modulator and secondary oven. The primary column was a $60 \mathrm{~m} \times 0.25 \mathrm{~mm} 1 \mathrm{dc}$ $\times 0.25 \mu \mathrm{m} 1 \mathrm{df}$, DB-5ms GC capillary column (phenyl arylene polymer virtually equivalent to a (5\%-phenyl)-methylpolysiloxane). A second GC column of $1 \mathrm{~m} \times$ $0.25 \mathrm{~mm} \mathrm{1dc} \times 0.25 \mu \mathrm{m} 2 \mathrm{df}, \mathrm{DB} 17 \mathrm{~ms}$ ((50\%-phenyl)-methylpolysiloxane) was placed inside the secondary GC oven after the thermal modulator. Both columns were obtained from Agilent Technologies (Agilent Technologies J\&W, Santa Clara, CA). The helium carrier gas $(99.999 \%$ purity) flow rate was set to 2.0 $\mathrm{mL} / \mathrm{min}$ at a corrected constant flow via pressure ramps. The inlet temperature was set at $280{ }^{\circ} \mathrm{C}$. The primary column temperature was programmed with an initial temperature of $60^{\circ} \mathrm{C}$ for $0.5 \mathrm{~min}$ and then ramped at $5^{\circ} \mathrm{C} / \mathrm{min}$ to $270{ }^{\circ} \mathrm{C}$ and kept for $15 \mathrm{~min}$. The secondary column temperature program was set to an initial temperature of $70^{\circ} \mathrm{C}$ for $0.5 \mathrm{~min}$ and then also ramped at the same temperature gradient employed in the first column to $280{ }^{\circ} \mathrm{C}$ accordingly. The thermal modulator was set to $+15^{\circ} \mathrm{C}$ relative to the primary oven, and a modulation time of $P M=2 \mathrm{~s}$ was used. The mass range was set as $29-800 \mathrm{~m} / \mathrm{z}$ with an acquisition rate of 200 mass spectra per second. The ion source chamber was set at $230{ }^{\circ} \mathrm{C}$ with the transfer line temperature set to $280{ }^{\circ} \mathrm{C}$, and 
the detector voltage was $1450 \mathrm{~V}$ with electron energy of $70 \mathrm{eV}$. The acceleration voltage was turned on after a solvent delay of $674 \mathrm{~s}$. The split ratio was set at $25: 1$.

\section{GCXGC-TOF MS data analysis}

The GC×GC-TOF MS data were processed using LECO's instrument control software ChromaTOF for peak picking and tentative metabolite identification, followed by retention index matching, peak merging, peak list

alignment, normalization, and statistical significance test using MetPP software. ${ }^{89}$ For metabolite identification using ChromaTOF, each chromatographic peak was tentatively assigned to a metabolite if its experimental mass spectrum and a database spectrum have a spectral similarity score no less than 600 . The retention index matching in MetPP was performed using the iMatch method with the $p$-value threshold set as $p \leq 0.001 .^{90}$ The pairwise two-tail t-test was used to determine whether a metabolite has a significance abundance difference between sample groups by setting the threshold of false discovery rate $q \leq 0.3$.

\section{Sulforaphane administration}

Sulforaphane (SFN), a known Nrf2 inducer, was investigated as a possible preventative measure against weight gain and oxidative injury. In the experiments discussed in Chapter V, mice received SFN diluted in water (90 mg/kg/d p.o.; LKT Laboratories, St. Paul, MN) alongside chronic OLZ treatment and HFD feeding. SFN treatment began one week prior to OLZ administration (see Chapter II, section B, Chronic model of olanzapine exposure) and continued 
until the day of sacrifice. Mice not receiving sulforaphane received equivalent volumes of water as a treatment control. 


\section{CHAPTER III \\ NEW MECHANISTIC INSIGHT INTO OLANZAPINE-INDUCED METABOLIC DYSFUNCTION}

\section{A. Introduction}

The atypical antipsychotic olanzapine is a first-line treatment for psychosis and mood disorders. The major side effects of this centrally-acting drug occur in the peripheral tissues: weight gain, hepatic steatosis, and glucose intolerance. The exact mechanism or mechanisms leading to side effect development with OLZ are unknown. The purpose of the following experiments was to reveal the biochemical pathways involved in OLZ metabolism and toxicity, primarily in liver.

\section{B. Experimental procedures}

\section{Animals and treatments}

All mice in this experiment were fed purified TD.08485 diet starting one week prior to administration of OLZ or vehicle. OLZ administration and sacrifice procedures are described in Chapter II. See also: Timeline, Figure 3.1, panel A.

\section{Oral glucose tolerance test}

Glucose tolerance testing was performed as described in Chapter II. 


\section{Biochemical analyses and histology}

ALT, AST, cholesterol, TG and glucose were determined in plasma after

four weeks of OLZ administration according to the methods detailed in Chapter II. Liver sections were stained with H\&E, Oil Red O, and Periodic acid-Schiff reagent as described in Chapter II.

\section{RNA isolation and real-time RT-PCR}

See detailed methods in Chapter II.

\section{Immunoblots}

Immunoblotting was used to assess the phosphorylation status of mTOR, AMPK, 4EBP1, p70S6K and Akt, as described in Chapter II.

\section{In vitro metabolic measurements}

HepG2 cells were exposed to varying concentrations of $O L Z$ and measured with an extracellular flux analyzer as described in Chapter II.

\section{Metabolomic analyses}

GC $\times$ GC-TOF MS was performed and the results were analyzed as previously described in Chapter II.

\section{Results}

\section{OLZ administration increases weight and adiposity}

All animals gained weight during the course of the study and there was no mortality in any group. OLZ administration did not significantly increase food consumption compared to animals administered vehicle ("control"). Although 
food consumption measurements for each group were nearly identical, OLZ administration significantly increased body weight by $\sim 40 \%$ over controls (Figure

3.2, panel A), similar to what has been observed previously by other groups. ${ }^{91}$ An increase in body fat percentage and gonadal fat pad mass accompanied the weight gain (Figure 3.2, panel B) in OLZ-exposed animals.

\section{OLZ promotes hepatic lipid accumulation}

Obesity and/or metabolic syndrome commonly cause lipids to accumulate in the liver (i.e., steatosis). ${ }^{61}$ As OLZ administration increased body weight and total body fat (Figure 3.2), the effect of OLZ on hepatic lipid accumulation was determined. A crude index of hepatic lipid accumulation is liver size. OLZ increased liver weight as indicated by the elevation in liver weight to body weight ratio (Figure 3.3, panel C). Four weeks of OLZ treatment also increased hepatic fat, as indicated by the presence of macro- and micro-vesicular lipid droplets in hematoxylin and eosin-stained tissue (Figure 3.3, panel A, top). This effect of OLZ on hepatic lipid accumulation was confirmed by Oil Red O staining (panel A, bottom). OLZ administration significantly elevated hepatic triglyceride (TG) content $\sim 2$-fold (Figure 3.3, panel B); interestingly, this increase in TG was not coupled with a concomitant increase in hepatic non-esterified fatty acids (NEFA). This increase in hepatic lipids was paralleled by a significant increase in plasma triglycerides and VLDL (Table 3.1). These effects of OLZ were also accompanied by liver injury, as indicated by significant elevations of plasma ALT ( 2-fold) and AST ( 1.5 fold; Table 3.1).

\section{OLZ modifies expression of metabolism-regulating genes}


Hepatic steatosis is often mediated via direct alterations in the expression of genes key to lipid and carbohydrate metabolism. To explore the effects of OLZ on hepatic energy metabolism, mRNA expression of genes that are key in regulating the synthesis and catabolism of carbohydrates and lipids was examined by qRT-PCR (Figure 3.4). Four weeks of OLZ administration significantly downregulated the expression of a number of genes involved in lipid biosynthesis, including sterol regulatory element-binding protein 1 (Srebf1), fatty acid synthase (Fasn), and ATP citrate lyase (Acly). Similarly, OLZ treatment upregulated expression of glycogen synthase kinase-3 $\beta$ (Gsk3b), a protein that suppresses glycogen synthesis. These changes in expression of anabolismregulating genes were accompanied by a significant $(2.5$-fold) increase in expression of glucokinase (Gck), a rate-limiting enzyme in glycolysis. Expression of carnitine palmitoyltransferase 1a (Cpt1a), which encodes the rate-limiting enzyme in fatty acid $\beta$-oxidation, was unchanged, as were phosphoenolpyruvate carboxykinase 1 (Pck1; gluconeogenesis) and glucose transporter type 1 and glucose transporter type 4 (Glut1 and Glut4; basal and insulin-mediated glucose transport, respectively).

\section{Effects of OLZ on mitochondrial respiration}

The mRNA expression data suggest that OLZ administration favors glycolysis (Figure 3.4). Previous studies with isolated brain mitochondria have

suggested that OLZ partially inhibits mitochondrial respiration (e.g. $\left.{ }^{92}\right)$. The effect of OLZ exposure on the balance between glycolysis and mitochondrial respiration was therefore determined in vitro in HepG2 cells by Seahorse in both 
glucose- and galactose-containing media (see Experimental Procedures in Chapter II and diagram in Figure 3.5). Under these conditions, proton production rate (PPR) is used as an index of glycolysis, and the oxygen consumption rate (OCR) is used as an index of mitochondrial function (Figure. 3.7). As expected, inhibition of mitochondrial respiration with oligomycin, which forced the cells to rely on anaerobic glycolysis, decreased the rate of OCR, while simultaneously increasing the rate of PPR, thereby greatly increasing the PPR:OCR ratio. Uncoupling the mitochondria with FCCP increased the OCR while still maintaining an elevated PPR, causing the PPR:OCR ratio to decrease relative to oligomycin, but still remain elevated relative to basal (Figure 3.7, panels A, B, C, D, E, and F). In glucose-containing media, OLZ had no apparent effect on OCR or PPR at any concentration. When the Warburg/Crabtree effect was overcome by incubating HepG2 cells in galactose-containing media, ${ }^{88}$ the ratio of PPR:OCR was dramatically decreased, as the cells were forced to rely on oxidative phosphorylation. ${ }^{93}$ Under these conditions, OLZ incubation caused a dosedependent increase in PPR, without significantly affecting OCR (panels $G$ and $H)$.

\section{Effects of OLZ on glucose and glycogen expenditure}

OLZ increased basal (unfasted) plasma glucose, as indicated by plasma taken at sacrifice (Table 3.1). Previous studies have indicated that OLZ administration may cause insulin resistance and/or glucose intolerance. ${ }^{94}$ OLZ also antagonizes serotonin, which is a positive mediator of glycogen synthesis. ${ }^{95}$ To determine if OLZ administration under the current conditions affected glucose 
tolerance, fasted mice were subjected to the OGTT at 25 days of vehicle or drug administration (see Experimental Procedures in Chapter II and Figure 3.7, panel A in this chapter). OLZ exposure significantly increased plasma glucose concentrations 15 minutes after bolus gavage (panel A), but values were similar to controls at all subsequent time points. As mentioned above, the expression of a key inhibitor of glycogen storage (Gsk3b) was significantly increased in livers of OLZ-exposed mice (see Figure 3.4). The effect of OLZ administration on hepatic glycogen storage was therefore assessed by Periodic acid-Schiff reagent (PAS) staining. OLZ administration decreased the amount of glycogen stored in unfasted liver by 2-fold compared to controls (Figure 3.7, panel B, representative photomicrographs, and panel C, quantitative image analysis).

\section{OLZ effects on the hepatic metabolome}

Examining metabolites on an individual basis is time-consuming, and the smaller amounts of data produced may unnecessarily narrow the scope of a study. Metabolomic analysis was therefore used to simultaneously characterize several effects of OLZ on the liver (Figure 3.8). Metabolites that varied significantly between the OLZ group and the control group were then analyzed with pathway analysis software, which predicted and mapped the pathways that may be relevant to OLZ treatment (Figure 3.9). Among other findings, levels of amino acids were significantly affected by OLZ: L-glutamine, a putative mediator of insulin secretion ${ }^{96}$ and mTOR activation, ${ }^{97}$ was increased in animals administered OLZ. Network analysis further predicted the involvement of amino acid transporters SIc7a5 (LAT1) and SIc38a2 (SNAT2), which were recently 
shown to function as regulators of mTOR signaling, ${ }^{97-99}$ and EAAT3, which conversely is regulated by mTOR. ${ }^{100}$

\section{OLZ mediates signaling through mTOR and related pathways}

OLZ administration has been shown to increase peripheral levels of glutamate ${ }^{101,102}$ which can increase the flux of branched chain amino acids (BCAAs) and/or branched chain $\alpha$-ketoacids in tissue. ${ }^{103,104}$ BCAAs, ${ }^{105}$ their $\alpha-$ ketoacid analogs, ${ }^{106}$ and glutamine ${ }^{107}$ have all been identified as potential activators of mammalian target of rapamycin (mTOR) and/or mTOR-dependent signaling cascades. Atypical antipsychotics have also been shown to increase glutamine in the brains of postmortem schizophrenia patients. ${ }^{108}$ Given the key role of mTOR in bioenergetic regulation ${ }^{109}$ and the effects of OLZ on glutamine, (Figure 3.8), mTOR and related proteins were examined by Western blot (Figure 3.10). OLZ administration increased mTOR activation, as indicated by a significant increase in phosphorylation at Ser2448. ${ }^{110}$ OLZ administration also increased phosphorylation of p70S6K at Thr389 and 4EBP1 at Thr37/46, indicating mTORC1 activation, ${ }^{111}$ as well as Akt phosphorylation at Ser473, indicating mTORC2 activation. ${ }^{112}$ Strangely, OLZ treatment concomitantly increased AMPK activation (Figure 3.10), as indicated by an increase in phosphorylation at Thr172. ${ }^{113}$

\section{Discussion}

The serotonin-dopamine antagonist OLZ is a valuable addition to the psychiatric pharmacopeia, but it also presents new challenges for doctors and patients. ${ }^{114}$ Despite the lack of dyskinetic side effects that result from use of 
older antipsychotics, OLZ causes a number of adverse effects that restrict its use, such as obesity and elevated cholesterol. In order for patients to benefit from OLZ without suffering additional metabolic complications, preventive agents and/or treatments must be aimed at the root causes of dysmetabolism during OLZ treatment. Thus far the origins of OLZ dysmetabolism have been elusive, as even the mechanisms that mediate OLZ's desirable therapeutic effects are poorly understood.

The findings from this study - namely, that OLZ activates both AMPK and mTOR, and that this activation may result from altered amino acid transport (see diagram, Figure 3.11) - are therefore important steps toward improving the safety and tolerability of antipsychotic treatment. An earlier intervention proposed for the metabolic side effects of OLZ was metformin, a biguanide drug often prescribed for type II diabetes. Clinical trials of metformin as an adjunct to OLZ therapy have shown disappointingly little improvement in metabolic symptoms, however. ${ }^{115}$ This lack of effect may be due to the fact that AMPK activation is a major mechanism of action for metformin, and AMPK is already activated by OLZ administration.

Targeting mTOR may instead work better than targeting AMPK. It has been suggested that "normalizing" mTOR overactivation could be beneficial for managing obesity and diabetes. ${ }^{116}$ While OLZ-mediated dysmetabolism differs slightly from most cases of metabolic syndrome - a point which is clearly illustrated by the differential activation of AMPK - the effects on mTOR appear similar in both situations. Unlike metformin, then, an mTOR-inhibiting drug such 
as tacrolimus, given at a sub-immunosuppressive dose, may be applicable in both primary and OLZ-mediated metabolic dysfunction.

Future studies will be needed to confirm the therapeutic significance of mTOR suppression as an adjunct to antipsychotic therapy. In certain experimental models, mTOR inhibitors like rapamycin have exacerbated glucose intolerance ${ }^{117}$ and dyslipidemia; ${ }^{118}$ in other studies, mTOR inhibitors have improved metabolic homeostasis. ${ }^{119-121}$ Appropriate dosing appears crucial to the ultimate outcome of treatment with mTOR inhibitors. ${ }^{122}$ It should also be determined what effects, if any, mTOR inhibitors have on mental functioning and overall OLZ efficacy. The glutamatergic model of mental illness proposes that antipsychotic disorders are disorders of excitatory amino acid metabolism. ${ }^{123}$ Targeting mTOR, then, would be arguably "downstream" of OLZ's psychiatric effects. 
Table 3.1: Plasma parameters after 4 weeks of OLZ treatment

\begin{tabular}{llll}
\hline Glucose & $(\mathrm{mg} / \mathrm{dL})$ & Control & OLZ \\
\hline TG & $(\mathrm{mg} / \mathrm{dL})$ & $42 \pm 2$ & $329 \pm 13^{*}$ \\
Cholesterol & $(\mathrm{mg} / \mathrm{dL})$ & $50 \pm 6$ & $80 \pm 7^{*}$ \\
HDL & $(\mathrm{mg} / \mathrm{dL})$ & $34 \pm 4$ & $62 \pm 3$ \\
LDL & $(\mathrm{mg} / \mathrm{dL})$ & $8 \pm 2$ & $42 \pm 4$ \\
VLDL & $(\mathrm{mg} / \mathrm{dL})$ & $9 \pm 1$ & $4 \pm 2$ \\
ALT & $(\mathrm{U} / \mathrm{L})$ & $33 \pm 9$ & $16 \pm 1^{*}$ \\
AST & $(\mathrm{U} / \mathrm{L})$ & $56 \pm 2$ & $76 \pm 9^{*}$
\end{tabular}

Animals and treatments are described in Experimental Procedures. Data are means \pm S.E.M. $(n=4-8)$ and are reported as indicated in the individual rows. ${ }^{*}, P<.05$ compared to no OLZ treatment. 


\title{
Figure 3.1: Experimental timeline
}

\author{
Mice were switched to purified TD.08485 diet one week prior to OLZ \\ administration ( $8 \mathrm{mg} / \mathrm{kg} / \mathrm{d}$, s.c.). Oral glucose tolerance testing (OGTT) was \\ performed after 25 days of OLZ administration. After four weeks of continuous \\ OLZ, mice were sacrificed as described in Chapter II.
}




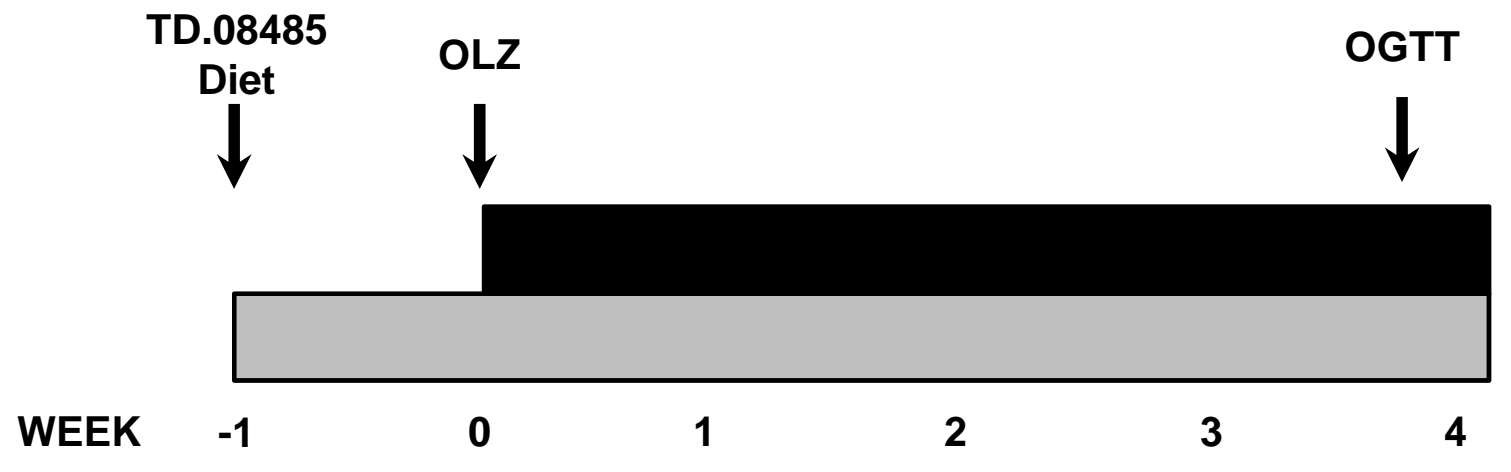




\section{Figure 3.2: Effect of OLZ on body weight gain and adiposity}

Mice were treated with OLZ for four weeks as described in Chapter II. Average weekly food consumption and weight gain are shown (panel A). Body fat as a percentage of total body weight was measured by x-ray absorptiometry and gonadal fat pads were removed and weighed at sacrifice (panel B). Data are means \pm S.E.M. $(n=4-8) .{ }^{*}, P<.05$ compared to no OLZ treatment. 
A.

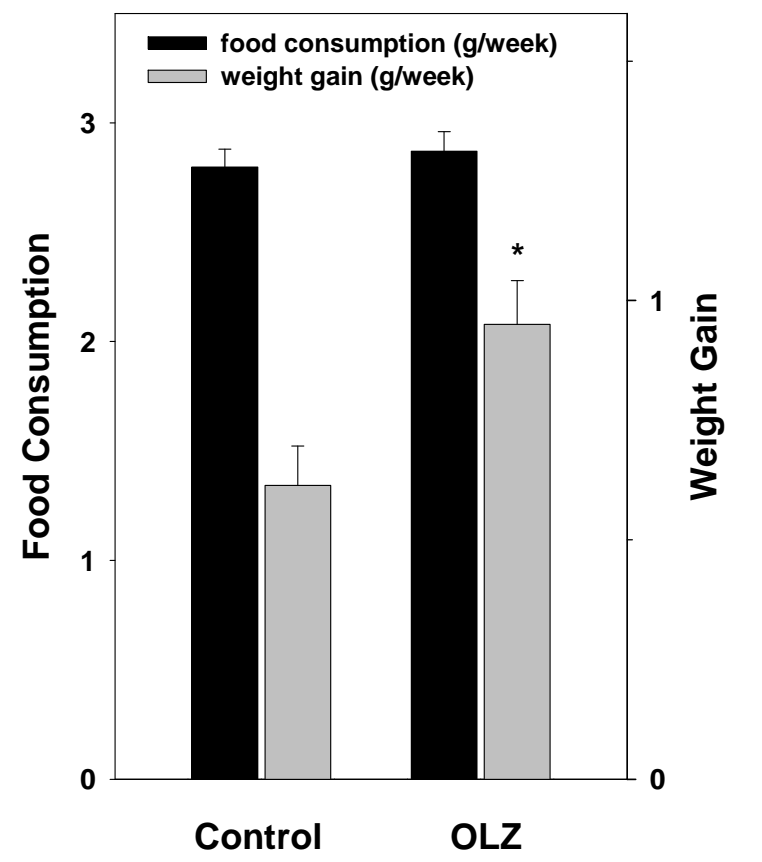

B.

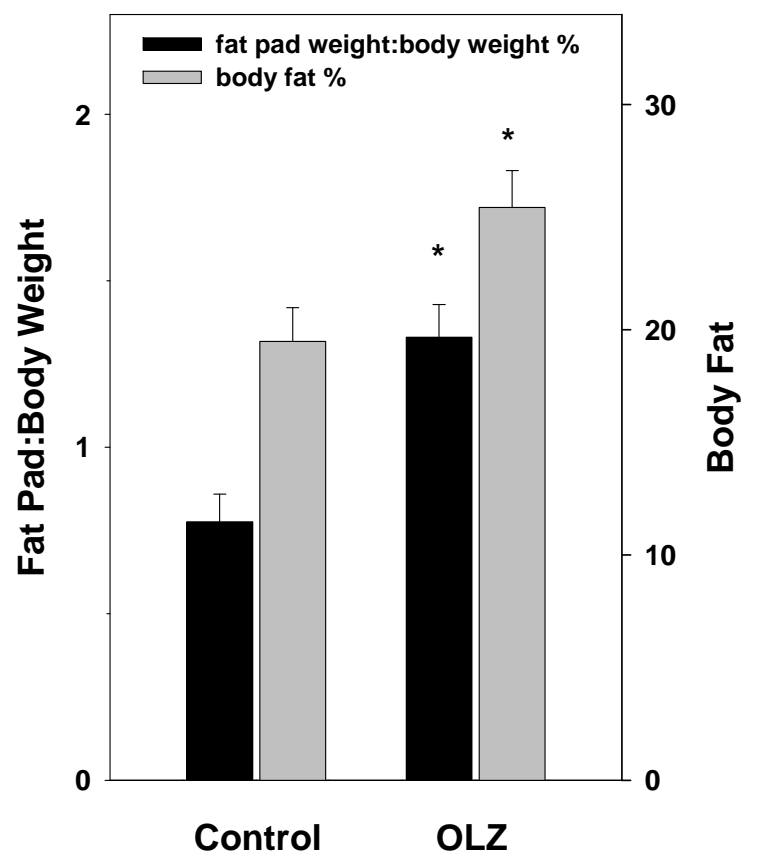




\section{Figure 3.3: OLZ promotes hepatic lipid accumulation}

General histology is represented by hematoxylin and eosin staining (panel A, top). Neutral lipid accumulation is shown with Oil Red O staining (panel A, bottom). Hepatic triglyceride (TG) and non-esterified fatty acid (NEFA) contents (panel B) were determined after chloroform:methanol extraction as described in Experimental Procedures. Liver weight measurements taken at sacrifice are expressed as a percentage of total body weight, also measured at sacrifice (panel C). Data are means \pm S.E.M.(n=4-8). ${ }^{*}, P<.05$ compared to no OLZ treatment. 
A

Control
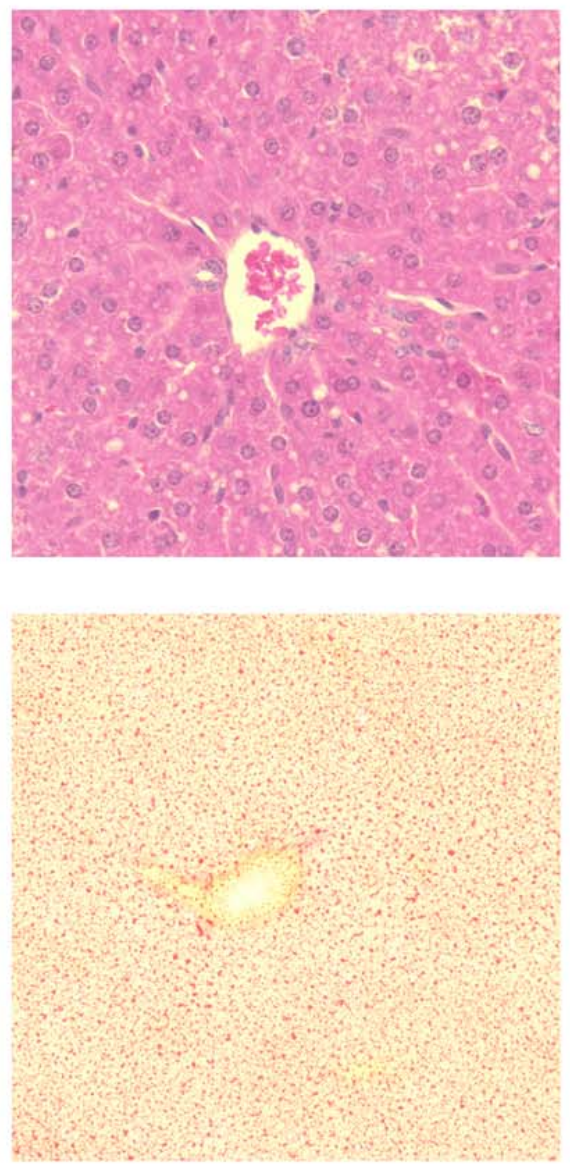

B

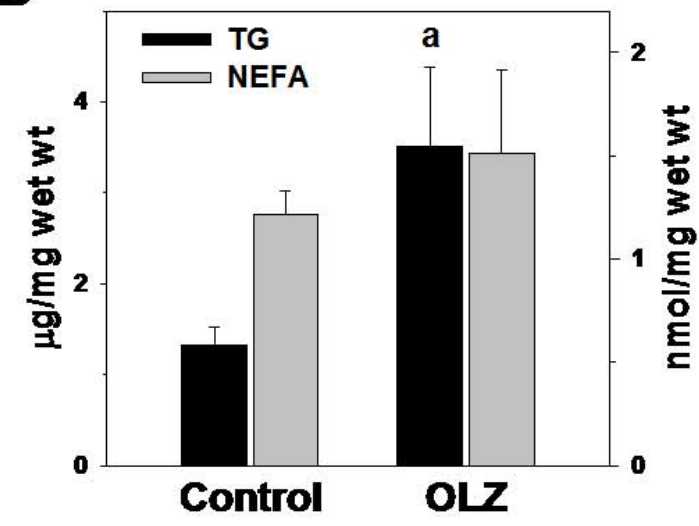

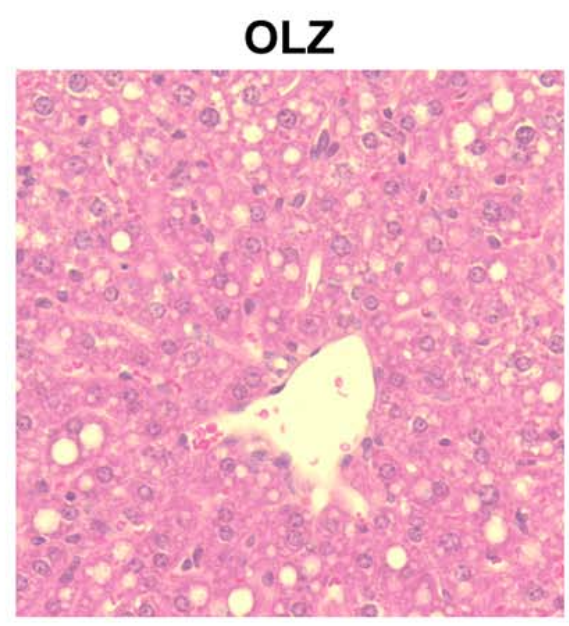

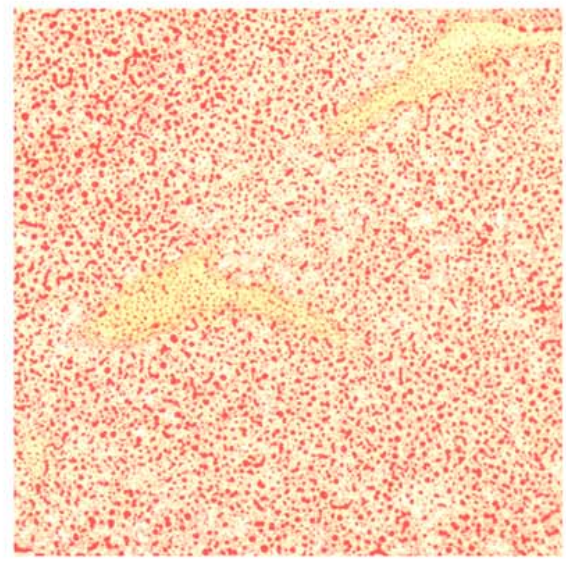

C

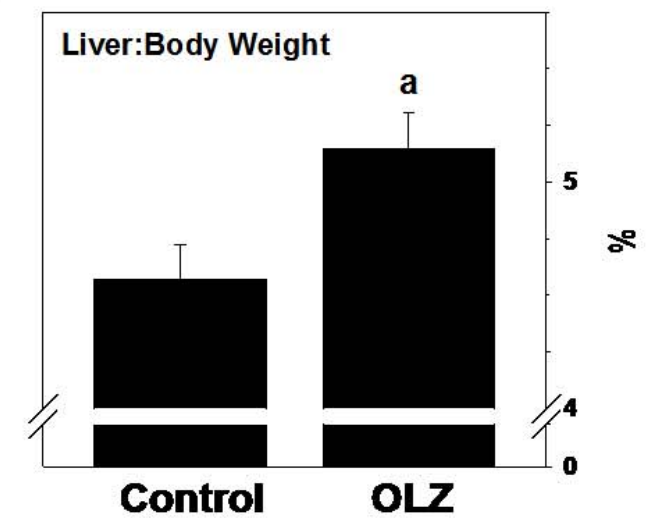


Figure 3.4: Effects of OLZ on hepatic gene expression

Hepatic mRNA expression of genes for lipid metabolism (top) and carbohydrate metabolism (bottom) was determined by qRT-PCR after four weeks of continuous OLZ treatment, as described in Experimental Procedures. Data are means \pm S.E.M. $(\mathrm{n}=4-8) . \quad{ }^{*}, P<.05$ compared to no OLZ treatment. 


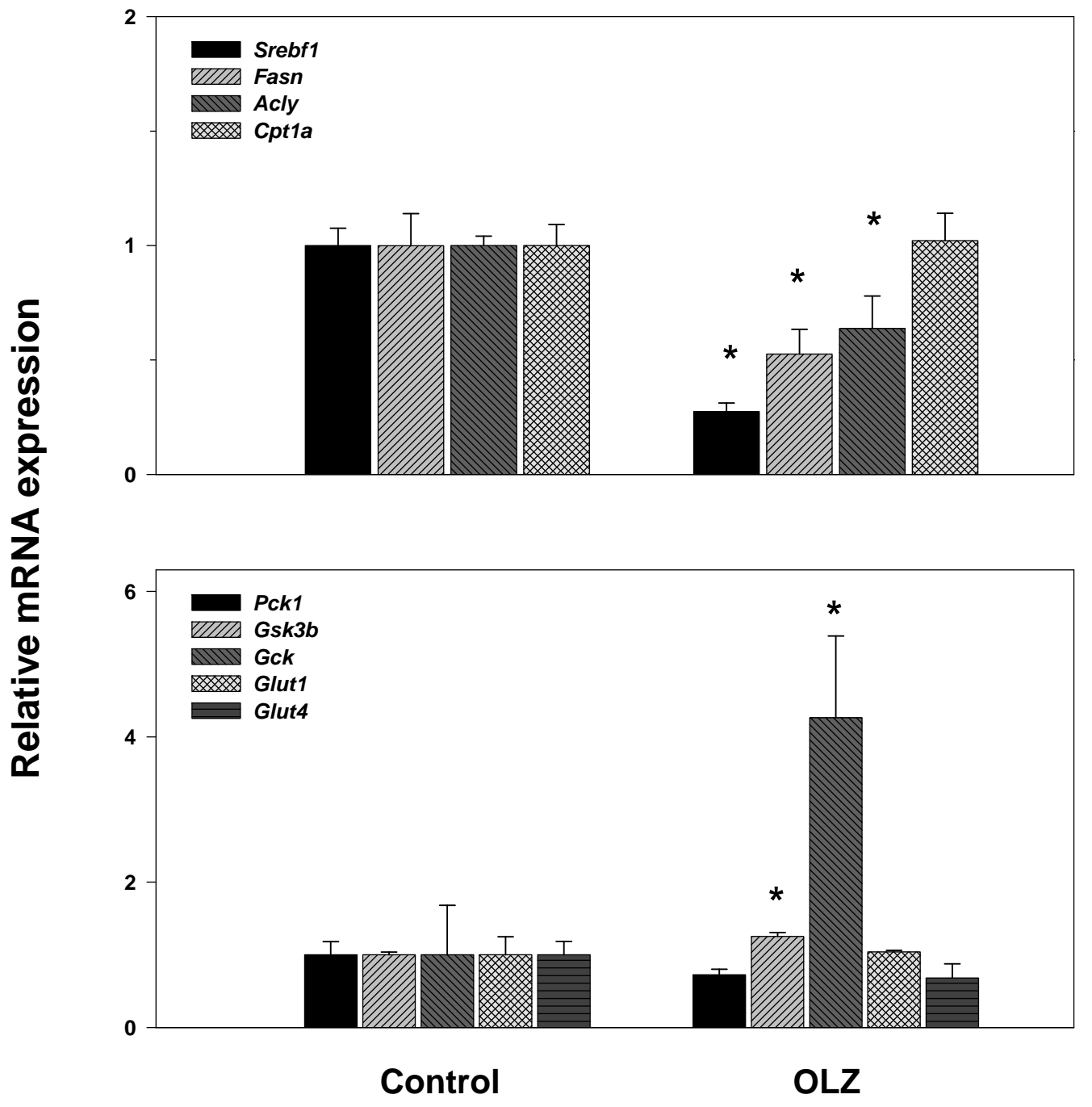


Figure 3.5: Glucose vs. galactose media for immortalized cells

\author{
Panel A: In primary cells ("normal" cells), energy requirements are mostly \\ met by producing ATP via oxidative phosphorylation (OXPHOS), with glycolysis \\ making a small contribution to overall energy status. Immortalized cells, in \\ contrast, primarily generate ATP through glycolysis. Panel B: Because \\ metabolism of galactose produces no net ATP, immortalized cells must rely on \\ OXPHOS to fulfill ATP needs.
}


A.
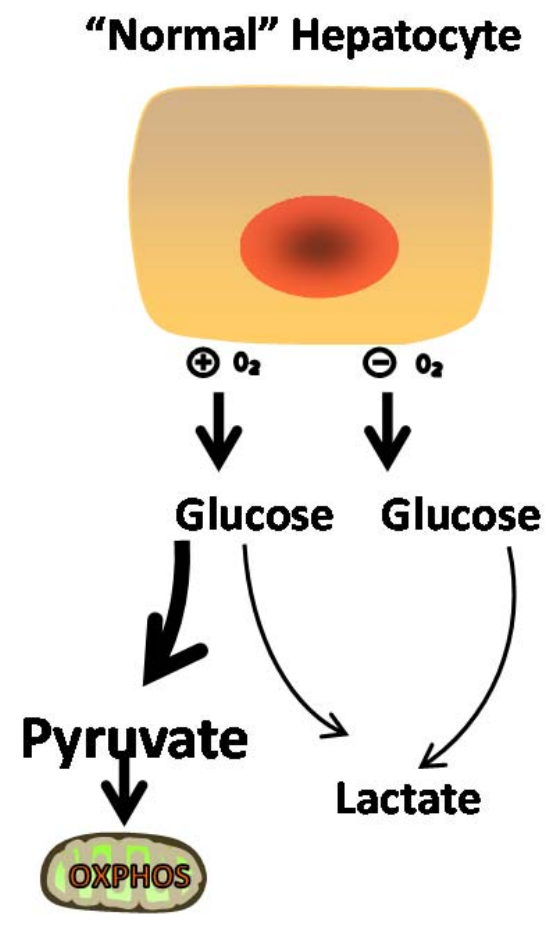

B. "Normal" Hepatocyte
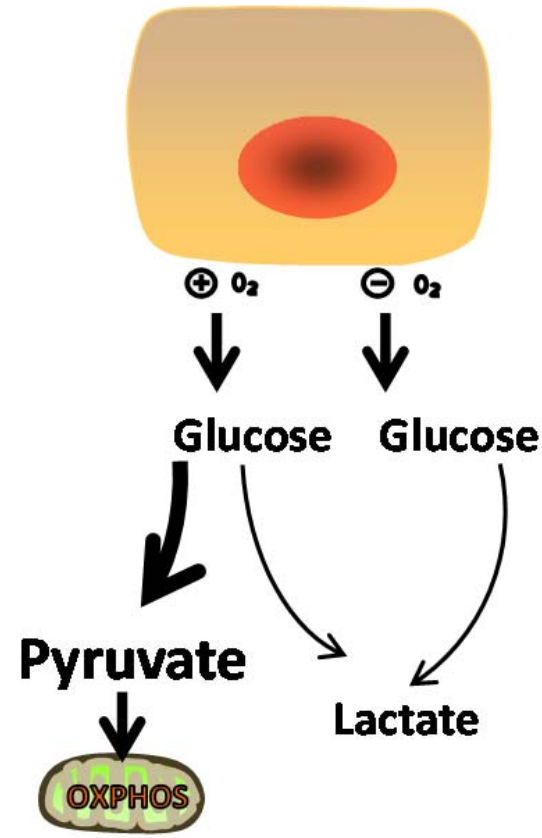

HepG2 Cell (Immortalized)

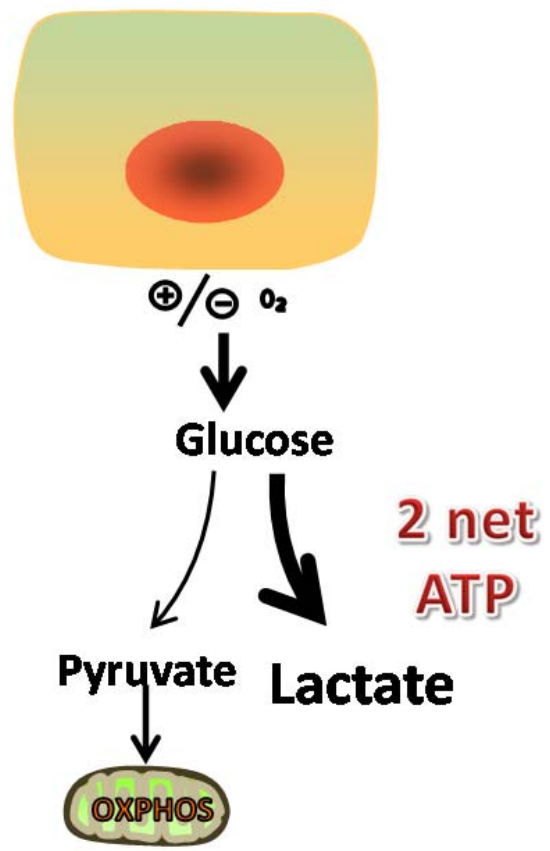

HepG2 Cell (Immortalized)

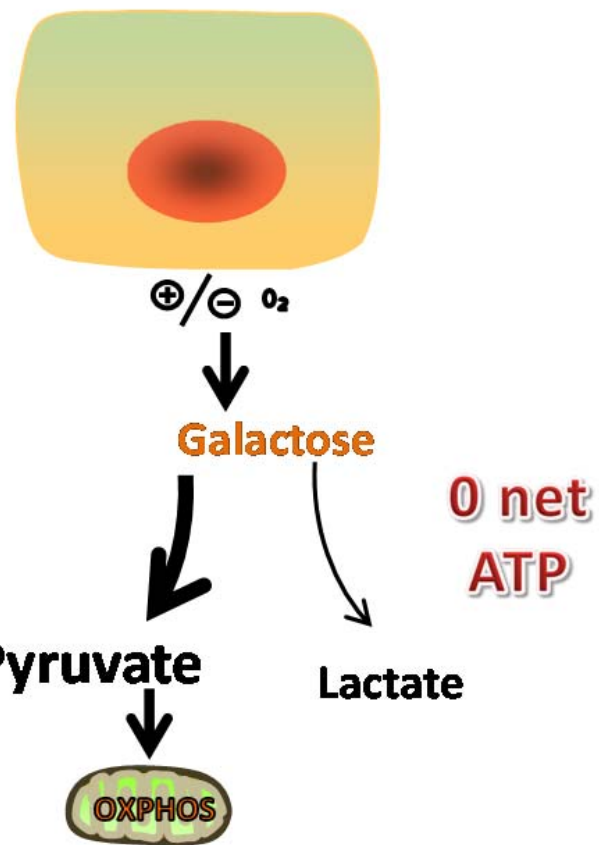


Figure 3.6: In vitro metabolism of OLZ-treated HepG2 cells

Respiration was measured in HepG2 cells grown in either glucose or galactose and graded concentrations of OLZ $(0-25 \mu \mathrm{M})$. PPR (panels A and B), OCR (panels C and D), and PPR:OCR (panels E and F) are shown as a function of time for 0 and $25 \mu \mathrm{M} \mathrm{OLZ}$, with both glucose and galactose media. Measurements of PPR and OCR are also shown for galactose-grown cells in panels $\mathrm{G}$ and $\mathrm{H}$ Basal $=10$ minutes before the addition of oligomycin; Oligo $=10$ minutes after the addition of oligomycin. ${ }^{*}, P<.05$ compared to no OLZ treatment. 
A.

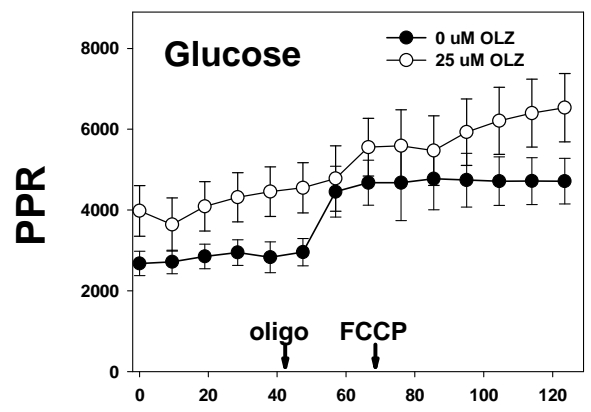

c.

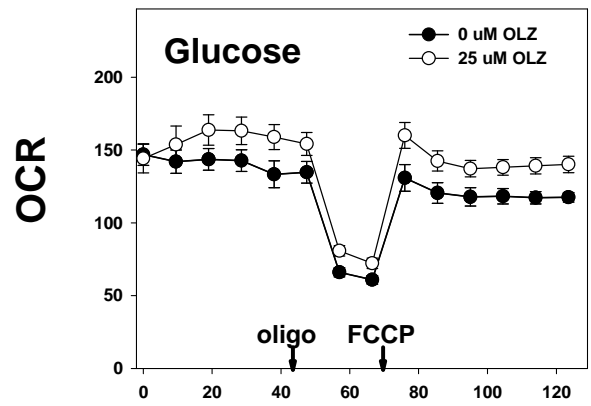

E.

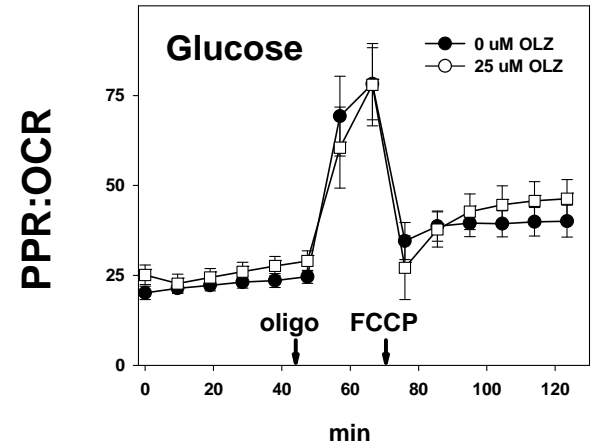

G.

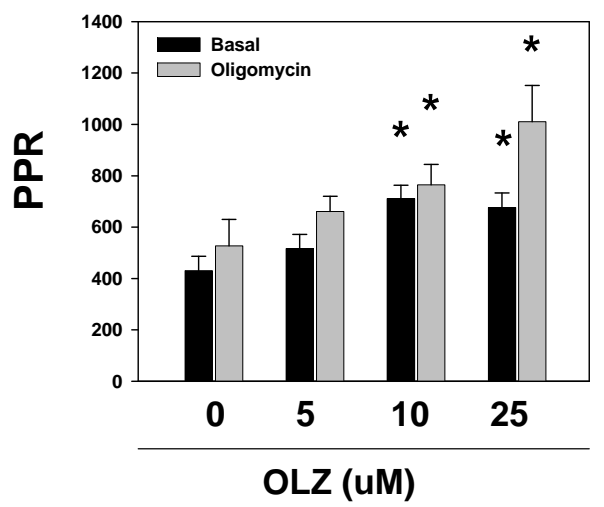

B.

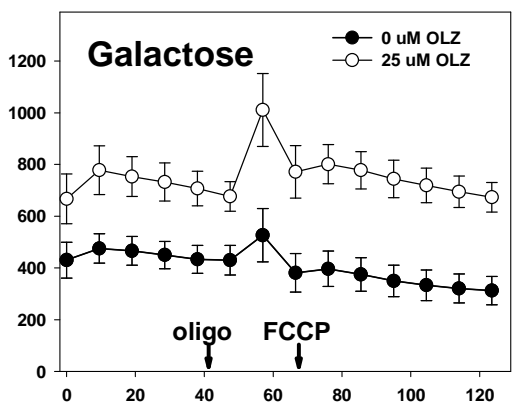

D.

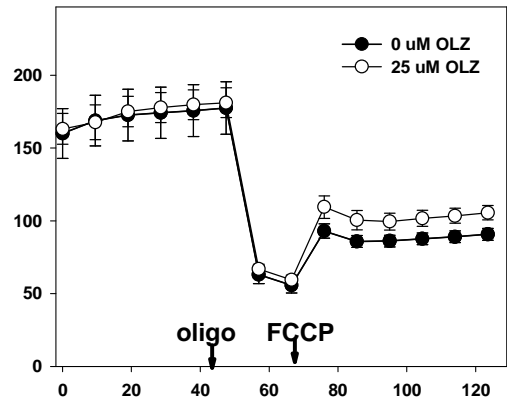

F.
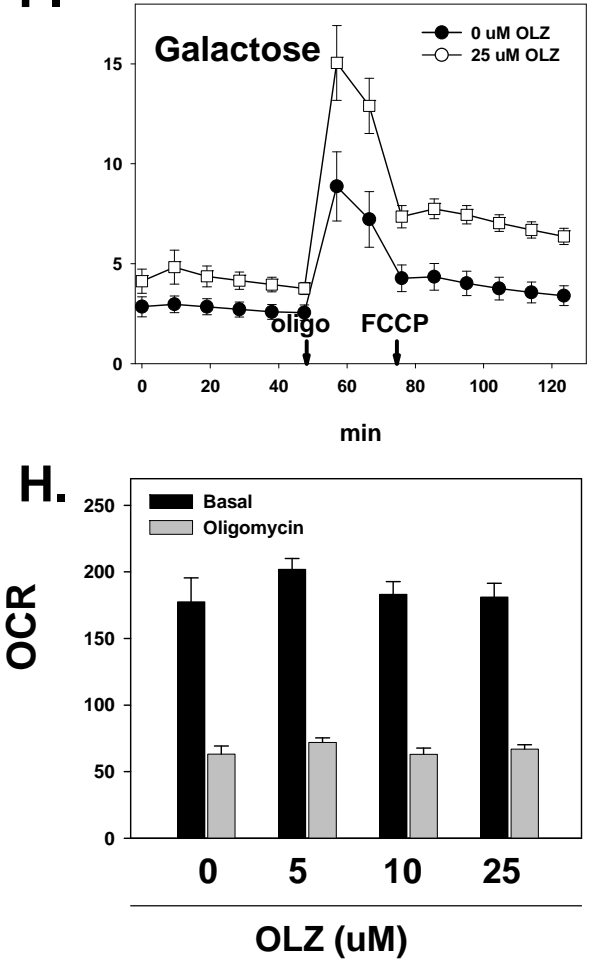
Figure 3.7: Effects of OLZ on glucose and glycogen expenditure

\begin{abstract}
Glucose tolerance (panel A) was assessed as described in Experimental Procedures. Liver sections were stained with Periodic acid-Schiff reagent and representative photomicrographs are depicted (panel B). PAS staining was quantitated (panel C) as described in Chapter II. Data are means \pm S.E.M. $(n=4-$ 8). ${ }^{*}, P<.05$ compared to no OLZ treatment.
\end{abstract}



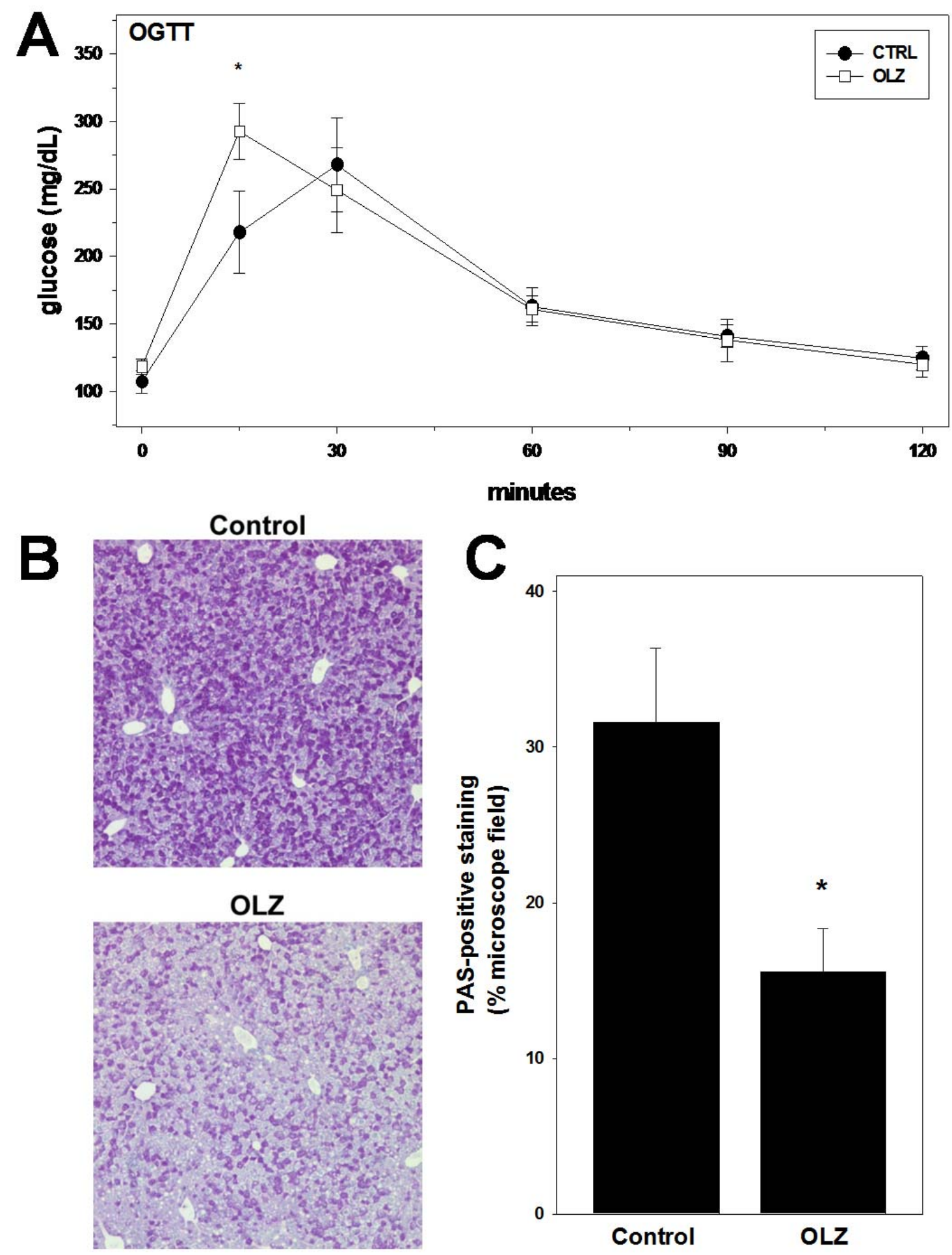
Figure 3.8: OLZ effects on the hepatic metabolome

Liver samples were derivatized as described in Chapter II and metabolites determined by GC×GC-TOF MS. Relative abundance data is shown for selected small molecule metabolites. Data are means \pm S.E.M. $(\mathrm{n}=4-8) .{ }^{*}, P<.05$ compared to no OLZ treatment. 
OLZ compared to CONTROL

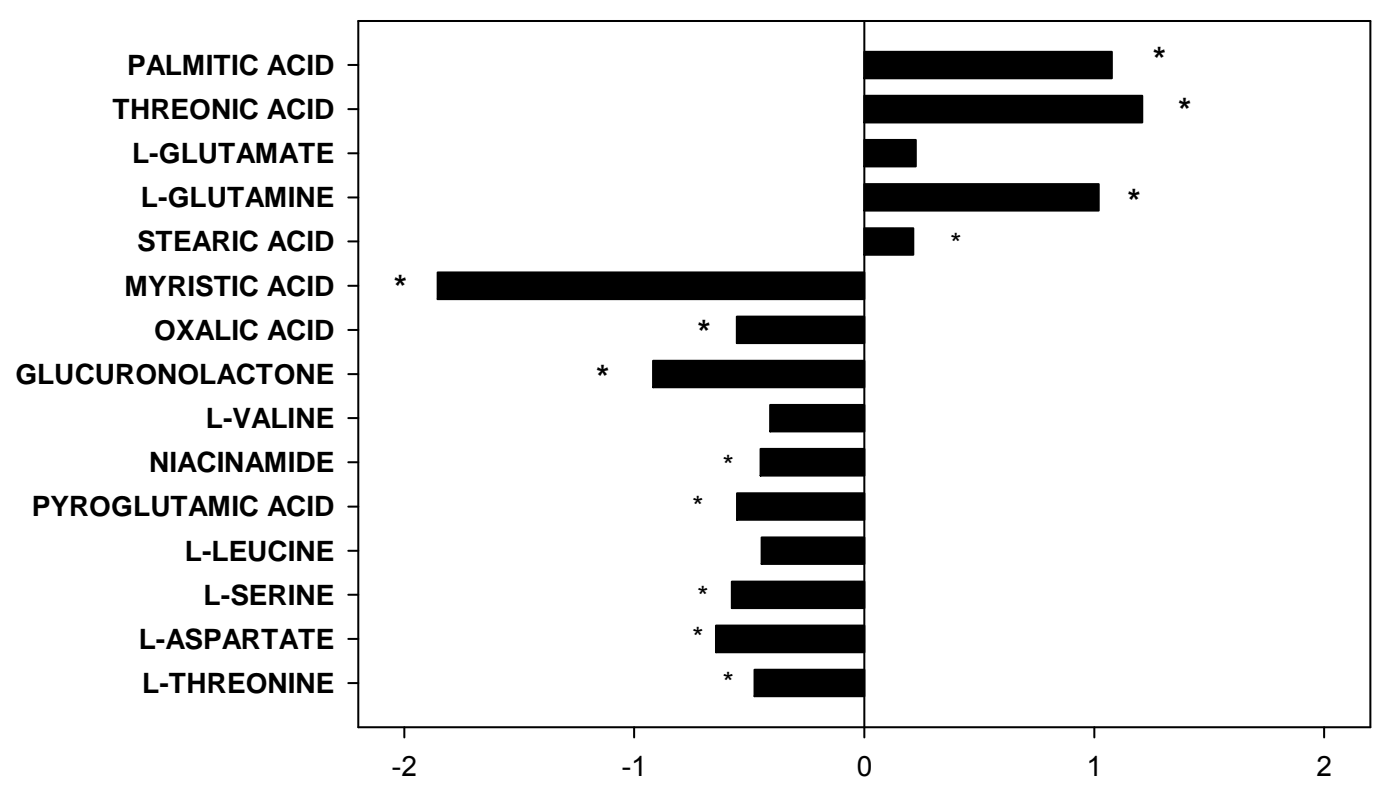

Fold change vs control (Log2) 


\section{Figure 3.9: Metabolic pathways}

Metabolites identified by GC×GC-TOF MS were evaluated with

MetaCore ${ }^{\mathrm{TM}}$ software and used to construct a pathway map. Targets related to mTOR and amino acid metabolism are enlarged and shown beneath the map. 


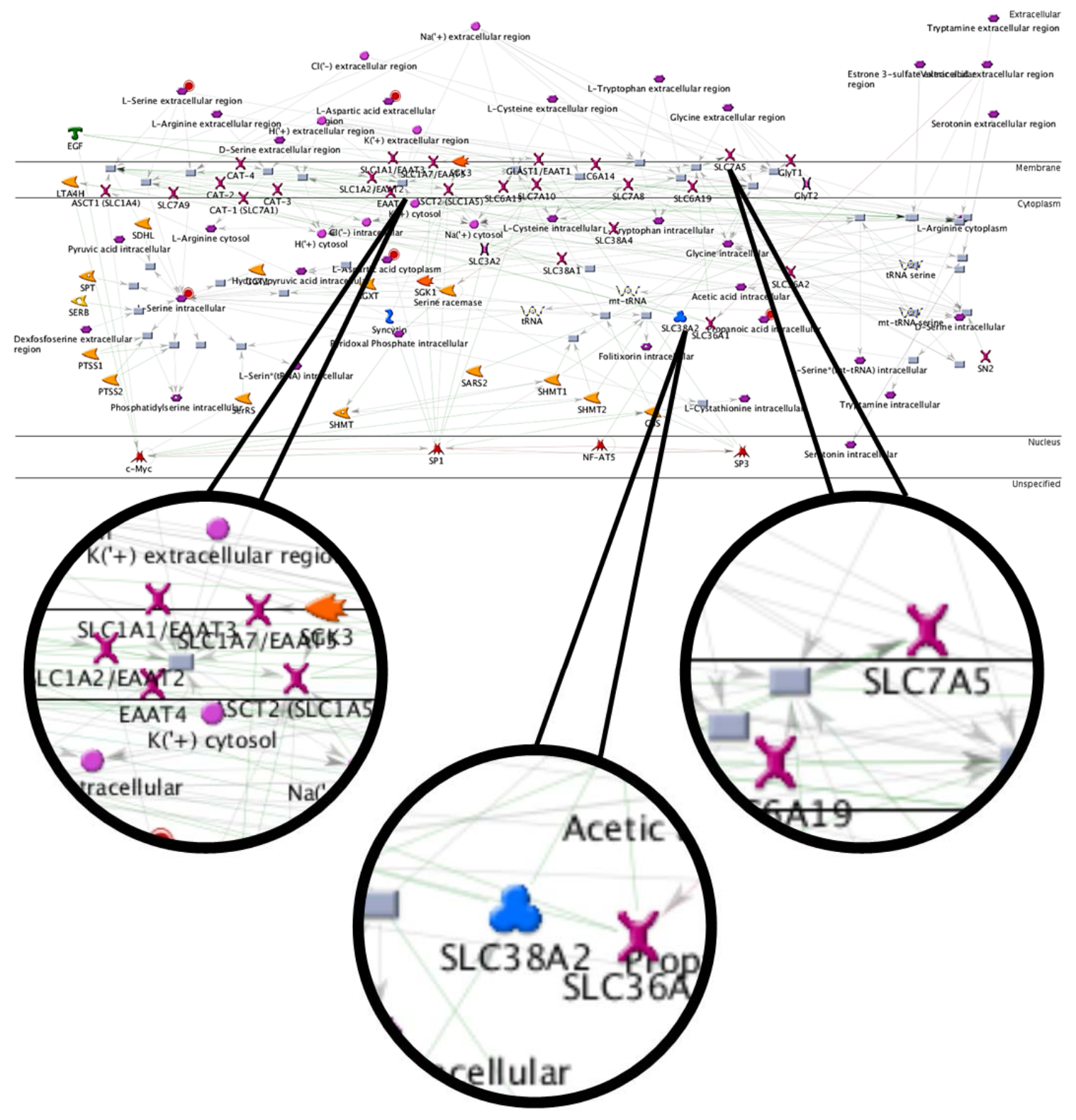

LEGEND

Protein


Figure 3.10: OLZ mediates signaling through mTOR and related pathways

\author{
Liver homogenates were prepared as described in Experimental \\ Procedures, Chapter II. Representative immunoblots are shown (panel A). \\ Quantitative analysis was performed and the ratio of phosphorylated to total \\ protein is shown in (panel B). ${ }^{*}, P<.05$ compared to no OLZ treatment.
}


A

phospho

total

mTOR

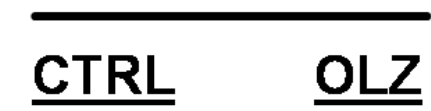

CTRL

$\underline{\mathrm{OLZ}}$

p70s6k
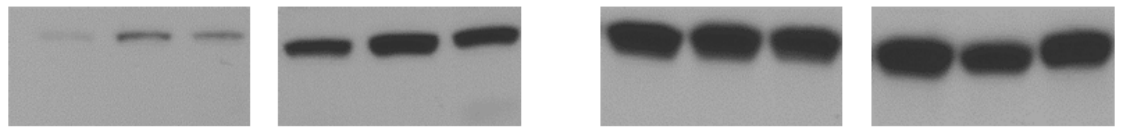

4EBP1
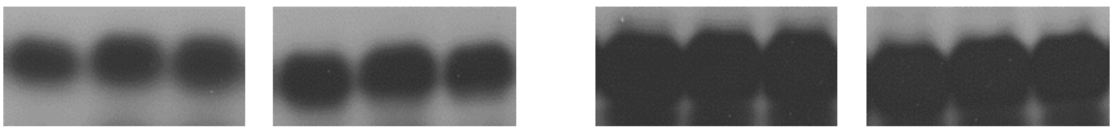

Akt
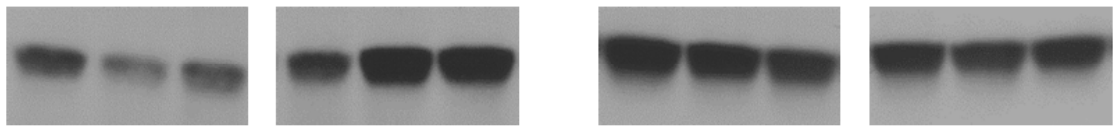

\section{AMPK}
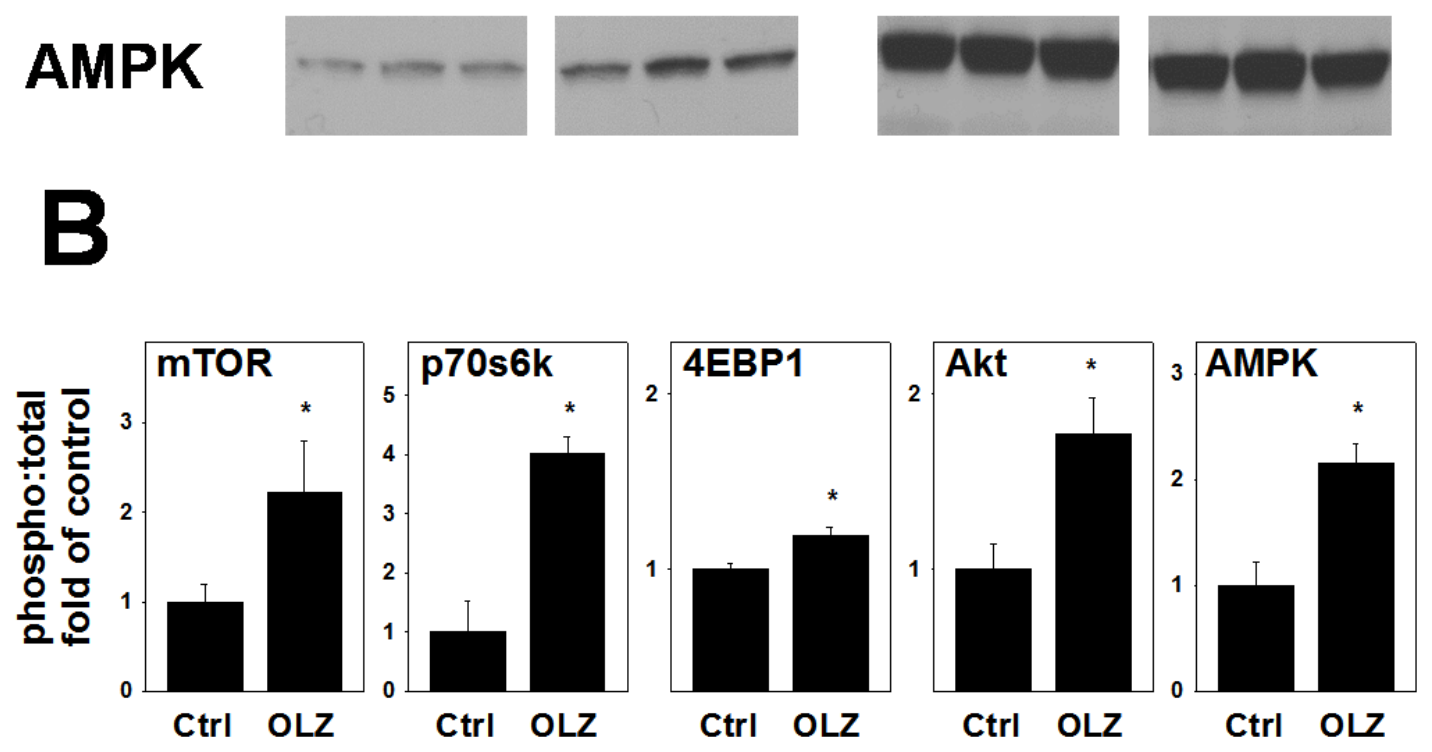
Figure 3.11: Hypothesized mechanism of OLZ-induced metabolic dysregulation in liver

OLZ promotes flux of glutamine (GIn) and leucine (Leu) through solute carrier 38a2 (SLC38A2 or SNAT2) and solute carrier 7a5 (SLC7A5 or LAT1). Increased concentrations of Gln and Leu induce mTOR signaling. Excess intracellular Leu acts as a nitrogen donor in the branched chain aminotransferase (BCAT) reaction that forms glutamate (Glu). Leu and Glu also contribute metabolic intermediates (e.g., acetyl CoA) to the Krebs cycle. Interestingly, AMPK was simultaneously activated under these conditions (see discussion in Chapter VI for additional information). 


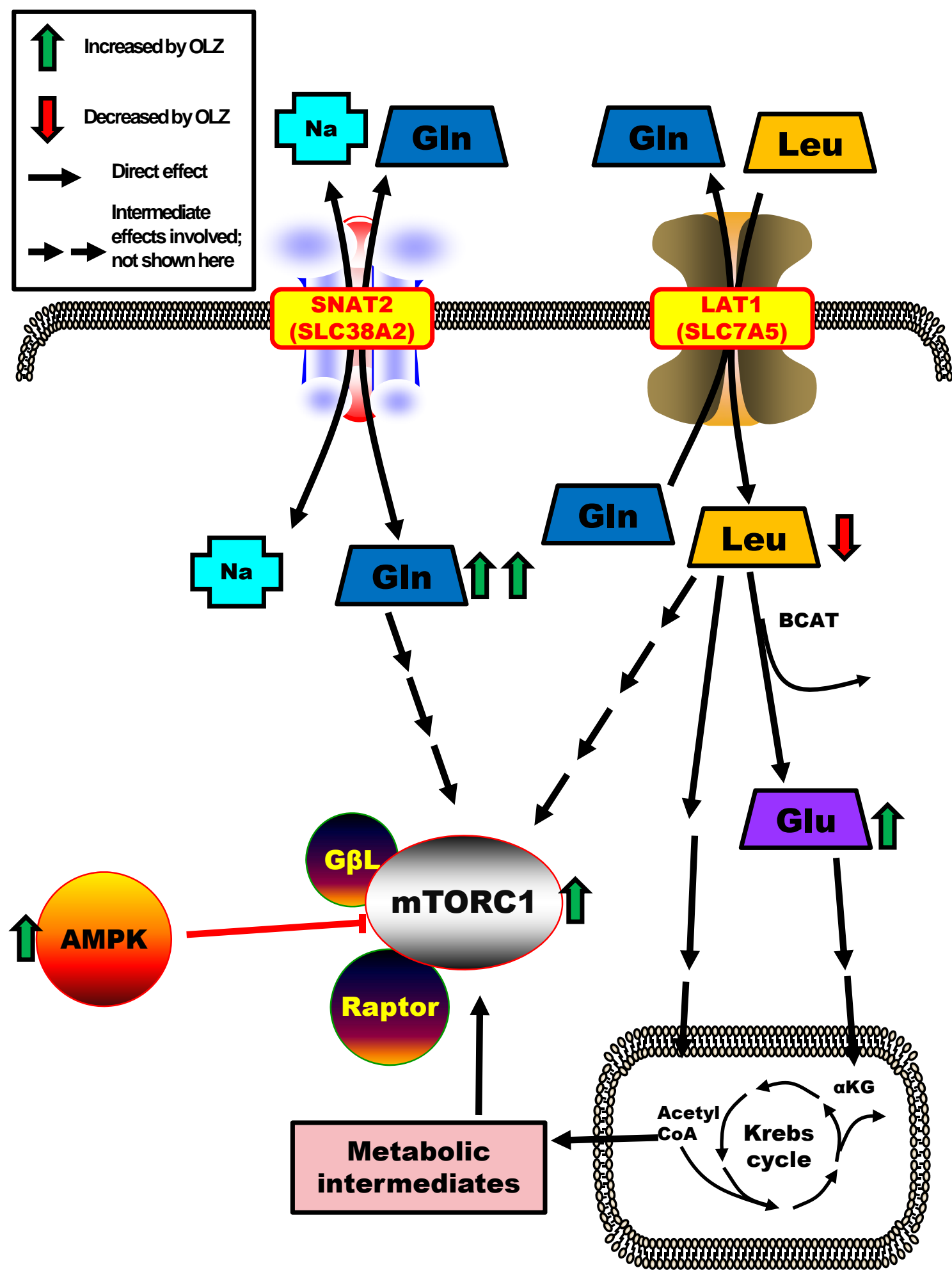




\section{CHAPTER IV}

\section{HIGH-FAT DIET EXACERBATES OLANZAPINE-INDUCED LIVER INJURY}

\section{A. Introduction}

In the preceding sections of this dissertation it was noted that weight gain and obesity caused by OLZ are serious health risks as well as barriers to successful treatment of mental illness. OLZ administration causes weight gain in normal-weight individuals; OLZ use may then be especially detrimental for overweight patients or patients who consume high-fat, high-calorie diets. Indeed, OLZ has been shown to "unmask" metabolic syndrome and type II diabetes in previously asymptomatic individuals. ${ }^{32,124,125}$ The purpose of these experiments, therefore, was to determine the interactions that result from concurrent OLZ administration and HFD feeding, and to isolate the unique pathological features of these interactions.

\section{B. Experimental Procedures}

\section{Animals and treatments}

Mice were separated into four groups ( $2 \times 2$ factorial design), receiving either TD.08485 low-fat or TD.88137 high-fat diet and either OLZ or vehicle control. Diets were started one week before the four week drug administration 
period. Drug administration and sacrifice procedure are described in greater detail in the Experimental Procedures chapter (Chapter II).

\section{Oral glucose tolerance test (OGTT)}

Glucose tolerance testing was performed as described in Chapter II.

\section{Glycogen determinations}

Glycogen was measured in liver tissue aliquots as described in Chapter II.

\section{RNA isolation and real-time RT-PCR}

Details for RNA isolation and PCR procedure are found in Chapter II.

\section{Biochemical analyses and histology}

Liver sections were stained with H\&E, Oil Red O, CAE, PAS, TUNEL and 4- HNE as described in Chapter II.

\section{Lipid determinations}

Hepatic lipids were measured as previously discussed in Chapter II.

\section{Results}

1. High-fat diet (HFD) increases food consumption and exacerbates weight gain caused by OLZ

All animals gained weight over the course of the study and no mortality was observed in any group. Mice fed HFD consumed more food and gained more weight on average than mice fed LFD (Figure 4.2, panels A and B). OLZ did not have any effect on food consumption, but added to body weight gain in both diet groups. OLZ also increased the ratio of gonadal fat mass to total body 
mass, which was further increased by concurrent HFD feeding (panel C). OLZ and HFD promoted overall body fat accumulation as shown by DEXA scan (representative images, panel D).

\section{HFD aggravates OLZ-induced liver injury}

Previous studies have shown that feeding mice a diet enriched in triglycerides and cholesterol (i.e., the "Western diet") damages the liver and may contribute to the onset of NAFLD. Studies from this laboratory have also linked OLZ administration to early indices of liver damage. Liver injury was indeed observed in OLZ-treated animals, and this liver injury was aggravated by concurrent HFD feeding. As in previous work, four weeks of OLZ increased fat accumulation across all regions of the liver, as shown by H\&E staining (Figure 4.3, panel A, top row). Co-administration of HFD further increased the fat accumulation resulting from OLZ alone. Neutrophil infiltration in liver was increased by HFD or OLZ alone, and more so by HFD and OLZ together (representative pictures in bottom row of panel A and cell counting, panel D). OLZ administration elevated circulating hepatic transaminases, a common indicator of hepatocyte death (panels B and C). HFD alone had no effect on transaminase levels; however, in conjunction with OLZ, HFD raised ALT and AST to levels greater than OLZ alone. OLZ and HFD separately increased the liver weight to body weight ratio, but the combination of OLZ and HFD produced no additional change (panel E).

\section{OLZ and HFD promote hepatic lipid storage}


Hematoxylin and eosin staining had demonstrated steatosis with OLZ and HFD both alone and in combination. Therefore, frozen liver sections were stained with Oil Red O (Figure 4.4, panel A) and staining intensity (i.e., extent of lipid accumulation) was quantitated (panel B). Minimal staining intensity was seen with LFD feeding, indicating very little hepatic fat accumulation. OLZ administration caused a 1.5-fold increase in micro- and macrovesicular steatosis, as determined by image analysis. HFD by itself caused a 2 -fold increase in steatosis. When OLZ and HFD were administered together, steatosis increased 2.5-fold compared to LFD alone. Hepatic lipid extraction elaborated upon these Oil Red O data: OLZ and HFD separately doubled triglyceride (TG) content in liver, and together increased TG fourfold (panel C). Hepatic free fatty acids (NEFA) were elevated with HFD alone, but remained stable in all other treatment groups.

\section{Effects of OLZ and HFD on glucose metabolism}

In vivo glucose metabolism was measured by oral glucose tolerance test (OGTT) after 25 days of drug administration and LFD or HFD consumption (see Experimental Procedures for additional details). Four weeks of OLZ and/or HFD did not significantly alter plasma glucose concentrations after a 6 hour fast (Figure 4.5, panel A). Following bolus glucose administration, however, plasma glucose was markedly elevated in both HFD- and OLZ-exposed animals. Exposure to OLZ and/or HFD also influenced glucose kinetics. In mice given OLZ and HFD, either separately or in combination, peak plasma glucose was observed at 15 minutes in contrast to 30 minutes for LFD-fed controls. 
Interestingly, at the 30 minute time point, plasma glucose remained elevated in mice given HFD alone, but rapidly declined in mice given HFD and OLZ together.

\section{HFD worsens OLZ-induced glycogen depletion}

A previous study by this group showed that OLZ exposure attenuated glycogen storage in liver. Here, the interaction between OLZ and HFD was investigated. Periodic acid-Schiff (PAS) staining showed that OLZ alone and HFD alone decreased hepatic glycogen reserves (Figure 4.5, panels B and C). Glycogen was additionally depleted by co-administration of OLZ and HFD.

\section{Effects of HFD and OLZ on apoptosis and lipid peroxidation}

To better determine the cause of aggravated liver injury with concurrent HFD and OLZ, liver sections were stained for markers of apoptosis and lipid peroxidation. Though few apoptotic cells were counted in any treatment group, HFD alone doubled the number of TUNEL-positive cells per 1,000 hepatocytes counted (Figure 4.6, panels A and B). No additional change was seen with HFD and OLZ in combination. Lipid peroxidation increased $\sim 6$-fold with OLZ alone, and $\sim 8$-fold with HFD and OLZ in combination (panel C).

\section{Discussion}

According to the "two hit" hypothesis, now widely accepted in the field of hepatology, liver disease development requires two or more compounding factors. The first factor, or "hit," increases sensitivity to injury by later insults. For example, injecting mice with lipopolysaccharide (LPS) provokes an immune response and produces mild liver injury. If the same mice are exposed to alcohol 
shortly afterward, liver injury becomes exponentially worse. ${ }^{126}$ The order of these two hits is probably not as important as the fact they occur in succession. In an experiment by Yang et al. using obese (fa/fa) Zucker rats, LPS functioned as a second hit; the widespread hepatic steatosis already present in this genetic strain is considered to represent the first hit. ${ }^{48}$

In the experiments reported in Chapter IV of this work, a different but complementary two-hit phenomenon was observed. OLZ produced toxic effects with both high- and low-fat diets, but the combined effects of OLZ and HFD noticeably exacerbated toxicity, increasing fat accumulation and inflammatory response, among other observable injuries. Oxidative stress also markedly worsened during HFD + OLZ co-exposure. These results parallel both Yang's work and much subsequent research. The HFD + OLZ model, in demonstrating multiplied injury, maintains rather than innovates an important toxicological concept.

The importance of the HFD + OLZ model instead arises from its implications for two overlapping clinical populations. As mentioned in Chapter I, a majority of Americans today are overweight. Of this overweight population, nearly half are obese $(\mathrm{BMI} \geq 30)$. Rates of overweight and obesity have increased over the last 50 years and show no evidence of decline. While the prevalence of schizophrenia and affective disorders is much smaller $(\sim 1$ $4 \%),{ }^{127,128}$ the large and expanding proportions of the national weight problem increase the likelihood of a hepatotoxic two-hit interaction. 
Taken together, the results from this study illustrate the concept that more serious liver diseases may require multiple factors in their progression. This demonstrates the need for additional monitoring of overweight or obese patients who also take olanzapine. Also, because oxidative stress and inflammation were shown to worsen with HFD and OLZ, potential pharmacological interventions should be aimed at suppressing oxidative injury and/or exaggerated inflammatory response. 


\section{Figure 4.1: Experimental timeline}

Mice were switched to purified low- or high-fat diets (LFD or HFD) one week prior to OLZ administration (8 mg/kg/d, s.c.). Oral glucose tolerance testing (OGTT) was performed after 25 days of OLZ administration. After four weeks of continuous OLZ, mice were sacrificed as described in Chapter II. 


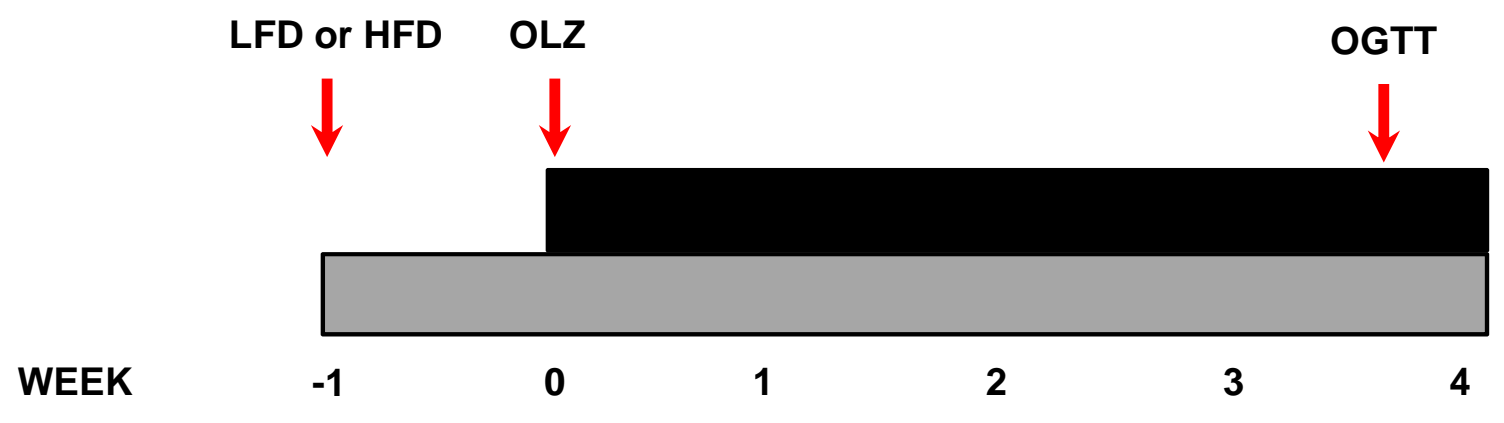




\section{Figure 4.2: High-fat diet (HFD) increases food consumption and exacerbates weight gain caused by OLZ}

Mice were fed HFD or LFD and administered OLZ or vehicle by osmotic minipumps for four weeks. Food consumption and weight gain over the course of the four week study is shown in panels A and B. Gonadal fat pads were removed at sacrifice and percentage of gonadal fat mass to total body mass was determined (panel C). Body composition was measured by dual energy $\mathrm{x}$-ray absorptiometry; representative scans are depicted (panel D). Data are means \pm S.E.M. $(\mathrm{n}=4-8) .{ }^{\mathrm{a}}, P<0.05$ effect of HFD; ${ }^{b}, P<0.05$ effect of $\mathrm{OLZ}$ as determined with two-way ANOVA and Holm-Šidák multiple comparison test. 


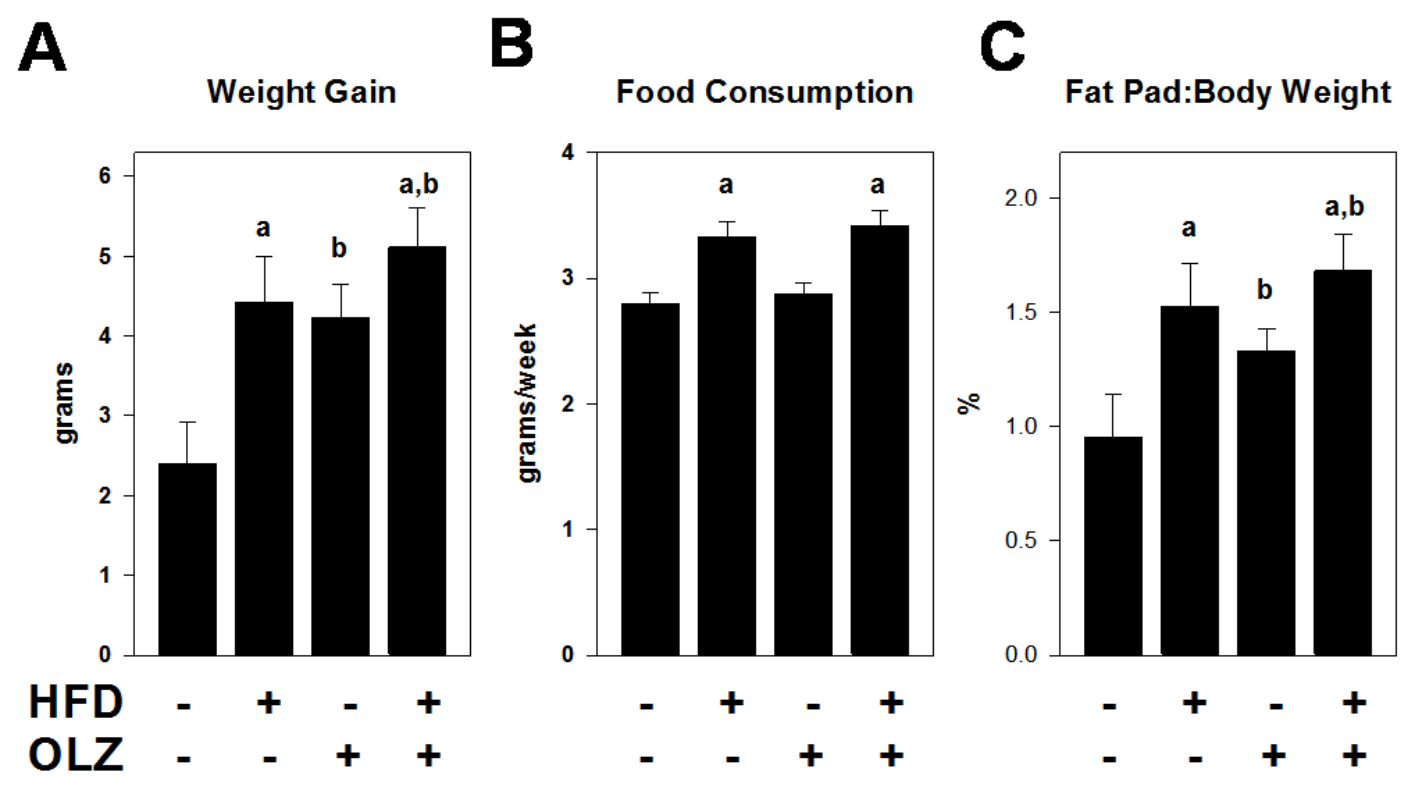

D
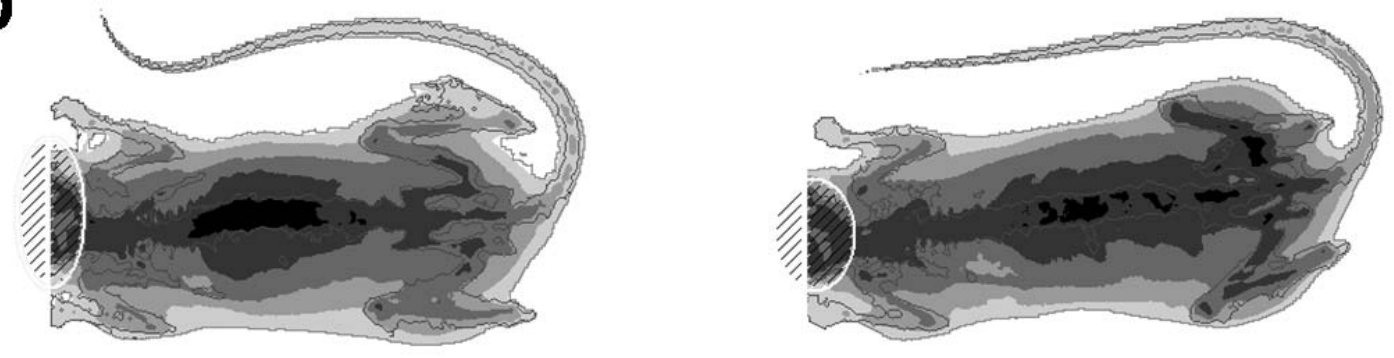

LFD $-16.6 \%$
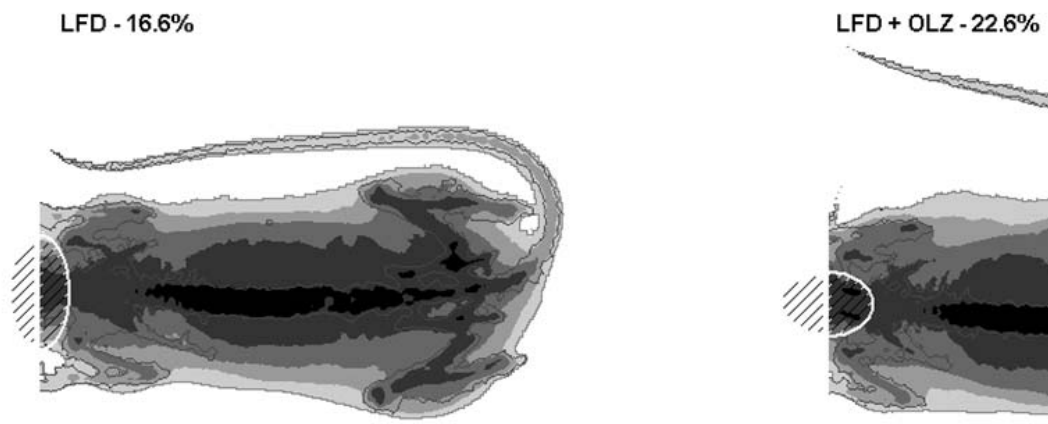

HFD - $24.8 \%$

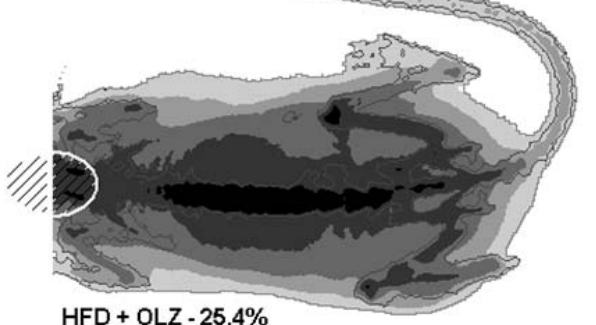




\section{Figure 4.3: HFD aggravates OLZ-induced liver injury}

Livers were stained with H\&E to demonstrate general hepatocellular morphology (panel A, top). CAE staining was used to distinguish neutrophils from other cell types (panel A, bottom). Neutrophils were counted (panel D) as described in Chapter II. Transaminase levels were measured in plasma taken at sacrifice (panels B and C). Liver weight is expressed as a percentage of total body weight (panel E). Data are means \pm S.E.M. $(n=4-8) .{ }^{a}, P<0.05$ effect of HFD; ${ }^{b}, P<0.05$ effect of OLZ as determined with two-way ANOVA and HolmŠidák multiple comparison test. 

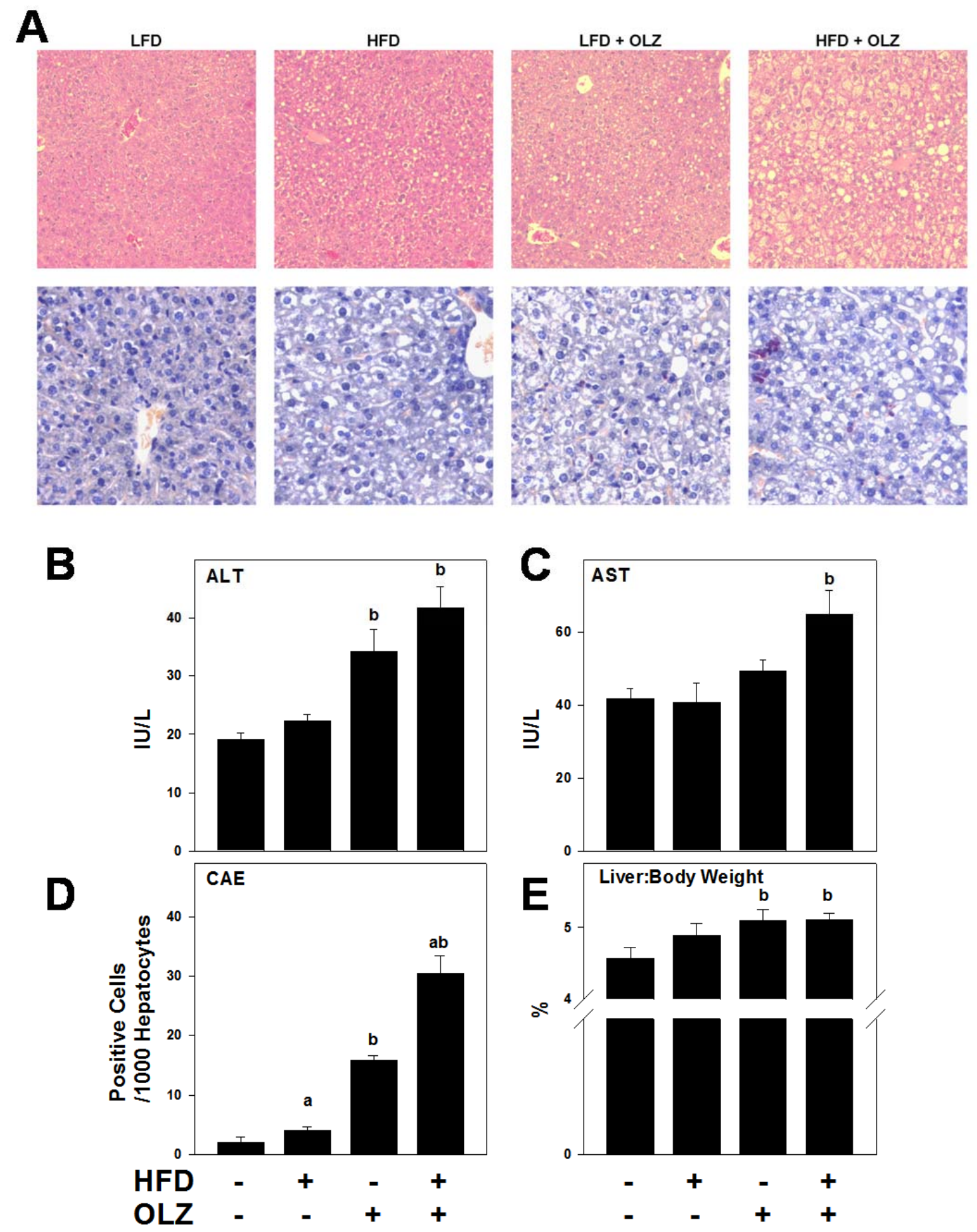
Figure 4.4: OLZ and HFD promote hepatic lipid storage

Frozen sections of liver were stained with Oil Red $\mathrm{O}$ as described in Materials and Methods (panel A, representative pictures) and staining was quantitated in all samples (panel B). Lipids (TG and NEFA) were extracted from pulverized liver samples and determined by colorimetric assay (panel C). Data are means \pm S.E.M. $(\mathrm{n}=4-8) .{ }^{\mathrm{a}}, P<0.05$ effect of HFD; ${ }^{\mathrm{b}}, P<0.05$ effect of OLZ as determined with two-way ANOVA and Holm-Šidák multiple comparison test. 

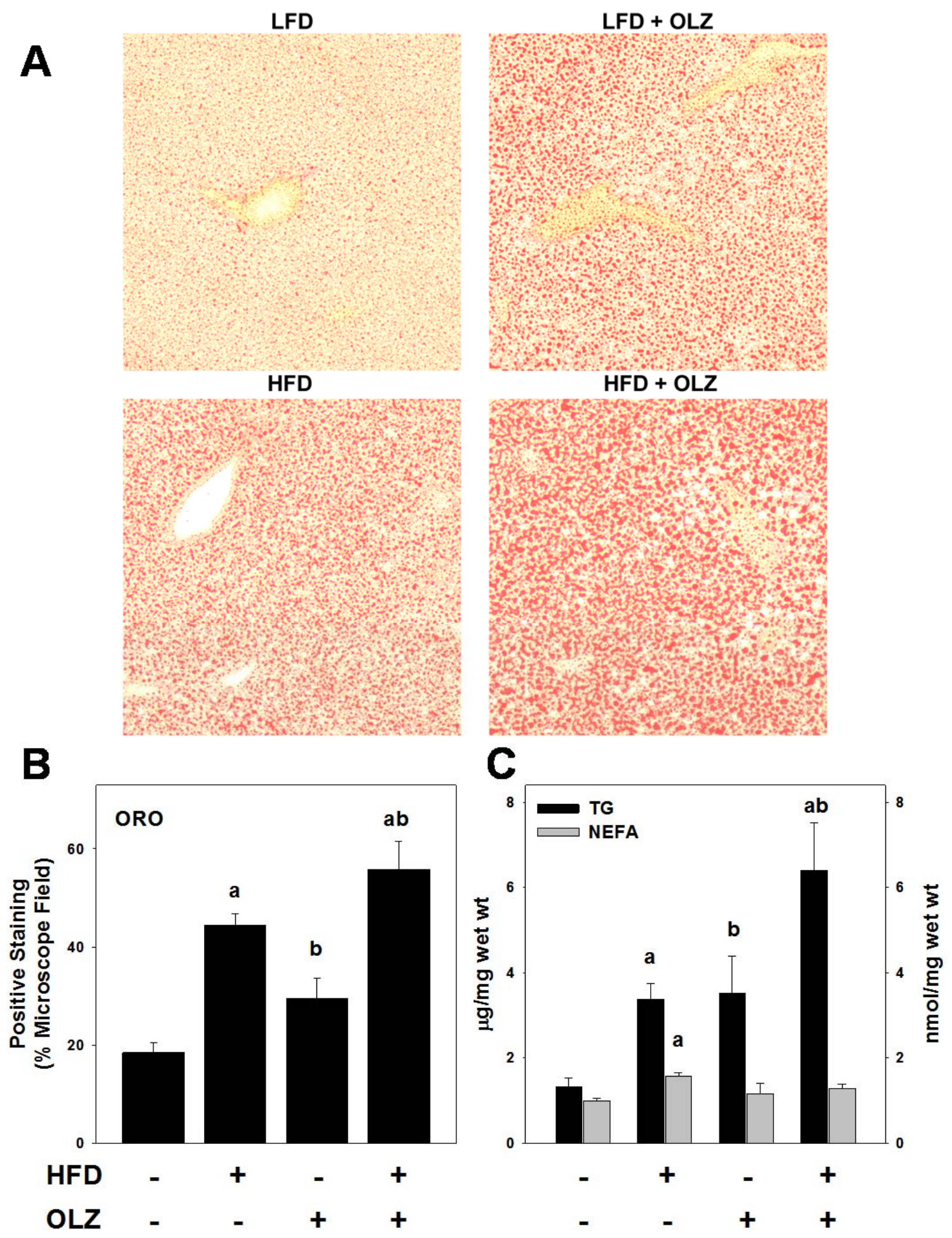
Figure 4.5: Effects of OLZ and HFD on glucose and glycogen metabolism

OGTT was performed after 25 days of HFD or LFD feeding and exposure to OLZ or vehicle (panel A). Glycogen was isolated from snap-frozen liver samples and concentrations were determined by colorimetric assay (panel B). Liver samples fixed at sacrifice were stained with Periodic acid-Schiff reagent to identify glycogen (panel C). Data are means \pm S.E.M.(n=4-8). ${ }^{a}, P<0.05$ effect of HFD; ${ }^{b}, P<0.05$ effect of OLZ as determined with two-way ANOVA and HolmŠidák multiple comparison test. 

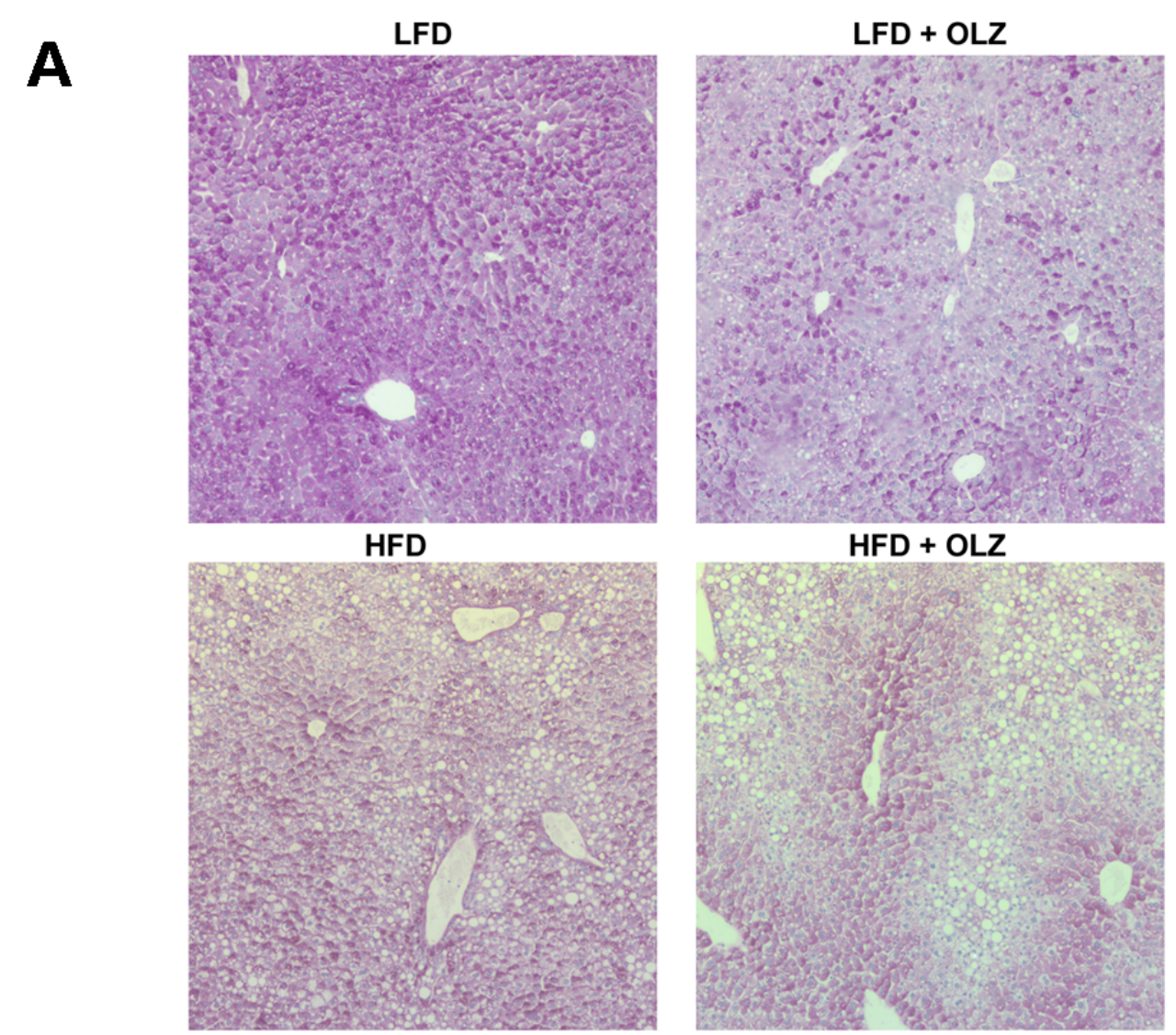

B

C
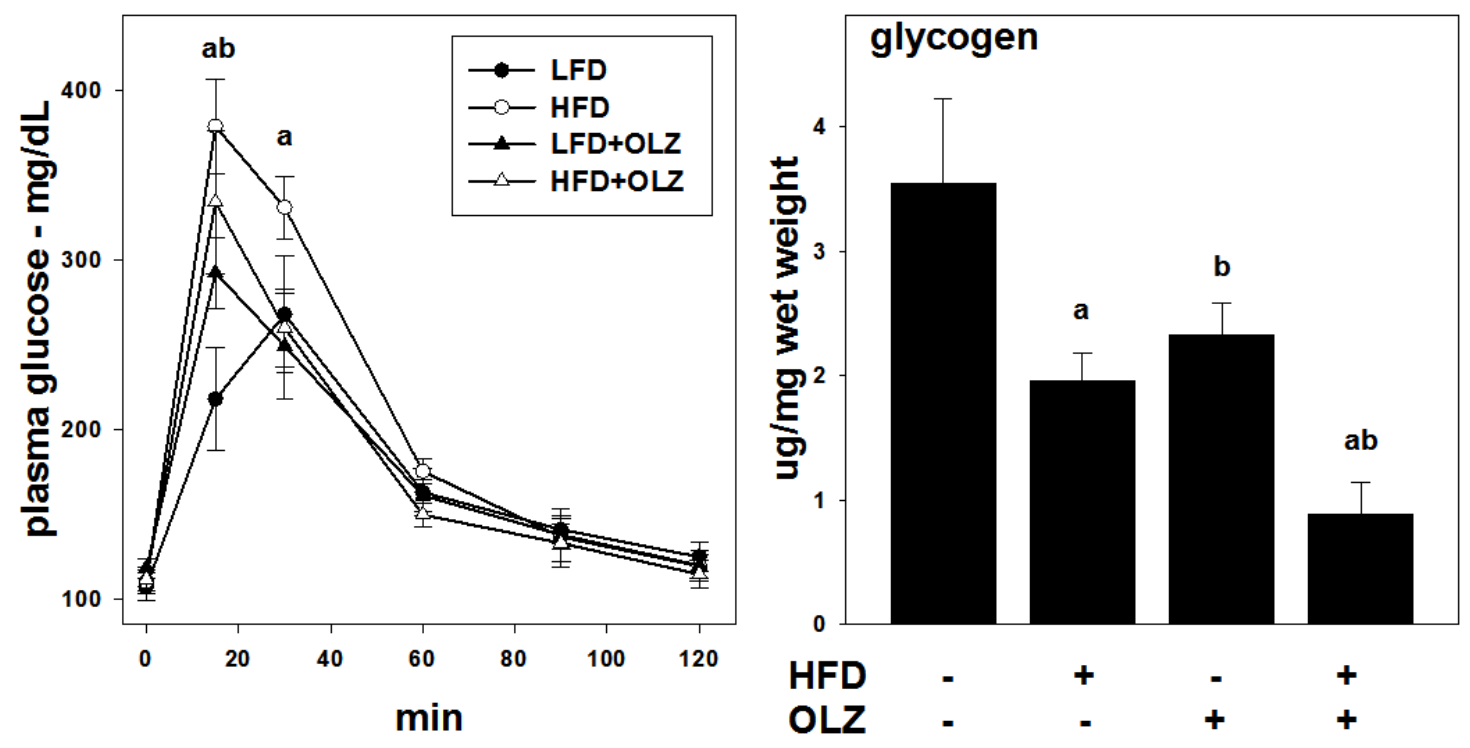


\section{Figure 4.6: Effects of OLZ on apoptosis and lipid peroxidation}

Frozen liver sections were stained using a modified TUNEL assay (panel A, top row). Cells were counted (panel B) as described in the Histology section of Chapter II, Experimental Procedures. Formalin-fixed liver sections were used for immunohistochemical detection of 4-HNE adducts (panel A, bottom row). Extent of staining (panel C) was quantitated as described in Experimental Procedures. Data are means \pm S.E.M.(n=4-8). ${ }^{a}, P<0.05$ effect of HFD; ${ }^{b}, P$ $<0.05$ effect of OLZ as determined with two-way ANOVA and Holm-Šidák multiple comparison test. 

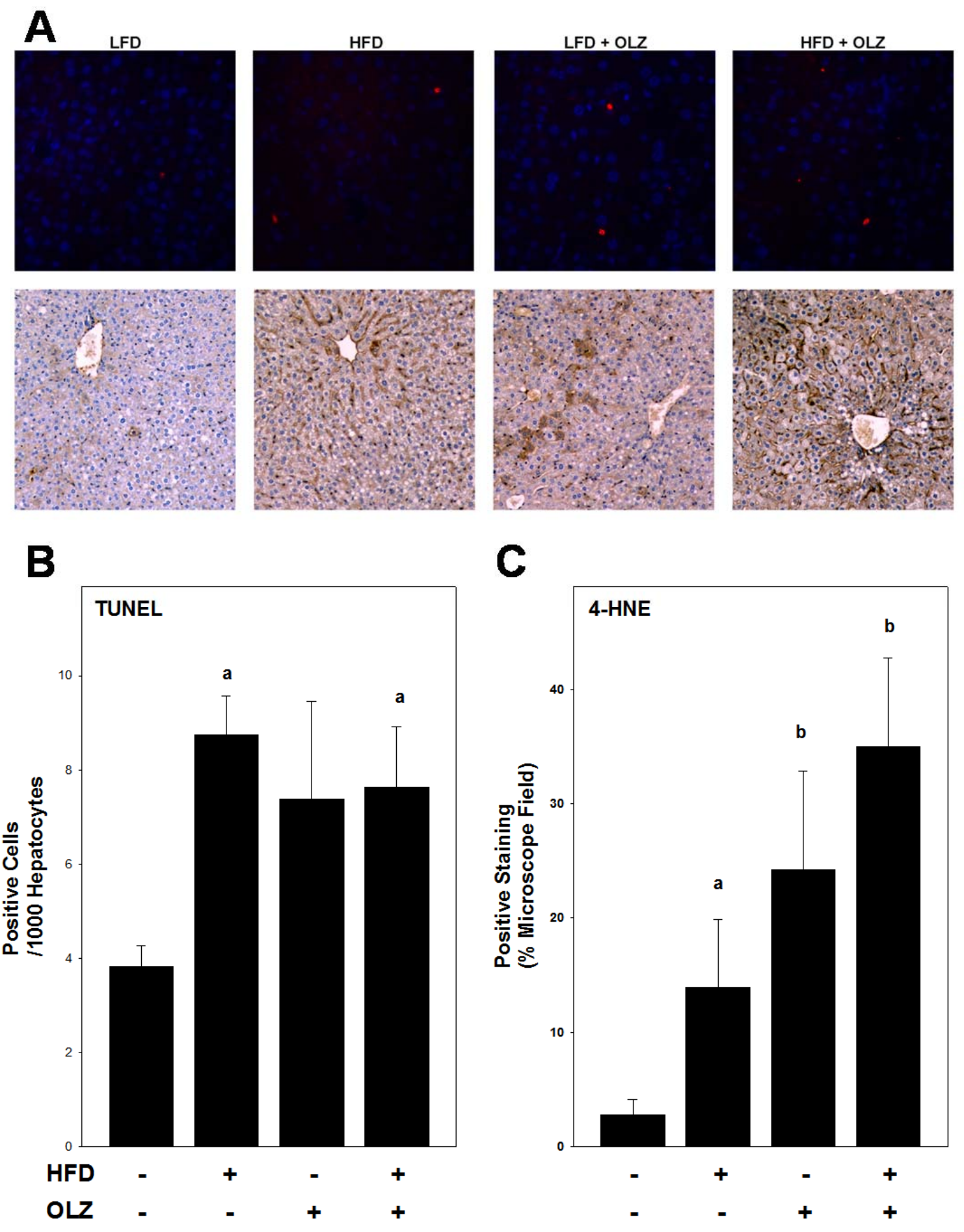


\section{CHAPTER V}

\section{PROTECTION FROM OLANZAPINE-INDUCED LIVER INJURY THROUGH ACTIVATION OF THE NRF2 PATHWAY}

\section{A. Introduction}

Sulforaphane (SFN), an isothiocyanate phytochemical, is known to confer antioxidant protection in vivo. Rather than directly reacting with oxidants, however, SFN works by inducing Nrf2, a transcription factor that binds to the promoter regions of several known antioxidant genes and enhances detoxification (diagram, Figure 5.1). The route from SFN to Nrf2 is not entirely established. In some studies, SFN's isothiocyanate functional group is shown to participate as a Michael acceptor in the nucleophilic reaction with cysteine residues on Keap1. ${ }^{129}$ If Nrf2 remains bound to Keap1, it remains by definition in the cytosol, where it does not influence gene transcription and will eventually be targeted for degradation by the ubiquitin ligase Cul3. ${ }^{130}$ In addition to releasing Nrf2 from Keap1, SFN may raise overall expression of Nrf2. Previous experiments from this laboratory have shown that SFN increases Nrf2 abundance in both nuclear and cytoplasmic subcellular compartments, which may account for the enhanced antioxidant protection observed by others.

Both obesity and OLZ-induced dysmetabolism are mediated in part by oxidative stress. This implies that either condition may benefit from antioxidant 
intervention, e.g., SFN treatment. It may then be possible to prevent hepatic injury from concurrent obesity and OLZ administration by pretreatment with SFN. The purpose of the current study was to test the protective effect of SFN against HFD- and OLZ-induced hepatic injury.

\section{B. Experimental procedures}

\section{Animals and treatments}

Mice were administered HFD and OLZ for four weeks. Some mice were also administered SFN as described in Chapter II.

\section{Biochemical analyses and histology}

ALT and AST were measured as previously described. H\&E, TUNEL, and 4-HNE staining were performed as described in Chapter II.

\section{Glycogen determinations}

Glycogen was determined in aliquots of frozen liver as described in Chapter II.

\section{Lipid determinations}

Lipids were extracted and analyzed as discussed in Chapter II.

\section{Results}

1. SFN prevents steatosis and liver injury caused by co-administration of HFD and OLZ Histological changes were assessed by H\&E staining (Figure 5.2, panel A). HFD and OLZ, as expected, caused hepatic steatosis and mild inflammation. 
SFN treatment decreased fat accumulation in liver (panel A) in addition to decreasing neutrophil infiltration/inflammation (panel A, inset). ALT and AST, commonly used as markers of liver injury, decreased in parallel with the changes in histology (panel B).

\section{SFN improves lipid and carbohydrate metabolism in mice}

\section{administered HFD and OLZ}

SFN blunted the characteristic triglyceride (TG) accumulation caused by HFD and OLZ (Figure 5.2, panel B). SFN also rescued non-esterified fatty acid (NEFA) levels which are paradoxically low during OLZ administration, ${ }^{70,104}$ and increased hepatic glycogen storage, which is synergistically impaired by HFD and OLZ (Figure 5.2, panel B and Figure 5.3, panel A).

\section{SFN does not affect apoptosis with HFD and OLZ, but greatly} decreases lipid peroxidation

Adducts of 4-HNE, which amassed in large quantity after HFD and OLZ exposure (see Chapter IV, Figure 4.4, panel A), appeared to nearly absent from the tissue sections of SFN-treated mice (Figure 5.3, panel B) .The visually apparent attenuation in staining was quantitated and determined to be a $>20$-fold decrease in 4-HNE binding. Apoptosis (as determined by TUNEL staining) was unaffected by SFN (Figure 5.3, panel C).

\section{Discussion}

Obesity and antipsychotic-induced dysmetabolism may both be mediated by oxidative stress (see Chapter I for general concepts of oxidative stress). 
Whereas in obesity it is the reactive metabolites of sugars and lipids that may cause oxidative stress, ${ }^{131,132}$ atypical antipsychotics may reduce expression of antioxidant-encoding genes ${ }^{133}$ in addition to generating reactive metabolites. ${ }^{134}$ Antipsychotics can also be considered secondary contributors to oxidative stress in that they promote obesity. Although the exact presentation of oxidative stress in OLZ metabolism is not certain, it may be possible to prevent or treat the weight-related side effects of OLZ using antioxidants.

In vitro, direct-acting antioxidants such as resveratrol consistently display the ability to neutralize reactive oxygen species (ROS) and reactive drug metabolites. ${ }^{135}$ In vivo, however, the success of antioxidant interventions has been difficult to replicate. ${ }^{68}$ The pharmacokinetic and pharmacodynamic limitations of direct-acting antioxidants like resveratrol may explain the incongruous in vitro and in vivo experimental outcomes. Direct antioxidants are reactive species themselves, making it difficult to ensure that, in the intact organism, direct antioxidants reach the desired site of action before reacting with other biological molecules. Agents like resveratrol and vitamin $\mathrm{C}$ are also prone to redox cycling, meaning that they will readily act as both a reductant and an oxidant in a complex living system. ${ }^{69}$ This means that in practice, high doses of direct antioxidants can contribute to the same problems that they are intended to solve.

Indirect antioxidants do not usually participate in redox reactions and therefore offer an alternative approach to manage oxidative stress. SFN is repeatedly shown to enhance Nrf2 accumulation, rather than interact directly with 
ROS. ${ }^{69}$ Here also, the indirect antioxidant sulforaphane was used successfully to prevent oxidative stress in a model of chronic high-fat diet and olanzapine administration. No exacerbation of oxidative stress was noted. The action of intrinsic cellular defense mechanisms by SFN therefore exemplifies an effective strategy to offset the side effects of antipsychotic treatment. It should be considered as a therapeutic adjunct in future studies of patients prescribed OLZ. 


\section{Figure 5.1: Simplified model of Nrf2 induction by SFN}

SFN's electrophilic side chain alkylates cysteine residues on Keap1. The conformational change induced in Keap1 allows the release of its binding partner, Nrf2. Unbound Nrf2 translocates to the nucleus and binds to antioxidant response elements in DNA. See Discussion in section A of Chapter VI for additional explanation of this mechanism. 


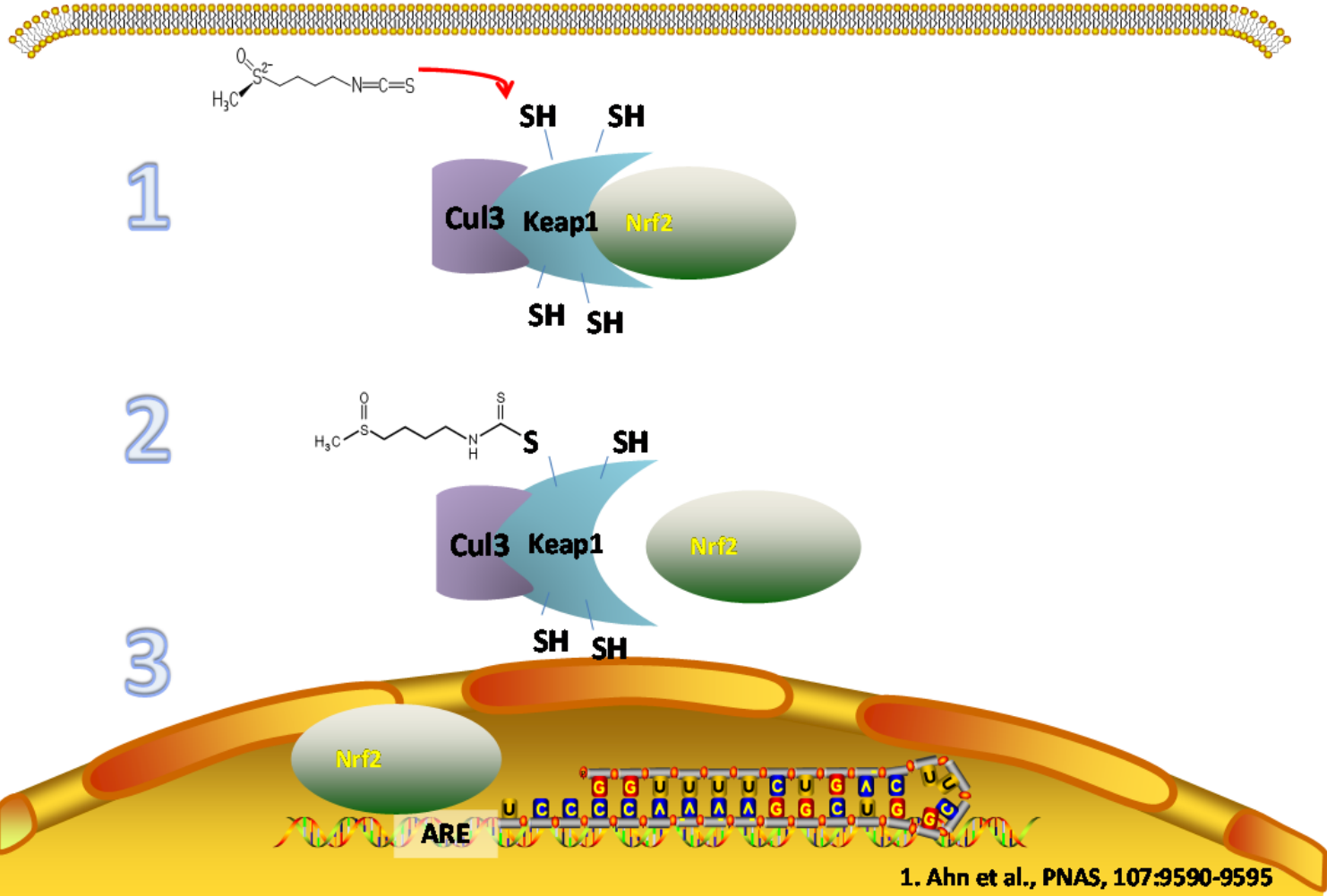


Figure 5.2: Influence of SFN on the metabolic outcomes of HFD and OLZ co-administration

Panel A: H\&E staining is shown at $100 x$ and $400 x$ (inset). Panel B: Weight and other parameters were measured after HFD and OLZ treatment in the presence or absence of SFN. Values for the SFN treatment group are expressed as fold change vs. HFD and OLZ in the absence of SFN. 
A
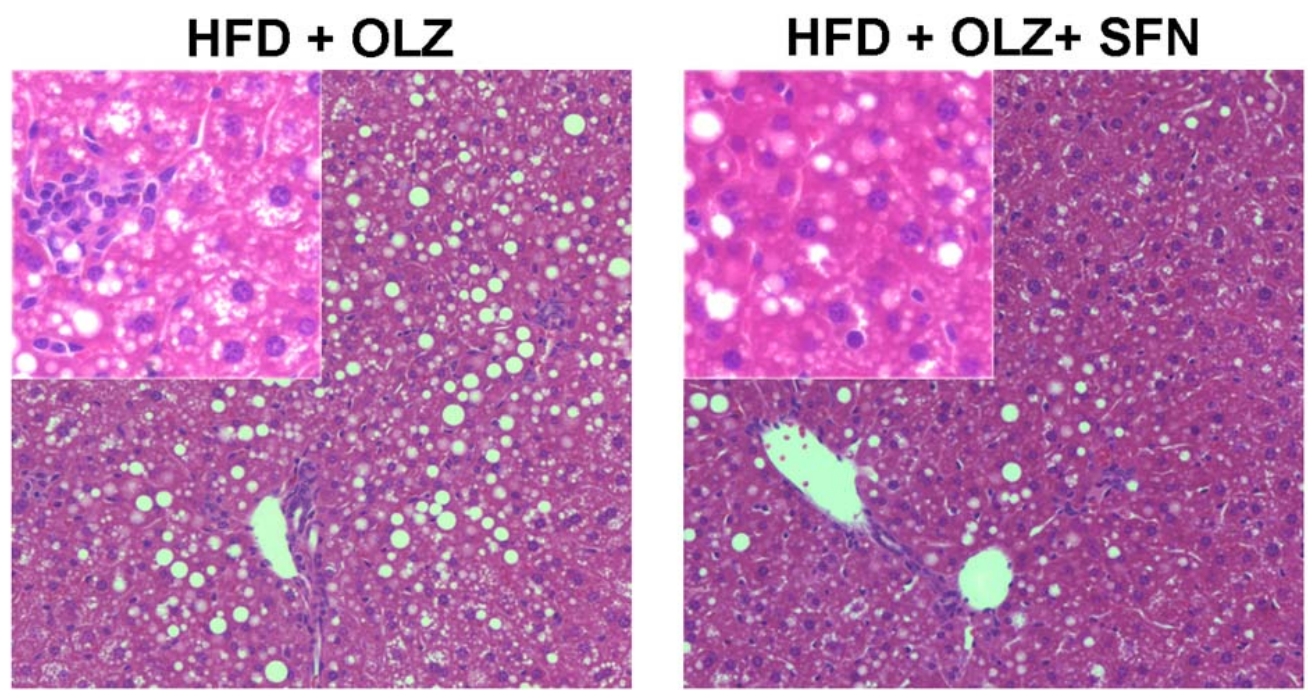

B

HFD + OLZ + SFN vs HFD + OLZ

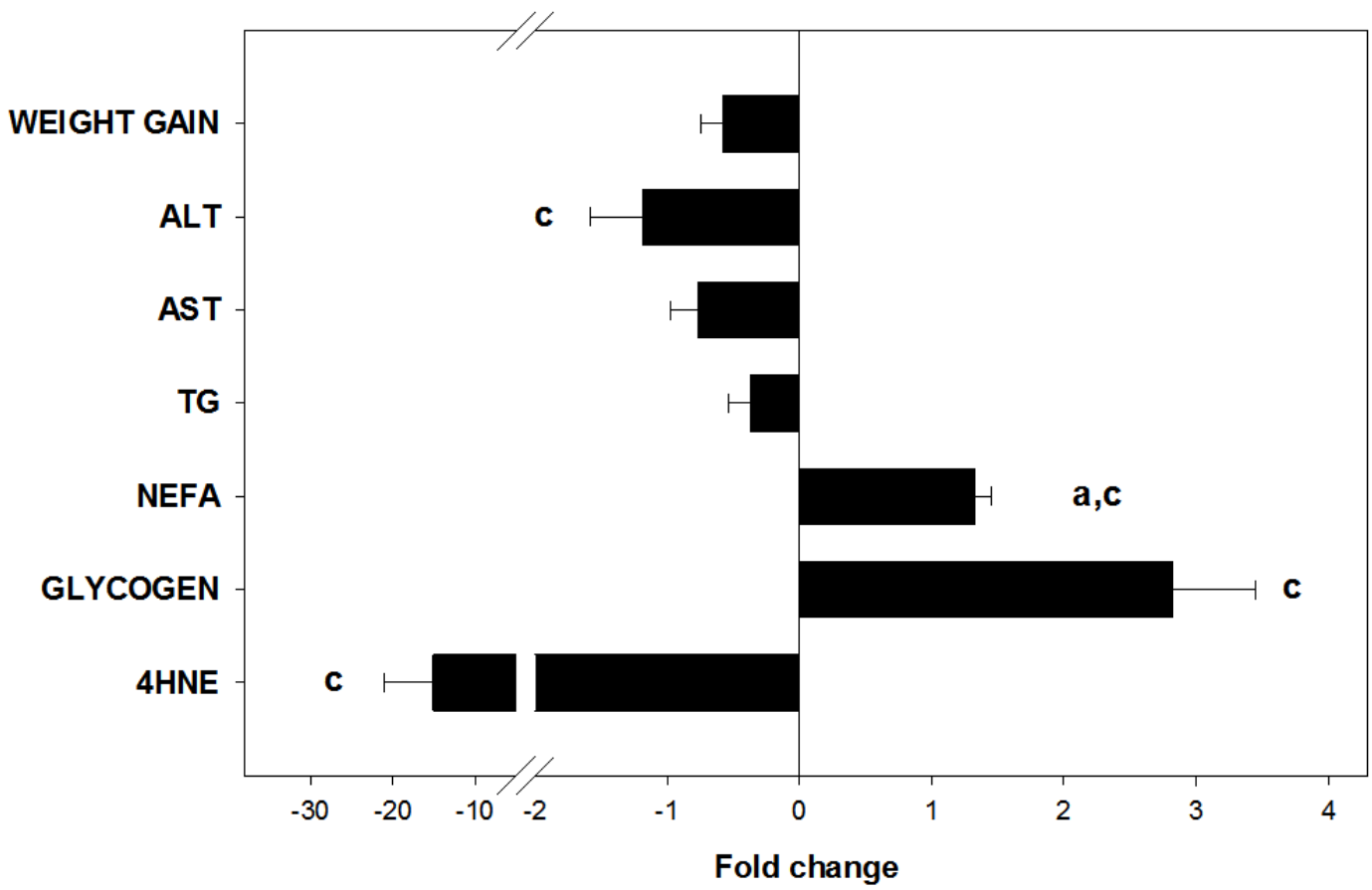




\section{Figure 5.3: Histological indices of protection from HFD and OLZ by SFN}

Livers sections from animals given HFD and OLZ were compared with liver sections from animals who were additionally co-administered SFN. Staining for glycogen (panel A), lipid peroxidation (panel B), and apoptosis (panel C) was performed as described in Experimental Procedures, Chapter II. 

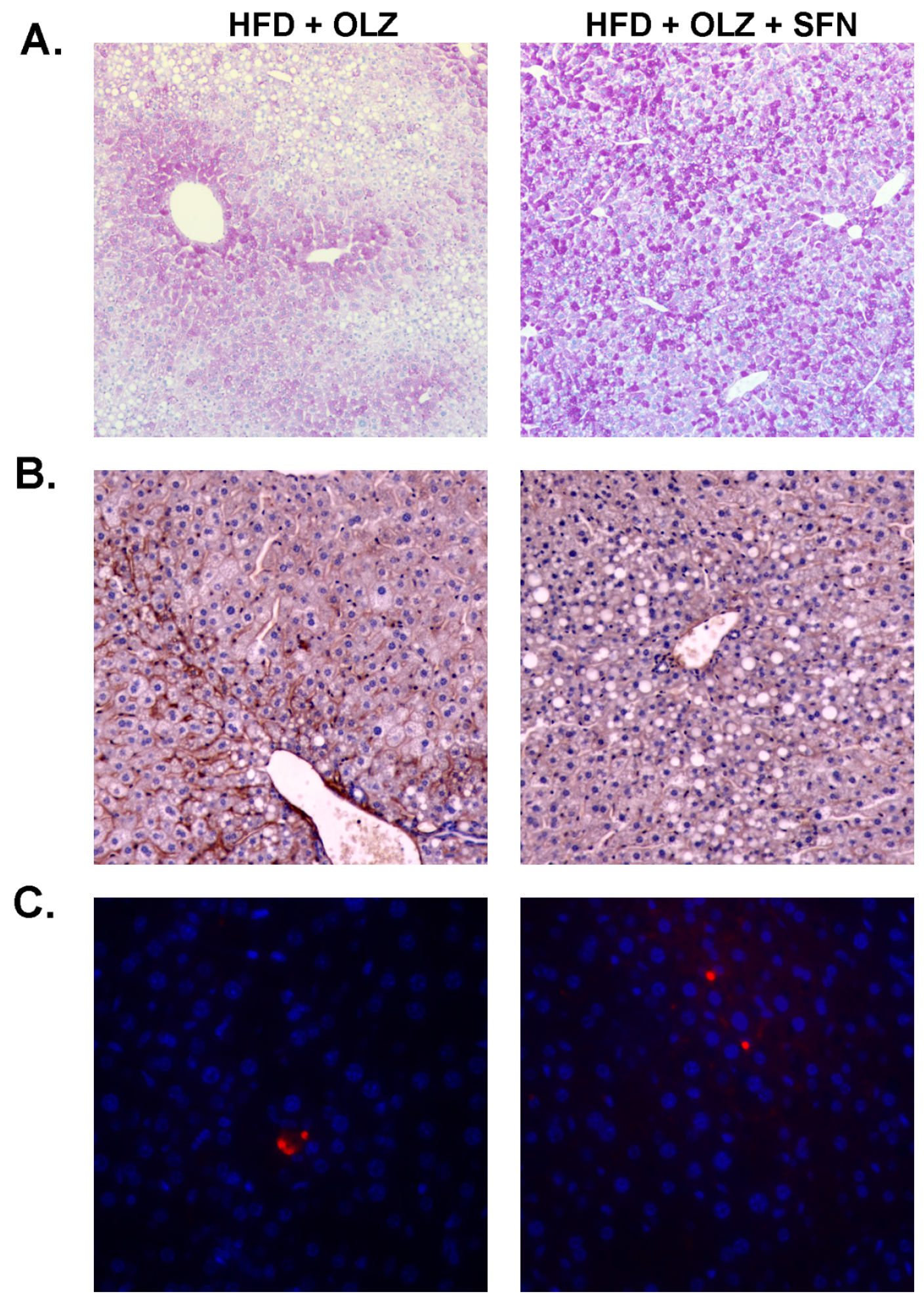


\section{CHAPTER VI \\ DISCUSSION AND CONCLUSIONS}

\section{A. Biochemical pathways involved in NAFLD and/or OLZ toxicity and potential pharmacological interventions}

\section{AMPK}

One of the major regulators of energy homeostasis in mammalian cells is the $5^{\prime}$ adenosine monophosphate-activated protein kinase (AMPK). ${ }^{113}$ The ratio of AMP:ATP is roughly proportional to cellular nutrient availability; ${ }^{136}$ therefore, AMPK's ability to respond to changes in the AMP:ATP ratio makes it an important coordinator of various metabolic functions. When AMPK's $\alpha$ subunit is phosphorylated at threonine-172, the kinase is activated and promotes ATPgenerating catabolic processes, e.g., fatty acid oxidation. It is through increased catabolism, in fact, that the AMPK-activating drug metformin is thought to reverse fatty liver and glucose intolerance. ${ }^{137}$

Along with its well-established roles in energy homeostasis, research has also demonstrated a link between AMPK activation and atypical antipsychotics such as OLZ. AMPK activation in the hypothalamus is frequently reported after OLZ administration, leading many researchers to consider its mechanistic relevance to treatment-emergent obesity. ${ }^{138,139}$ AMPK activation has also been 
shown in PC12 cells (i.e., non-neuronal cells) after OLZ exposure, demonstrating that AMPK activation with OLZ is not restricted to the CNS. ${ }^{140}$ Meltzer ${ }^{141}$ and others have suggested that AMPK activation may be inducing the metabolic side effects of OLZ. It remains unclear, though, why AMPK activation by OLZ would promote symptoms of the metabolic syndrome when AMPK activation by metformin has clear antidiabetic effects. ${ }^{142}$ The moderate success of metformin in treating OLZ-induced diabetes has only further confounded the search for answers. ${ }^{143}$

\section{2. $\mathrm{mTOR}$}

The mammalian target of rapamycin (mTOR) is a widely-expressed serine-threonine kinase (see Tsang ${ }^{109}$ for review). It is found as part of two functional complexes, mTORC1 and mTORC2, both of which are major mediators of cell growth and survival. mTOR may be seen as a counterpart to AMPK: while AMPK is activated under conditions of nutrient depletion, mTOR is typically activated during times of adequate or surplus nutrient availability. ${ }^{144}$ The functions of mTOR, then, are mostly anabolic, including RNA transcription ${ }^{145}$ and adipogenesis. ${ }^{146}$ While some degree of mTOR activation is necessary, excessive activation of mTOR is implicated in obesity and diabetes. Constitutive mTOR activation in vitro has been shown to impair insulin signaling. ${ }^{147}$ In further support of mTOR's hypothesized role in metabolic disorders, a clinical study published just earlier this year reported that mTORC1 signaling is activated in placental samples taken from obese mothers. ${ }^{148}$ 
There is no clear link between mTOR and atypical antipsychotic drug actions; however, they are connected by a wealth of circumstantial evidence. Among its numerous other functions the mTOR pathway regulates memory and synaptic plasticity. ${ }^{149}$ Deletion of Rictor, the mTORC2 binding partner, in a mouse model induces schizophrenia-like behaviors and abnormal neurotransmission. ${ }^{150}$ Rapamycin, the most extensively studied mTOR inhibitor, has been suggested as a potential treatment for mood disorders; ${ }^{151}$ at the same time, ketamine, a known mTOR inducer, demonstrably improved bipolar disorder in a handful of small clinical trials. ${ }^{152,153}$ There are still huge gaps in our knowledge of mTOR and neurological dysfunction. At present, there are no FDA-approved treatments for mental illness or obesity that directly target mTOR.

\section{Nrf2}

NF-E2-related factor 2 (Nrf2) is a basic leucine zipper transcription factor universally expressed in the cytosol of eukaryotic cells. ${ }^{154,155}$ In the presence of electrophiles or reactive oxygen species, Nrf2 is released from its repressor protein Keap1 and the ubiquitin ligase Cul3 ${ }^{156,157}$ Upon its release from Keap1 and Cul3, Nrf2 moves to the nucleus, where it can then bind to response elements in DNA ${ }^{158}$ and influence antioxidant gene transcription. Because reactive oxygen species are proposed culprits in many disease states, ${ }^{53-56} \mathrm{Nrf2-}$ inducing chemicals are being explored as therapeutic agents. ${ }^{69}$ Nrf2's ability to upregulate expression of cytoprotective genes makes it a potentially important target for drug development. 
Nrf2 and mTOR are not usually considered as parts of a common pathway. However, these pathways do intersect, most notably at the level of

insulin signaling. ${ }^{159}$ The actions of rapamycin and the experimental Nrf2 inducer CDDO-Im also suggest cross-talk between the pathways: Nrf2-mutated lung cancer cells are responsive to rapamycin, ${ }^{160}$ while CDDO-Im inhibits insulinmediated mTOR phosphorylation. ${ }^{161}$

\section{B. Major findings of this dissertation}

It has been stated several times throughout this work that OLZ is an effective drug for treating psychiatric illnesses including schizophrenia and bipolar disorder. ${ }^{162,163}$ The benefits of OLZ are countermanded by side effects such as weight gain, glucose intolerance and dyslipidemia which impact not only psychiatric treatment compliance but also increase the health risks to patients. An improved understanding of OLZ actions in the peripheral tissues may therefore identify new approaches to increase the long-term safety and utility of this drug. Experiments in Chapter III were performed to reveal and characterize the mechanism(s) of OLZ-induced toxicity. In Chapter IV, the experiments of the previous chapter were expanded to ascertain possible interactions of OLZ and the typical Western diet. The experiments in Chapter V tested SFN as an adjunct to OLZ administration. Specific findings from each of these chapters are discussed in the next paragraphs.

\section{OLZ simultaneously activates AMPK and mTOR}

As has been observed previously, OLZ administration increased body weight and adiposity in mice (Figure 3.2) in the absence of a high-fat diet or a 
detectable increase in food consumption. These data suggest that OLZ administration may directly alter energy usage in vivo. Previous experiments from other laboratories have shown that OLZ administration dysregulates carbohydrate and lipid metabolism (see Coccurrello ${ }^{94}$ for review). Indeed, here, OLZ administration caused a complex phenotypic alteration in hepatic carbohydrate metabolism. OLZ administration increased glycolysis without an apparent increase in mitochondrial respiration (Figures 3.4 and 3.6) and favored glycogen depletion (Figures 3.4 and 3.7). OLZ administration also caused a slight but significant glucose intolerance (Figure 3.7) and increased postprandial plasma glucose (Table 3.1). A shift to an elevated, but incomplete (i.e., anaerobic), glycolysis would favor ATP generation from alternate sources, such as lipid oxidation. In support of the hypothesized shift to glycolysis, OLZ administration increases plasma lactic acid levels in humans. ${ }^{94}$ Furthermore, Albaugh et al. ${ }^{104}$ demonstrated that OLZ administration lowers the respiratory exchange ratio (RER) in rats, indicative of a preferential shift to NEFA over carbohydrates as fuel. These results are in line with the observation here that hepatic and plasma triglycerides were elevated by OLZ administration (Figure 3.3 and Table 3.1), without a commensurate increase in hepatic NEFA (Figure 3.3).

Within the cell, carbohydrate and lipid metabolism are usually under the tight control of the protein kinases AMPK and mTOR. Both AMPK and mTOR are known to act as "sensors" of cellular energy status and help to maintain homeostasis. ${ }^{113,164}$ In general, the downstream effects of AMPK activation are considered catabolic and favor ATP generation during energy depletion. 
Glycolysis, for example, is enhanced by AMPK. Signaling downstream of AMPK also inhibits ATP-consuming processes. ${ }^{165}$ In contrast to AMPK, mTOR is activated during times of high nutrient availability and favors storage of excess nutrients (e.g., as triglycerides). Activation of the mTOR pathway promotes ATPconsuming processes such as protein and lipid synthesis through its downstream targets p70S6K and 4EBP1. Earlier studies have demonstrated that AMPK is activated in the CNS by OLZ administration. ${ }^{140}$ Furthermore, OLZ increases p70S6K and 4EBP1 phosphorylation in cultured hepatocytes, indicative of mTOR activation. ${ }^{166}$ The experiments here demonstrate, for the first time, that OLZ in vivo concomitantly activates AMPK and mTOR pathways in the liver. AMPK and mTOR are generally differentially activated and mediate opposing cellular functions. ${ }^{144}$ The outcomes of OLZ administration on hepatic metabolism reflect, in part, these contradictory inputs. These data suggest that treatment with OLZ results in a pseudo-fasted state wherein metabolic resources are abundant, but cannot be efficiently used. The reason for OLZ's concurrent activation of AMPK and mTOR is not immediately clear. Should OLZ indeed induce a pseudo-fasted state, the activation of AMPK may be, in principle, via the classical accumulation of AMP.

\section{OLZ modulates intracellular amino acid transport, which may affect} energy homeostasis

OLZ is reported elsewhere to modulate glutamine, glutamate and BCAA concentrations in brain and periphery, as well as key genes in glutamate metabolism. ${ }^{42,101,108,167-169}$ The functional therapeutic importance of these amino 
acid changes is uncertain, but may be related to maintenance of the so-called "glutamate-glutamine cycle."170-172 This is a model which proposes that excitatory neurotransmission is carefully maintained through the coordinated transport of glutamate and glutamine. The cycle is neither closed nor stoichiometric ${ }^{173}$ but nonetheless finely synchronizes amino acid concentrations between neuronal and non-neuronal compartments. In the experiments presented here, OLZ administration modulated glutamate, glutamine, and leucine flux, while pathway analysis pointed to increases in the amino acid transporters LAT1, SNAT2, and EAAT3, among others. The actions of OLZ seen here and in others' work are consistent with the glutamatergic model of mental illness, which proposes that psychotic disorders are (at least partly) disorders of excitatory amino acid metabolism. ${ }^{123}$ Notably, hepatic mTOR activation is also mediated by these same fluctuations in amino acid metabolism. ${ }^{97,106,116,174-176}$ Meta-analyses have demonstrated that targeting AMPK with metformin confers a significant, albeit small, protective effect against some of the metabolic effects of OLZ. ${ }^{115}$ However, targeting mTOR overactivation may be a more successful approach.

\section{HFD aggravates liver injury caused by OLZ}

Another important aspect of this work is that the combination of HFD and OLZ administration significantly increased indices of liver injury (Figure 4.3) in mice. Elevation of liver enzymes is described as a common reaction with OLZ administration, resulting in drug discontinuation. ${ }^{177}$ The pathologic changes in liver due to OLZ alone (Table 3.1 and Figure 4.3) are analogous to those caused by HFD. However, in many of the experiments performed here, injury from HFD 
and OLZ together was greater than the injury resulting from either factor on its own, possibly indicating that these factors mediate toxicity through separate, distinct mechanisms. NAFLD is a spectrum of liver diseases, ranging from the relatively benign simple steatosis to hepatocellular carcinoma. ${ }^{27}$ Although the prevalence of simple steatosis in individuals at risk for NAFLD can be very high, ${ }^{46}$ the prevalence of $\mathrm{NASH}$ is much lower in this population $(\sim 40 \%) .{ }^{46}$ These factors emphasize that the risk for developing more severe stages of NAFLD (i.e., NASH and beyond) is not based solely on obesity, but is rather mitigated by other factors. ${ }^{47,48}$ It is likely that OLZ treatment could be such a factor that enhances the progression of NAFLD.

\section{SFN counteracts oxidative stress caused by HFD and OLZ}

Much of the research regarding Nrf2 has focused on the pathway's function in mitigating oxidative stress. ${ }^{178}$ Though antioxidant defense is a primary feature of Nrf2 pathway induction, recent investigation shows that Nrf2 also exerts regulatory effects on lipid metabolism. ${ }^{179} 180$ Moreover, because oxidative stress and altered lipid metabolism are both correlated with the side effects of OLZ, Nrf2 induction (using SFN) was tested as a method of minimizing these side effects.

SFN, as predicted, offset many of the side effects of OLZ without causing apparent injury to the mice (Figure 5.2). The most significant improvements seen were in weight, adiposity, and lipid peroxidation. The favorable results with SFN suggest that Nrf2 induction may be used to decrease the incidence of additional medical complaints that can be caused by OLZ. Consequently, SFN 
administration might also encourage patient adherence with psychiatric treatment regimens.

\section{Strengths and weaknesses of this dissertation}

\section{Strengths}

There are many strengths of this dissertation. One such strength is that it identifies a new mechanism in the development of metabolic dysregulation caused by OLZ. It also offers additional evidence for the worsening of liver disease during antipsychotic administration and suggests a possible strategy for prevention. The research presented in this text thus advances the ongoing search for better-tolerated pharmacological treatments for mental illness.

Another strength of this dissertation is the use of whole animal models. Most of the experiments described here were performed in vivo, except where to do so would be impractical or impossible (e.g., measuring oxygen consumption in real time with multiple doses of OLZ). Obesity and mental illness are both complex phenomena, not limited to one particular organ or cell type. Also, like most psychiatric medications, OLZ is widely systemically distributed, as this is the most practical method of delivering the drug to the CNS. ${ }^{181}$ As such it is preferable to study the effects of OLZ in a complex, dynamic system such as an intact organism.

\section{Weaknesses}

The use of animal models, while a strength, is also a potential weakness. Although in vivo research is preferred for many pharmacological and metabolic 
studies, no animal model can completely recapitulate the human condition. Apart from obvious differences in sleeping, eating, and social interactions, there is also no rodent model that exactly represents human mental illness ${ }^{182}$ or non-alcoholic liver disease progression from steatosis to $\mathrm{NASH}^{61}$

Another weakness of this study is that all conditions are not adequately represented in the SFN experiment. The combination of OLZ and low-fat/control diet was not tested with SFN, nor was SFN administered in any diet group in the absence of OLZ. Additionally, increased drug efflux is a known consequence of Nrf2 induction. ${ }^{183}$ More research will be needed to determine whether or not SFN is promoting OLZ removal and perhaps jeopardizing the therapeutic potential of OLZ.

\section{Future directions}

Research answers scientific questions, but just as often it creates new questions. The following are future experiments that logically proceed from the findings of this dissertation:

\section{What are the effects of combined HFD and OLZ exposure on AMPK and mTOR expression?}

Because the experiments described in Chapters IV and V were largely concerned with pathology and not biochemical mechanism of development, mTOR, AMPK, and related proteins in these signaling pathways were not measured in animals treated with HFD and/or SFN. Obesity and high-fat diet by

themselves reproducibly activate mTOR signaling; ${ }^{184}$ however, the effects of 
concomitant HFD and OLZ on mTOR regulation have not been explored.

Likewise, the existence of a relationship between mTOR and Nrf2 is not firmly established; it would therefore be interesting to determine how mTOR signaling and expression are mediated with SFN alone, SFN in combination with HFD, and SFN with concurrent HFD and OLZ.

\section{Does SFN influence absorption, distribution, metabolism, or} excretion of OLZ?

In the Weaknesses section of this chapter, it was mentioned that metabolic response to SFN was not observed in all possible conditions, i.e., with low-fat control diet or without OLZ. While it is clear that SFN prevented weight gain and liver injury in the HFD + chronic OLZ model, it is not certain how SFN did this. SFN is widely shown to prevent oxidative stress and is also understood to mediate lipid metabolism, but neither function of SFN was conclusively determined in any of the preceding experiments. Perhaps even more importantly, it was not determined how SFN co-administration affects OLZ disposition. For example, glucuronidation is recognized to be increased by SFN administration, ${ }^{185}$ which may suggest that SFN worked in this model simply by increasing OLZ excretion and removal. As Bever and Perry ${ }^{1}$ noted in their review, OLZ is metabolized by many known mechanisms, so it is unlikely that a change in any one pathway would affect total clearance of OLZ. Still, excessive clearance of OLZ should be ruled out in future experiments.

3. How does OLZ behave in other peripheral locations, such as adipose tissue? 
The approved uses of OLZ are psychiatric disorders. The most common off-label uses for OLZ are mental illness-related as well. Naturally, much of the existing literature is devoted to the study of OLZ in central nervous system tissue. In order to gain a more complete understanding of how OLZ works, its effects should be more extensively studied in non-neuronal regions, both in the intact organism and in cell culture. A prime candidate for future study is adipose tissue which, despite its known role as an arbiter of leptin secretion and CNS recognition of satiety, ${ }^{186}$ remains poorly investigated. Skeletal muscle is also overlooked in antipsychotic research, even though myopathy is sometimes reported during OLZ administration to human patients, ${ }^{187}$ and should also be thoroughly investigated. 


\section{REFERENCES}

1. Bever, K. A.; Perry, P. J. Olanzapine: a serotonin-dopamine-receptor antagonist for antipsychotic therapy. Am J Health Syst Pharm. 1998, 55 (10), 1003-1016.

2. Borison, R. L. Clinical efficacy of serotonin-dopamine antagonists relative to classic neuroleptics. J Clin Psychopharmacol. 1995, 15 (1 Suppl 1), 24S-29S.

3. Bymaster, F. P.; Calligaro, D. O.; Falcone, J. F.; Marsh, R. D.; Moore, N. A.; Tye, N. C.; Seeman, P.; Wong, D. T. Radioreceptor binding profile of the atypical antipsychotic olanzapine.

Neuropsychopharmacology. 1996, 14 (2), 87-96.

4. Moore, N. A.; Tye, N. C.; Axton, M. S.; Risius, F. C. The behavioral pharmacology of olanzapine, a novel "atypical" antipsychotic agent. J Pharmacol Exp. Ther. 1992, 262 (2), 545-551.

5. Edlinger, M.; Baumgartner, S.; Eltanaihi-Furtmuller, N.; Hummer, M.; Fleischhacker, W. Switching between second-generation antipsychotics: why and how? CNS Drugs. 2005, 19 (1), 27-42.

6. Tran, P. V.; Hamilton, S. H.; Kuntz, A. J.; Potvin, J. H.; Andersen, S. W.; Beasley, C., Jr.; Tollefson, G. D. Double-blind comparison of olanzapine versus risperidone in the treatment of schizophrenia and other psychotic disorders. J Clin Psychopharmacol. 1997, 17 (5), 407-418.

7. Stubner, S.; Grohmann, R.; Engel, R.; Bandelow, B.; Ludwig, W. D.; Wagner, G.; Muller-Oerlinghausen, B.; Moller, H. J.; Hippius, H.; Ruther, E. Blood dyscrasias induced by psychotropic drugs. Pharmacopsychiatry. 2004, 37 Suppl 1:S70-8., S70-S78.

8. Maher, A. R.; Theodore, G. Summary of the comparative effectiveness review on off-label use of atypical antipsychotics. J Manag Care Pharm. 2012, 18 (5 Suppl B), S1-S20. 
9. Fountoulakis, K. N.; Nimatoudis, I.; lacovides, A.; Kaprinis, G. Off-label indications for atypical antipsychotics: A systematic review. Ann. Gen. Hosp. Psychiatry. 2004, 3 (1), 4.

10. Mathews, M.; Muzina, D. J. Atypical antipsychotics: new drugs, new challenges. Cleve Clin J Med. 2007, 74 (8), 597-606.

11. Ader, M.; Kim, S. P.; Catalano, K. J.; Ionut, V.; Hucking, K.; Richey, J. M.; Kabir, M.; Bergman, R. N. Metabolic dysregulation with atypical antipsychotics occurs in the absence of underlying disease: a placebo-controlled study of olanzapine and risperidone in dogs. Diabetes. 2005, 54 (3), 862-871.

12. Vidarsdottir, S.; de Leeuw van Weenen JE; Frolich, M.; Roelfsema, F.; Romijn, J. A.; Pijl, H. Effects of olanzapine and haloperidol on the metabolic status of healthy men. J Clin Endocrinol Metab. 2010, 95 (1), 118-125.

13. Allison, D. B.; Casey, D. E. Antipsychotic-induced weight gain: a review of the literature. J Clin Psychiatry 2001, 62 (Suppl 7), 22-31.

14. Citrome, L.; Holt, R. I.; Walker, D. J.; Hoffmann, V. P. Weight gain and changes in metabolic variables following olanzapine treatment in schizophrenia and bipolar disorder. Clin Drug Investig. 2011, 31 (7), 455-482.

15. Eli Lilly and Company Highlights of prescribing information. pi. lilly. com/us/zyprexa-pi. pdf 2011.

16. Weiden, P. J.; Mackell, J. A.; McDonnell, D. D. Obesity as a risk factor for antipsychotic noncompliance. Schizophr Res. 2004, 66 (1), 51-57.

17. Baptista, T.; Zarate, J.; Joober, R.; Colasante, C.; Beaulieu, S.; Paez, X.; Hernandez, L. Drug induced weight gain, an impediment to successful pharmacotherapy: focus on antipsychotics. Curr Drug Targets. 2004, 5 (3), 279-299.

18. Mojtabai, R.; Lavelle, J.; Gibson, P. J.; Bromet, E. J. Atypical antipsychotics in first admission schizophrenia: medication continuation and outcomes. Schizophr Bull. 2003, 29 (3), 519-530.

19. Dilla, T.; Ciudad, A.; Alvarez, M. Systematic review of the economic aspects of nonadherence to antipsychotic medication in patients with schizophrenia. Patient. Prefer. Adherence. 2013, 7:275-84. doi: 10.2147/PPA.S41609. Print;\%2013., 275-284.

20. Weiden, P. J.; Olfson, M. Cost of relapse in schizophrenia. Schizophr Bull. 1995, 21 (3), 419-429. 
21. Swinburn, B. A.; Sacks, G.; Hall, K. D.; McPherson, K.; Finegood, D. T.; Moodie, M. L.; Gortmaker, S. L. The global obesity pandemic: shaped by global drivers and local environments. Lancet 2011, 378 (9793), 826-837.

22. Flegal, K. M.; Carroll, M. D.; Ogden, C. L.; Curtin, L. R. Prevalence and trends in obesity among US adults, 1999-2008. JAMA. 2010, 303 (3), 235-241.

23. Flegal, K. M.; Carroll, M. D.; Kuczmarski, R. J.; Johnson, C. L. Overweight and obesity in the United States: prevalence and trends, 19601994. Int. J. Obes. Relat Metab Disord. 1998, 22 (1), 39-47.

24. Bhala, N.; Angulo, P.; van der Poorten, D.; Lee, E.; Hui, J. M.; Saracco, G.; Adams, L. A.; Charatcharoenwitthaya, P.; Topping, J. H.; Bugianesi, E.; Day, C. P.; George, J. The natural history of nonalcoholic fatty liver disease with advanced fibrosis or cirrhosis: an international collaborative study. Hepatology. 2011, 54 (4), 1208-1216.

25. Ludwig, J.; Viggiano, T. R.; McGill, D. B.; Oh, B. J. Nonalcoholic steatohepatitis: Mayo Clinic experiences with a hitherto unnamed disease. Mayo Clin. Proc. 1980, 55 (7), 434-438.

26. Day, C. P. Non-alcoholic fatty liver disease: current concepts and management strategies. Clin. Med. 2006, 6 (1), 19-25.

27. Ratziu, V.; Bonyhay, L.; Di, M., V; Charlotte, F.; Cavallaro, L.; SayeghTainturier, M. H.; Giral, P.; Grimaldi, A.; Opolon, P.; Poynard, T. Survival, liver failure, and hepatocellular carcinoma in obesityrelated cryptogenic cirrhosis. Hepatology. 2002, 35 (6), 1485-1493.

28. Masuoka, H. C.; Chalasani, N. Nonalcoholic fatty liver disease: an emerging threat to obese and diabetic individuals. Ann. N. Y. Acad Sci. 2013, 1281:106-22. doi: 10.1111/nyas.12016. Epub;\%2013 Jan 30., 106-122.

29. Lazo, M.; Hernaez, R.; Eberhardt, M. S.; Bonekamp, S.; Kamel, I.; Guallar, E.; Koteish, A.; Brancati, F. L.; Clark, J. M. Prevalence of Nonalcoholic Fatty Liver Disease in the United States: The Third National Health and Nutrition Examination Survey, 1988-1994. Am J Epidemiol. 2013.

30. Ogden, C. L.; Carroll, M. D.; Curtin, L. R.; McDowell, M. A.; Tabak, C. J.; Flegal, K. M. Prevalence of overweight and obesity in the United States, 1999-2004. JAMA. 2006, 295 (13), 1549-1555. 
31. Heiskanen, T.; Niskanen, L.; Lyytikainen, R.; Saarinen, P. I.; Hintikka, J. Metabolic syndrome in patients with schizophrenia. J Clin Psychiatry. 2003, 64 (5), 575-579.

32. Allison, D. B.; Newcomer, J. W.; Dunn, A. L.; Blumenthal, J. A.; Fabricatore, A. N.; Daumit, G. L.; Cope, M. B.; Riley, W. T.; Vreeland, B.; Hibbeln, J. R.; Alpert, J. E. Obesity among those with mental disorders: a National Institute of Mental Health meeting report. Am J Prev. Med. 2009, 36 (4), 341-350.

33. Dickerson, F. B.; Brown, C. H.; Kreyenbuhl, J. A.; Fang, L.; Goldberg, R. W.; Wohlheiter, K.; Dixon, L. B. Obesity among individuals with serious mental illness. Acta Psychiatr Scand. 2006, 113 (4), 306313.

34. Haberfellner, E. M.; Honsig, T. Nonalcoholic steatohepatitis: a possible side effect of atypical antipsychotics. J Clin Psychiatry. 2003, 64 (7), 851.

35. Arulmozhi, D. K.; Dwyer, D. S.; Bodhankar, S. L. Antipsychotic induced metabolic abnormalities: an interaction study with various PPAR modulators in mice. Life Sci. 2006, 79 (19), 1865-1872.

36. Chintoh, A. F.; Mann, S. W.; Lam, L.; Lam, C.; Cohn, T. A.; Fletcher, P. J.; Nobrega, J. N.; Giacca, A.; Remington, G. Insulin resistance and decreased glucose-stimulated insulin secretion after acute olanzapine administration. J Clin Psychopharmacol. 2008, 28 (5), 494-499.

37. McElroy, S. L. Obesity in patients with severe mental illness: overview and management. J Clin Psychiatry. 2009, 70 Suppl 3:12-21. doi: 10.4088/JCP.7075su1C.03., 12-21.

38. Langhans, W. Role of the liver in the control of glucose-lipid utilization and body weight. Curr Opin Clin Nutr Metab Care. 2003, 6 (4), 449-455.

39. Fabbrini, E.; Sullivan, S.; Klein, S. Obesity and nonalcoholic fatty liver disease: biochemical, metabolic, and clinical implications. Hepatology. 2010, 51 (2), 679-689.

40. Aravagiri, M.; Teper, Y.; Marder, S. R. Pharmacokinetics and tissue distribution of olanzapine in rats. Biopharm. Drug Dispos. 1999, 20 (8), 369-377.

41. Sandoval, D. A.; Obici, S.; Seeley, R. J. Targeting the CNS to treat type 2 diabetes. Nat Rev Drug Discov. 2009, 8 (5), 386-398. 
42. Weston-Green, K.; Huang, X. F.; Deng, C. Alterations to melanocortinergic, GABAergic and cannabinoid neurotransmission associated with olanzapine-induced weight gain. PLOS One. 2012, 7 (3), e33548.

43. Grayson, B. E.; Seeley, R. J.; Sandoval, D. A. Wired on sugar: the role of the CNS in the regulation of glucose homeostasis. Nat Rev Neurosci. 2013, 14 (1), 24-37.

44. Girault, E. M.; Alkemade, A.; Foppen, E.; Ackermans, M. T.; Fliers, E.; Kalsbeek, A. Acute peripheral but not central administration of olanzapine induces hyperglycemia associated with hepatic and extra-hepatic insulin resistance. PLoS One. 2012, 7 (8), e43244.

45. Chintoh, A. F.; Mann, S. W.; Lam, T. K.; Giacca, A.; Remington, G. Insulin resistance following continuous, chronic olanzapine treatment: An animal model. Schizophr Res. 2008, 104, 23-30.

46. Machado, M.; Marques-Vidal, P.; Cortez-Pinto, H. Hepatic histology in obese patients undergoing bariatric surgery. J. Hepatol. 2006, 45 (4), 600-606.

47. Day, C. P.; James, O. F. Steatohepatitis: a tale of two "hits"? Gastroenterology. 1998, 114 (4), 842-845.

48. Yang, S. Q.; Lin, H. Z.; Lane, M. D.; Clemens, M.; Diehl, A. M. Obesity increases sensitivity to endotoxin liver injury: implications for the pathogenesis of steatohepatitis. Proc Natl Acad Sci U S A. 1997, 94, 2557-2562.

49. Kregel, K. C.; Zhang, H. J. An integrated view of oxidative stress in aging: basic mechanisms, functional effects, and pathological considerations. Am J Physiol Regul. Integr. Comp Physiol. 2007, 292 (1), R18-R36.

50. Arteel, G. E. Oxidants and antioxidants in alcohol-induced liver disease. Gastroenterology 2003, 124 (3), 778-790.

51. Pi, J.; Zhang, Q.; Fu, J.; Woods, C.; Hou, Y.; Corkey, B.; Collins, S.; Andersen, M. ROS signaling, oxidative stress and Nrf2 in pancreatic beta-cell function. Tox. Appl. Pharmacol. 2010, 244 (1), 77-83.

52. Sies, H. Oxidative Stress: Oxidants and Antioxidants. Exp Physiol 1997, 82, 291-295. 
53. Klaasen, C. D.; Reisman, S. A. Nrf2 the rescue: Effects of the antioxidative/electrophilic response on the liver. Tox. Appl.

Pharmacol. 2010, 244, 57-65.

54. Maher, J.; Yamamoto, M. The rise of antioxidant signaling--the evolution and hormetic actions of Nrf2. Toxicol. Appl Pharmacol. 2010, 244 (1), 4-15.

55. Kensler, T. W.; Wakabayashi, N.; Biswal, S. Cell survival responses to environmental stresses via the Keap1-Nrf2-ARE pathway. Annu. Rev Pharmacol Toxicol. 2007, 47:89-116., 89-116.

56. Kohen, R.; Nyska, A. Oxidation of biological systems: oxidative stress phenomena, antioxidants, redox reactions, and methods for their quantification. Toxicol. Pathol. 2002, 30 (6), 620-650.

57. Ohara, Y.; Peterson, T. E.; Harrison, D. G. Hypercholesterolemia Increases Endothelial Superoxide Anion Production. J Clin Invest 1993, 91, 2546-2551.

58. Inoguchi, T.; Li, P.; Umeda, F.; Yu, H. Y.; Kakimoto, M.; Imamura, M.; Aoki, T.; Etoh, T.; Hashimoto, T.; Naruse, M.; Sano, H.; Utsumi, H.; Nawata, H. High Glucose Level and Free Fatty Acid Stimulate Reactive Oxygen Species Production Through Protein Kinase CDependent Activation of NAD $(\mathrm{P}) \mathrm{H}$ Oxidase in Cultured Vascular Cells. Diabetes 2000, 49, 1939-1945.

59. Brownlee, M. Biochemistry and molecular cell biology of diabetic complications. Nature 2001, 414, 813-820.

60. Pessayre, D.; Fromenty, B. NASH: a mitochondrial disease. J. Hepatol. 2005, 42, 928-940.

61. Browning, J. D.; Horton, J. D. Molecular mediators of hepatic steatosis and liver injury. J Clin Invest 2004, 114 (2), 147-152.

62. Padurariu, M.; Ciobica, A.; Dobrin, I.; Stefanescu, C. Evaluation of antioxidant enzymes activities and lipid peroxidation in schizophrenic patients treated with typical and atypical antipsychotics. Neurosci. Lett. 2010, 479 (3), 317-320.

63. Lu, S. C. Antioxidants in the treatment of chronic liver diseases: why is the efficacy evidence so weak in humans? Hepatology 2008, 48 (5), 1359-1361.

64. Podmore, I.; Griffiths, H.; Herbert, K.; Mistry, N.; Mistry, P.; Lunec, J. Vitamin C exhibits pro-oxidant properties. Nature 1998, 392, 559. 
65. Paolini, M.; Pozzetti, L.; Pedulli, G. F.; Marchesi, E.; Cantelli-Forti, G. The Nature of Prooxidant Activity of Vitamin C. Life Sci. 1999, 64 (23), 273-278.

66. Miller, E. R. 3.; Pastor-Barriuso, R.; Dalal, D.; Riemersma, R.; Appel, L. J.; Guallar, E. Meta-analysis: high-dosage vitamin E supplementation may increase all-cause mortality. Ann. Intern. Med. 2005, 142 (1), $37-46$.

67. Sies, H. Strategies of antioxidant defense. Eur. J. Biochem. 1993, 215, 213-219.

68. Steinhubl, S. R. Why Have Antioxidants Failed in Clinical Trials? Am J Cardiol 2008, 101 (10A), 14D-19D.

69. Dinkova-Kostova, A. T.; Talalay, P. Direct and indirect antioxidant properties of inducers of cytoprotective proteins. Mol. Nutr. Food Res. 2008, 52, S128-S138.

70. Fahey, J. W.; Talalay, P. Antioxidant functions of sulforaphane: a potent inducer of Phase II detoxication enzymes. Food Chem. Toxicol. 1999, 37 (9-10), 973-979.

71. Juge, N.; Mithen, R. F.; Traka, M. Molecular basis for chemoprevention by sulforaphane: a comprehensive review. Cell. Mol. Life Sci. 2007, 64 (9), 1105-1127.

72. Salmasi, F. B.; Jazayeri, M.; Ghaeli, P.; Hashemian, F.; Akhondzadeh, S.; Raisi, F.; Hosseini, S. H.; Setareh, M. J.; Khavidaki, S. D. Comparing the effects of high-dose vitamin $E$ with those of placebo on insulin resistance in patients with schizophrenia treated with olanzapine. J Clin Psychopharmacol. 2009, 29 (2), 182-183.

73. Westerlund, C.; Ostlund-Lindqvist, A. M.; Sainsbury, M.; Shertzer, H. G.; Sjoquist, P. O. Characterization of novel indenoindoles. Part I. Structure-activity relationships in different model systems of lipid peroxidation. Biochem Pharmacol. 1996, 51 (10), 1397-1402.

74. Shertzer, H.; Kendig, E.; Nasrallah, H.; Johansson, E.; Genter, M. Protection from olanzapine-induced metabolic toxicity in mice by acetaminophen and tetrahydroindenoindole. Int J Obes 2010, 34 (6), 970-979.

75. Mas, S.; Gasso, P.; Trias, G.; Bernardo, M.; Lafuente, A. Sulforaphane protects SK-N-SH cells against antipsychotic-induced oxidative stress. Fundam Clin Pharmacol. 2012, 26 (6), 712-721. 
76. Kozul, C. D.; Nomikos, A. P.; Hampton, T. H.; Warnke, L. A.; Gosse, J. A.; Davey, J. C.; Thorpe, J. E.; Jackson, B. P.; Ihnat, M. A.; Hamilton, J. W. Laboratory diet profoundly alters gene expression and confounds genomic analysis in mouse liver and lung. Chem. Biol. Interact. 2008, 173 (2), 129-140.

77. Kapur, S.; VanderSpek, S. C.; Brownlee, B. A.; Nobrega, J. N. Antipsychotic dosing in preclinical models is often unrepresentative of the clinical condition: a suggested solution based on in vivo occupancy. J Pharmacol Exp. Ther. 2003, 305 (2), 625-631.

78. McDonnell, D. P.; Kryzhanovskaya, L. A.; Zhao, F.; Detke, H. C.; Feldman, P. D. Comparison of metabolic changes in patients with schizophrenia during randomized treatment with intramuscular olanzapine long-acting injection versus oral olanzapine. Hum Psychopharmacol. 2011, 26 (6), 422-433.

79. van der Zwaal, E. M.; Luijendijk, M. C.; Adan, R. A.; la Fleur, S. E. Olanzapine-induced weight gain: chronic infusion using osmotic minipumps does not result in stable plasma levels due to degradation of olanzapine in solution. Eur J Pharmacol. 2008, 585 (1), 130-136.

80. Salamone, L. M.; Fuerst, T.; Kern, M.; Lang, T.; Dockrell, M.; Cauley, J. A.; Nevitt, M.; Tylavsky, F.; Lohman, T. G. Measurement of fat mass using DEXA: a validation study in elderly adults. J Appl Physiol. 2000, 89 (1), 345-352.

81. Andrikopoulos, S.; Blair, A. R.; Deluca, N.; Fam, B. C.; Proietto, J. Evaluating the glucose tolerance test in mice. Am J Physiol Endocrinol Metab. 2008, 295 (6), E1323-E1332.

82. Bligh, E. G.; Dyer, W. J. A rapid method of total lipid extraction and purification. Can. J. Biochem. Physiol. 1959, 37, 911-917.

83. Arteel, G. E.; limuro, Y.; Yin, M.; Raleigh, J. A.; Thurman, R. G. Chronic enteral ethanol treatment causes hypoxia in rat liver tissue in vivo. Hepatology 1997, 25 (4), 920-926.

84. Arteel, G. E.; Gaebele, E.; Wheeler, M. D.; Uesugi, T.; Kadiiska, M.; Connor, H. D.; Mason, R. P.; Thurman, R. G. Inducible nitric oxide synthase is necessary for alcohol-induced liver injury: studies with knock-out mice. Hepatology 2003, 34, 339A.

85. von Montfort, C.; Beier, J. I.; Guo, L.; Kaiser, J. P.; Arteel, G. E. Contribution of the sympathetic hormone epinephrine to the sensitizing effect of ethanol on LPS-induced liver damage in mice. 
Am. J. Physiol Gastrointest. Liver Physiol. 2008, 294 (5), G1227G1234.

86. SEIFTER, S.; DAYTON, S.; Novic, B.; Muntwyler, E. The estimation of glycogen with the anthrone reagent. Arch Biochem. 1950, 25 (1), 191-200.

87. Leyva, A.; Quintana, A.; Sanchez, M.; Rodriguez, E. N.; Cremata, J.; Sanchez, J. C. Rapid and sensitive anthrone-sulfuric acid assay in microplate format to quantify carbohydrate in biopharmaceutical products: method development and validation. Biologicals. 2008, 36 (2), 134-141.

88. Warburg, O.; Geissler, A. W.; Lorenz, S. Uber Wachstum von Krebszellen in Medien, deren Glucose durch Galaktose ersetzt ist. Hoppe Seylers. Z Physiol Chem. 1967, 348 (12), 1686-1687.

89. Wei, X.; Shi, X.; Koo, I.; Kim, S.; Schmidt, R. H.; Arteel, G. E.; Watson, W. H.; McClain, C.; Zhang, X. MetPP: a computational platform for comprehensive two-dimensional gas chromatography time-of-flight mass spectrometry-based metabolomics. Bioinformatics. 2013.

90. Zhang, J.; Fang, A.; Wang, B.; Kim, S. H.; Bogdanov, B.; Zhou, Z.; McClain, C.; Zhang, $X$. iMatch: a retention index tool for analysis of gas chromatography-mass spectrometry data. J Chromatogr. A. 2011, 1218 (37), 6522-6530.

91. Cope, M. B.; Nagy, T. R.; Fernandez, J. R.; Geary, N.; Casey, D. E.; Allison, D. B. Antipsychotic drug-induced weight gain: development of an animal model. Int J Obes 2005, 29, 607-614.

92. Hroudova, J.; Fisar, Z. Activities of respiratory chain complexes and citrate synthase influenced by pharmacologically different antidepressants and mood stabilizers. Neuroendocrinology Letters 2010, 31 (3), 336-342.

93. Marroquin, L. D.; Hynes, J.; Dykens, J. A.; Jamieson, J. D.; Will, Y. Circumventing the Crabtree effect: replacing media glucose with galactose increases susceptibility of HepG2 cells to mitochondrial toxicants. Toxicol. Sci. 2007, 97 (2), 539-547.

94. Coccurello, R.; Moles, A. Potential mechanisms of atypical antipsychoticinduced metabolic derangement: Clues for understanding obesity and novel drug design. Pharmacol Therapeut. 2010, 127, 210-251.

95. Hampson, L. J.; Mackin, P.; Agius, L. Stimulation of glycogen synthesis and inactivation of phosphorylase in hepatocytes by serotonergic 
mechanisms, and counter-regulation by atypical antipsychotic drugs. Diabetologia. 2007, 50 (8), 1743-1751.

96. Göhring, I.; Mulder, H. Glutamate dehydrogenase, insulin secretion, and type 2 diabetes: a new means to protect the pancreatic $\beta$-cell? J Endocrinol. 2012, 212 (3), 239-242.

97. Nicklin, P.; Bergman, P.; Zhang, B.; Triantafellow, E.; Wang, H.; Nyfeler, B.; Yang, H.; Hild, M.; Kung, C.; Wilson, C.; Myer, V. E.; MacKeigan, J. P.; Porter, J. A.; Wang, Y. K.; Cantley, L. C.; Finan, P. M.; Murphy, L. O. Bidirectional transport of amino acids regulates mTOR and autophagy. Cell. 2009, 136 (3), 521-534.

98. Evans, K.; Nasim, Z.; Brown, J.; Butler, H.; Kauser, S.; Varoqui, H.; Erickson, J. D.; Herbert, T. P.; Bevington, A. Acidosis-sensing glutamine pump SNAT2 determines amino acid levels and mammalian target of rapamycin signalling to protein synthesis in L6 muscle cells. J Am Soc Nephrol. 2007, 18 (5), 1426-1436.

99. Fuchs, B. C.; Bode, B. P. Amino acid transporters ASCT2 and LAT1 in cancer: partners in crime? Semin. Cancer Biol. 2005, 15 (4), 254266.

100. Almilaji, A.; Pakladok, T.; Guo, A.; Munoz, C.; Foller, M.; Lang, F. Regulation of the glutamate transporter EAAT3 by mammalian target of rapamycin mTOR. Biochem Biophys Res Commun. 2012, 421 (2), 159-163.

101. Goff, D. C.; Hennen, J.; Lyoo, I. K.; Tsai, G.; Wald, L. L.; Evins, A. E.; Yurgelun-Todd, D. A.; Renshaw, P. F. Modulation of brain and serum glutamatergic concentrations following a switch from conventional neuroleptics to olanzapine. Biol Psychiatry. 2002, 51 (6), 493-497.

102. van der Heijden, F. M.; Fekkes, D.; Tuinier, S.; Sijben, A. E.; Kahn, R. S.; Verhoeven, W. M. Amino acids in schizophrenia: evidence for lower tryptophan availability during treatment with atypical antipsychotics? J Neural Transm. 2005, 112 (4), 577-585.

103. Yudkoff, M.; Daikhin, Y.; Nissim, I.; Horyn, O.; Luhovyy, B.; Lazarow, A.; Nissim, I. Brain amino acid requirements and toxicity: the example of leucine. J Nutr. 2005, 135 (6 Suppl), 1531S-1538S.

104. Albaugh, V. L.; Vary, T. C.; Ilkayeva, O.; Wenner, B. R.; Maresca, K. P.; Joyal, J. L.; Breazeale, S.; Elich, T. D.; Lang, C. H.; Lynch, C. J. Atypical antipsychotics rapidly and inappropriately switch peripheral fuel utilization to lipids, impairing metabolic flexibility in rodents. Schizophr Bull. 2012, 38 (1), 153-166. 
105. Dodd, K. M.; Tee, A. R. Leucine and mTORC1: a complex relationship. Am J Physiol Endocrinol Metab. 2012, 302 (11), E1329-E1342.

106. Fox, H. L.; Pham, P. T.; Kimball, S. R.; Jefferson, L. S.; Lynch, C. J. Amino acid effects on translational repressor 4E-BP1 are mediated primarily by L-leucine in isolated adipocytes. Am J Physiol. 1998, 275 (5 Pt 1), C1232-C1238.

107. Krause, U.; Bertrand, L.; Hue, L. Control of p70 ribosomal protein S6 kinase and acetyl-CoA carboxylase by AMP-activated protein kinase and protein phosphatases in isolated hepatocytes. Eur $\mathrm{J}$ Biochem. 2002, 269 (15), 3751-3759.

108. Chan, M. K.; Tsang, T. M.; Harris, L. W.; Guest, P. C.; Holmes, E.; Bahn, $S$. Evidence for disease and antipsychotic medication effects in post-mortem brain from schizophrenia patients. Mol Psychiatry. 2011, 16 (12), 1189-1202.

109. Tsang, C. K.; Qi, H.; Liu, L. F.; Zheng, X. F. Targeting mammalian target of rapamycin (mTOR) for health and diseases. Drug Discov. Today. 2007, $12(3-4), 112-124$.

110. Rosner, M.; Siegel, N.; Valli, A.; Fuchs, C.; Hengstschläger, M. mTOR phosphorylated at $\mathrm{S} 2448$ binds to raptor and rictor. Amino Acids. 2010, 38 (1), 223-228.

111. Hara, H.; Yonezawa, K.; Kozlowski, M. T.; Sugimoto, T.; Andrabi, K.; Weng, Q. P.; Kasuga, M.; Nishimoto, I.; Avruch, J. Regulation of elF-4E BP1 phosphorylation by mTOR. J Biol Chem. 1997, 272 (42), 26457-26463.

112. Guertin, D. A.; Stevens, D. M.; Thoreen, C. C.; Burds, A. A.; Kalaany, N. Y.; Moffat, J.; Brown, M.; Fitzgerald, K. J.; Sabatini, D. M. Ablation in mice of the mTORC components raptor, rictor, or mLST8 reveals that $\mathrm{mTORC2}$ is required for signaling to Akt-FOXO and PKCalpha, but not S6K1. Dev Cell. 2006, 11 (6), 859-871.

113. Hardie, D. G. AMP-activated/SNF1 protein kinases: conserved guardians of cellular energy. Nat Rev Mol Cell Biol. 2008, 8 (10), 774-785.

114. Coccurello, R.; Moles, A. A murine model of atypical antipsychotic-induced weight gain and metabolic dysregulation. 2010; p 9.33.1-9.33.22.

115. Ehret, M.; Goethe, J.; Lanosa, M.; Coleman, C. I. The effect of metformin on anthropometrics and insulin resistance in patients receiving atypical antipsychotic agents: a meta-analysis. J Clin Psychiatry. 2010, 71 (10), 1286-1292. 
116. Laplante, M.; Sabatini, D. M. mTOR signaling in growth control and disease. Cell. 2012, 149 (2), 274-293.

117. Xu, G.; Wang, Z.; Li, Y.; Li, Z.; Tang, H.; Zhao, J.; Xiang, X.; Ding, L.; Ma, L.; Yuan, F.; Fei, J.; Wang, W.; Wang, N.; Guan, Y.; Tang, C.; Mulholland, M.; Zhang, W. Ghrelin contributes to derangements of glucose metabolism induced by rapamycin in mice. Diabetologia. 2012, 55 (6), 1813-1823.

118. Aggarwal, D.; Fernandez, M. L.; Soliman, G. A. Rapamycin, an mTOR inhibitor, disrupts triglyceride metabolism in guinea pigs. Metabolism. 2006, 55 (6), 794-802.

119. Zhao, L.; Ding, T.; Cyrus, T.; Cheng, Y.; Tian, H.; Ma, M.; Falotico, R.; Pratico, D. Low-dose oral sirolimus reduces atherogenesis, vascular inflammation and modulates plaque composition in mice lacking the LDL receptor. Br J Pharmacol. 2009, 156 (5), 774-785.

120. Chang, G. R.; Chiu, Y. S.; Wu, Y. Y.; Chen, W. Y.; Liao, J. W.; Chao, T. H.; Mao, F. C. Rapamycin protects against high fat diet-induced obesity in C57BL/6J mice. J Pharmacol Sci. 2009, 109 (4), 496503.

121. Neef, M.; Ledermann, M.; Saegesser, H.; Schneider, V.; Reichen, J. Lowdose oral rapamycin treatment reduces fibrogenesis, improves liver function, and prolongs survival in rats with established liver cirrhosis. J Hepatol. 2006, 45 (6), 786-796.

122. Foster, D. A.; Toschi, A. Targeting mTOR with rapamycin: one dose does not fit all. Cell Cycle. 2009, 8 (7), 1026-1029.

123. Tsai, G.; Coyle, J. T. Glutamatergic mechanisms in schizophrenia. Annu. Rev Pharmacol Toxicol. 2002, 42:165-79., 165-179.

124. Bechara, C. I.; Goldman-Levine, J. D. Dramatic worsening of type II diabetes mellitus due to olanzapine after 3 years of therapy. Pharmacotherapy. 2001, 21 (11), 1444-1447.

125. Tschoner, A.; Engl, J.; Laimer, M.; Kaser, S.; Rettenbacher, M.; Fleischhacker, W. W.; Patsch, J. R.; Ebenbichler, C. F. Metabolic side effects of antipsychotic medication. Int J Clin Pract. 2007, 61 (8), 1356-1370.

126. Beier, J. I.; Luyendyk, J. P.; Guo, L.; von Montfort, C.; Staunton, D. E.; Arteel, G. E. Fibrin accumulation plays a critical role in the sensitization to lipopolysaccharide-induced liver injury caused by ethanol in mice. Hepatology. 2009, 49 (5), 1545-1553. 
127. Plosker, G. L. Quetiapine: a pharmacoeconomic review of its use in bipolar disorder. Pharmacoeconomics. 2012, 30 (7), 611-631.

128. McIntyre, R. S.; Leiter, L.; Yale, J. F.; CSPQ, M. D.; Lau, D.; Stip, E.; CSPQ, M. M.; Ur11, E.; Marie, J. e.; CPMSQ, M. F. Schizophrenia, glycemia and antipsychotic medications: an expert consensus review. Can J Diabetes 2005, 29, 113-121.

129. Fimognari, C.; Lenzi, M.; Hrelia, P. Interaction of the isothiocyanate sulforaphane with drug disposition and metabolism: pharmacological and toxicological implications. Curr Drug Metab. 2008, 9 (7), 668-678.

130. Loignon, M.; Miao, W.; Hu, L.; Bier, A.; Bismar, T. A.; Scrivens, P. J.; Mann, K.; Basik, M.; Bouchard, A.; Fiset, P. O.; Batist, Z.; Batist, G. Cul3 overexpression depletes Nrf2 in breast cancer and is associated with sensitivity to carcinogens, to oxidative stress, and to chemotherapy. Mol Cancer Ther. 2009, 8 (8), 2432-2440.

131. Dandona, P.; Ghanim, H.; Chaudhuri, A.; Dhindsa, S.; Kim, S. S. Macronutrient intake induces oxidative and inflammatory stress: potential relevance to atherosclerosis and insulin resistance. Exp. Mol Med. 2010, 42 (4), 245-253.

132. Brown, L. A.; Kerr, C. J.; Whiting, P.; Finer, N.; McEneny, J.; Ashton, T. Oxidant stress in healthy normal-weight, overweight, and obese individuals. Obesity. (Silver. Spring). 2009, 17 (3), 460-466.

133. Schmidt, A. J.; Hemmeter, U. M.; Krieg, J. C.; Vedder, H.; Heiser, P. Impact of haloperidol and quetiapine on the expression of genes encoding antioxidant enzymes in human neuroblastoma SH-SY5Y cells. J Psychiatr Res. 2009, 43 (8), 818-823.

134. Heiser, P.; Sommer, O.; Schmidt, A. J.; Clement, H. W.; Hoinkes, A.; Hopt, U. T.; Schulz, E.; Krieg, J. C.; Dobschutz, E. Effects of antipsychotics and vitamin $\mathrm{C}$ on the formation of reactive oxygen species. J Psychopharmacol. 2010, 24 (10), 1499-1504.

135. Eroglu, S.; Pandir, D.; Uzun, F. G.; Bas, H. Protective role of vitamins C and $E$ in diclorvos-induced oxidative stress in human erythrocytes in vitro. Biol Res. 2013, 46 (1), 33-38.

136. Atkinson, D. E.; Fall, L. Adenosine triphosphate conservation in biosynthetic regulation. Escherichia coli phosphoribosylpyrophosphate synthase. J Biol Chem. 1967, 242 (13), 3241-3242. 
137. Lin, H. Z.; Yang, S. Q.; Chuckaree, C.; Kuhajda, F.; Ronnet, G.; Diehl, A. $M$. Metformin reverses fatty liver disease in obese, leptin-deficient mice. Nat. Med. 2000, 6 (9), 998-1003.

138. Martins, P. J.; Haas, M.; Obici, S. Central nervous system delivery of the antipsychotic olanzapine induces hepatic insulin resistance. Diabetes. 2010, 59 (10), 2418-2425.

139. Kast, R. E.; Karpel-Massler, G.; Halatsch, M. E. Can the therapeutic effects of temozolomide be potentiated by stimulating AMPactivated protein kinase with olanzepine and metformin? $\mathrm{Br} \mathrm{J}$ Pharmacol. 2011, 164 (5), 1393-1396.

140. Kim, S. F.; Huang, A. S.; Snowman, A. M.; Teuscher, C.; Snyder, S. H. Antipsychotic drug-induced weight gain mediated by histamine $\mathrm{H} 1$ receptor-linked activation of hypothalamic AMP-kinase. Proc Natl Acad Sci U S A. 2007, 104 (9), 3456-3459.

141. Meltzer, H. Y. Illuminating the molecular basis for some antipsychotic drug-induced metabolic burden. Proc Natl Acad Sci U S A. 2007, 104 (9), 3019-3020.

142. Boyle, J. G.; Salt, I. P.; McKay, G. A. Metformin action on AMP-activated protein kinase: a translational research approach to understanding a potential new therapeutic target. Diabet. Med. 2010, 27 (10), 1097-1106.

143. Housel, A. K.; Waterbury, N.; Argo, T. R. Can metformin or rosiglitazone reduce metabolic side effects associated with atypical antipsychotics? Issues Ment Health Nurs. 2009, 30 (12), 803-805.

144. Kimball, S. R. Interaction between the AMP-activated protein kinase and mTOR signaling pathways. Med Sci Sports Exerc. 2006, 38 (11), 1958-1964.

145. Rosner, M.; Fuchs, C.; Siegel, N.; Valli, A.; Hengstschläger, M. Functional interaction of mammalian target of rapamycin complexes in regulating mammalian cell size and cell cycle. Hum Mol Genet. 2009, 18 (17), 3298-3310.

146. Blanchard, P. G.; Festuccia, W. T.; Houde, V. P.; St-Pierre, P.; Brule, S.; Turcotte, V.; Cote, M.; Bellmann, K.; Marette, A.; Deshaies, Y. Major involvement of mTOR in the PPARgamma-induced stimulation of adipose tissue lipid uptake and fat accretion. $J$ Lipid Res. 2012, 53 (6), 1117-1125.

147. Shah, O. J.; Wang, Z.; Hunter, T. Inappropriate activation of the TSC/Rheb/mTOR/S6K cassette induces IRS1/2 depletion, insulin 
resistance, and cell survival deficiencies. Curr Biol. 2004, 14 (18), 1650-1656.

148. Jansson, N.; Rosario, F. J.; Gaccioli, F.; Lager, S.; Jones, H. N.; Roos, S.; Jansson, T.; Powell, T. L. Activation of placental mTOR signaling and amino acid transporters in obese women giving birth to large babies. J Clin Endocrinol Metab. 2013, 98 (1), 105-113.

149. Hoeffer, C. A.; Klann, E. mTOR signaling: At the crossroads of plasticity, memory and disease. Trends Neurosci. 2010, 33 (2), 67-75.

150. Siuta, M. A.; Robertson, S. D.; Kocalis, H.; Saunders, C.; Gresch, P. J.; Khatri, V.; Shiota, C.; Kennedy, J. P.; Lindsley, C. W.; Daws, L. C.; Polley, D. B.; Veenstra-Vanderweele, J.; Stanwood, G. D.; Magnuson, M. A.; Niswender, K. D.; Galli, A. Dysregulation of the norepinephrine transporter sustains cortical hypodopaminergia and schizophrenia-like behaviors in neuronal rictor null mice. PLOS Biol. 2010, 8 (6), e1000393.

151. Bou Khalil, R. Is there any place for macrolides in mood disorders? Med Hypotheses. 2012, 78 (1), 86-87.

152. Cusin, C.; Hilton, G. Q.; Nierenberg, A. A.; Fava, M. Long-term maintenance with intramuscular ketamine for treatment-resistant bipolar II depression. Am J Psychiatry. 2012, 169 (8), 868-869.

153. Sienaert, P.; Lambrichts, L.; Dols, A.; De Fruyt, J. Evidence-based treatment strategies for treatment-resistant bipolar depression: a systematic review. Bipolar Disord. 2013, 15 (1), 61-69.

154. Nguyen, T.; Nioi, P.; Pickett, C. B. The Nrf2-antioxidant response element signaling pathway and its activation by oxidative stress. $\mathrm{J}$ Biol Chem. 2009, 284 (20), 13291-13295.

155. Surh, Y. J.; Kundu, J. K.; Na, H. K. Nrf2 as a master redox switch in turning on the cellular signaling involved in the induction of cytoprotective genes by some chemopreventive phytochemicals. Planta Med. 2008, 74 (13), 1526-1539.

156. Zhang, D. D.; Hannink, M. Distinct cysteine residues in Keap1 are required for Keap1-dependent ubiquitination of Nrf2 and for stabilization of Nrf2 by chemopreventive agents and oxidative stress. Mol Cell Biol. 2003, 23 (22), 8137-8151.

157. Yamamoto, T.; Suzuki, T.; Kobayashi, A.; Wakabayashi, J.; Maher, J.; Motohashi, H.; Yamamoto, M. Physiological significance of reactive cysteine residues of Keap1 in determining Nrf2 activity. Mol Cell Biol. 2008, 28 (8), 2758-2770. 
158. Rushmore, T. H.; Morton, M. R.; Pickett, C. B. The antioxidant responsive element. Activation by oxidative stress and identification of the DNA consensus sequence required for functional activity. $J$ Biol Chem. 1991, 266 (18), 11632-11639.

159. Purdom-Dickinson, S. E.; Sheveleva, E. V.; Sun, H.; Chen, Q. M. Translational control of nrf2 protein in activation of antioxidant response by oxidants. Mol Pharmacol. 2007, 72 (4), 1074-1081.

160. Shibata, T.; Saito, S.; Kokuba, A.; Suzuki, T.; Yamamoto, M.; Hirohashi, S. Global downstream pathway analysis reveals a dependence of oncogenic NF-E2-related factor 2 mutation on the mTOR growth signaling pathway. Cancer Res. 2010, 70 (22), 9095-9105.

161. Liby, K. T.; Sporn, M. B. Synthetic oleanane triterpenoids: multifunctional drugs with a broad range of applications for prevention and treatment of chronic disease. Pharmacol Rev. 2012, 64 (4), 9721003.

162. Lepping, P.; Sambhi, R. S.; Whittington, R.; Lane, S.; Poole, R. Clinical relevance of findings in trials of antipsychotics: systematic review. Br J Psychiatry. 2011, 198 (5), 341-345.

163. Komossa, K.; Rummel-Kluge, C.; Hunger, H.; Schmid, F.; Schwarz, S.; Duggan, L.; Kissling, W.; Leucht, S. Olanzapine versus other atypical antipsychotics for schizophrenia. Cochrane Database Syst Rev. 2010, (3), CD006654.

164. Guertin, D. A.; Sabatini, D. M. The pharmacology of mTOR inhibition. Sci Signal. 2009, 2 (67), e24.

165. Krause, U.; Bertrand, L.; Hue, L. Control of $p 70$ ribosomal protein S6 kinase and acetyl-CoA carboxylase by AMP-activated protein kinase and protein phosphatases in isolated hepatocytes. Eur J Biochem. 2002, 269 (15), 3751-3759.

166. Oh, K. J.; Park, J.; Lee, S. Y.; Hwang, I.; Kim, J. B.; Park, T. S.; Lee, H. J.; Koo, S. H. Atypical antipsychotic drugs perturb AMPK-dependent regulation of hepatic lipid metabolism. Am J Physiol Endocrinol Metab. 2011, 300 (4), E624-E632.

167. Ma, D.; Chan, M. K.; Lockstone, H. E.; Pietsch, S. R.; Jones, D. N.; Cilia, J.; Hill, M. D.; Robbins, M. J.; Benzel, I. M.; Umrania, Y.; Guest, P. C.; Levin, Y.; Maycox, P. R.; Bahn, S. Antipsychotic treatment alters protein expression associated with presynaptic function and nervous system development in rat frontal cortex. J Proteome. Res. 2009, 8 (7), 3284-3297. 
168. He, Y.; Yu, Z.; Giegling, I.; Xie, L.; Hartmann, A. M.; Prehn, C.; Adamski, J.; Kahn, R.; Li, Y.; Illig, T.; Wang-Sattler, R.; Rujescu, D.

Schizophrenia shows a unique metabolomics signature in plasma. Transl. Psychiatry. 2012, 2:e149. doi: 10.1038/tp.2012.76., e149.

169. van der Heijden, F. M.; Tuinier, S.; Fekkes, D.; Sijben, A. E.; Kahn, R. S.; Verhoeven, W. M. Atypical antipsychotics and the relevance of glutamate and serotonin. Eur Neuropsychopharmacol. 2004, 14 (3), 259-265.

170. Cooper, A. J. Role of glutamine in cerebral nitrogen metabolism and ammonia neurotoxicity. Ment Retard. Dev Disabil. Res Rev. 2001, 7 (4), 280-286.

171. Yudkoff, M.; Daikhin, Y.; Horyn, O.; Nissim, I.; Nissim, I. Ketosis and brain handling of glutamate, glutamine, and GABA. Epilepsia. 2008, 49 Suppl 8:73-5. doi: 10.1111/j.1528-1167.2008.01841.x., 73-75.

172. Bak, L. K.; Schousboe, A.; Waagepetersen, H. S. The glutamate/GABAglutamine cycle: aspects of transport, neurotransmitter homeostasis and ammonia transfer. J Neurochem. 2006, 98 (3), 641-653.

173. McKenna, M. C. The glutamate-glutamine cycle is not stoichiometric: fates of glutamate in brain. J Neurosci. Res. 2007, 85 (15), 3347-3358.

174. Javitt, D. C. Glutamate as a therapeutic target in psychiatric disorders. Mol Psychiatry. 2004, 9 (11), 984-97, 979.

175. Patti, M. E.; Brambilla, E.; Luzi, L.; Landaker, E. J.; Kahn, C. R. Bidirectional modulation of insulin action by amino acids. J Clin Invest. 1998, 101 (7), 1519-1529.

176. Tato, I.; Bartrons, R.; Ventura, F.; Rosa, J. L. Amino acids activate mammalian target of rapamycin complex 2 (mTORC2) via PI3K/Akt signaling. J Biol Chem. 2011, 286 (8), 6128-6142.

177. Dominguez-Jimenez, J. L.; Puente-Gutierrez, J. J.; Pelado-Garcia, E. M.; Cuesta-Cubillas, D.; Garcia-Moreno, A. M. Liver toxicity due to olanzapine. Rev Esp Enferm Dig. 2012, 104 (11), 617-618.

178. Kobayashi, A.; Kang, M. I.; Watai, Y.; Tong, K. I.; Shibata, T.; Uchida, K.; Yamamoto, M. Oxidative and electrophilic stresses activate Nrf2 through inhibition of ubiquitination activity of Keap1. Mol Cell Biol. 2006, 26 (1), 221-229.

179. Huang, J.; Tabbi-Anneni, I.; Gunda, V.; Wang, L. Transcription factor Nrf2 regulates SHP and lipogenic gene expression in hepatic lipid 
metabolism. Am J Physiol Gastrointest. Liver Physiol. 2010, 299 (6), G1211-G1221.

180. Kitteringham, N. R.; Abdullah, A.; Walsh, J.; Randle, L.; Jenkins, R. E.; Sison, R.; Goldring, C. E.; Powell, H.; Sanderson, C.; Williams, S.; Higgins, L.; Yamamoto, M.; Hayes, J.; Park, B. K. Proteomic analysis of Nrf2 deficient transgenic mice reveals cellular defence and lipid metabolism as primary Nrf2-dependent pathways in the liver. J Proteomics. 2010, 73 (8), 1612-1631.

181. Serwer, L.; Hashizume, R.; Ozawa, T.; James, C. D. Systemic and local drug delivery for treating diseases of the central nervous system in rodent models. J Vis. Exp. 2010, (42), 1992.

182. Edwards, S.; Koob, G. F. Experimental psychiatric illness and drug abuse models: from human to animal, an overview. Methods Mol Biol. 2012, 829:31-48. doi: 10.1007/978-1-61779-458-2_2., 31-48.

183. Xu, C.; Li, C. Y.; Kong, A. N. Induction of phase I, II and III drug metabolism/transport by xenobiotics. Arch Pharm. Res. 2005, 28 (3), 249-268.

184. Um, S. H.; D'Alessio, D.; Thomas, G. Nutrient overload, insulin resistance, and ribosomal protein S6 kinase 1, S6K1. Cell Metab. 2006, 3 (6), 393-402.

185. Thimmulappa, R. K.; Mai, K. H.; Srisuma, S.; Kensler, T. W.; Yamamoto, M.; Biswal, S. Identification of Nrf2-regulated genes induced by the chemopreventive agent sulforaphane by oligonucleotide microarray. Cancer Res. 2002, 62 (18), 5196-5203.

186. Harris, R. B. Is leptin the parabiotic "satiety" factor? Past and present interpretations. Appetite. 2013, 61 (1), 111-118.

187. Waring, W. S.; Wrate, J.; Bateman, D. N. Olanzapine overdose is associated with acute muscle toxicity. Hum Exp. Toxicol. 2006, 25 (12), 735-740. 


\section{ABBREVIATIONS}

4EBP1

Acly

Akt

ALT

AMPK

AST

CNS

Cpt1a

DEXA

Fasn

FCCP

Gck

Glut1

Glut4

Gsk3b eukaryotic translation initiation factor $4 \mathrm{E}$ binding protein 1

ATP citrate lyase

protein kinase $B$

alanine aminotransferase

AMP-activated protein kinase

aspartate aminotransferase

central nervous system

carnitine palmitoyltransferase 1a

dual-energy x-ray absorptiometry

fatty acid synthase

carbonyl cyanide 4-(trifluoromethoxy)phenylhydrazone

glucokinase

glucose transporter type 1

glucose transporter type 4

glycogen synthase kinase-3 $\beta$ 


\begin{tabular}{|c|c|}
\hline H\&E & hematoxylin and eosin \\
\hline HDL & high-density lipoprotein \\
\hline HFD & high-fat diet; TD.88137 Adjusted Calories diet \\
\hline LAT1 & L-type amino acid transporter 1 \\
\hline LDL & low-density lipoprotein \\
\hline LFD & low-fat diet; TD.08485 Low-Fat Control Diet \\
\hline MTBSTFA & $\mathrm{N}$-(tert-butyldimethylsilyl)-N-methyltrifluoroacetamide \\
\hline mTOR & mammalian target of rapamycin \\
\hline NAFLD & non-alcoholic fatty liver disease \\
\hline NASH & non-alcoholic steatohepatitis \\
\hline NEFA & non-esterified fatty acids \\
\hline OCR & oxygen consumption rate \\
\hline OGTT & oral glucose tolerance test \\
\hline OLZ & olanzapine \\
\hline p70S6K & $70 \mathrm{kDa}$ ribosomal protein s6 kinase \\
\hline Pck1 & phosphoenolpyruvate carboxykinase 1 \\
\hline PAS & periodic acid-Schiff reagent \\
\hline PPR & proton production rate \\
\hline SLC7A5 & solute carrier family 7 member 5 \\
\hline
\end{tabular}


SLC38A2

SNAT2

Srebf1

TBDMSCI

TG

VLDL solute carrier family 38 member 2

system A amino acid transporter 2

sterol regulatory element binding protein 1

tert-butyldimethylchlorosilane

triglycerides

very low-density lipoprotein 


\section{CURRICULUM VITAE}

Robin H. Schmidt

Email: robin.schmidt@louisville.edu

\section{Academic Degrees}

St. John's University

B.S.

2006

Funeral Services

American Academy McAllister Institute

A.Occup.Studies 2006

Funeral Services

University of Louisville

M.S.

2011

Pharmacology and Toxicology

\section{Academic and Professional Experience}

2008-2010

Graduate research fellow, University of Louisville

2010-present

NIEHS T32 predoctoral fellow, University of Louisville

2011-2012

Ohio Valley Society of Toxicology Executive Council 


\section{Membership in Professional Societies}

2010-2012

2010-present

2011-present

2011-present
New York Academy of Sciences

Kentucky Academy of Science

Ohio Valley Society of Toxicology

Society of Toxicology

Organization of Scientific Conferences, Workshops, Symposia, etc.

Organizer, OVSOT Summer Student/Post-Doc Meeting, Louisville, KY

08/11/11, Dept. of Pharmacology and Toxicology and Ohio Valley Society

of Toxicology

Service to the School of Medicine and/or the University

2010-2011 University of Louisville Academic Grievance Committee

\section{Service to the Profession}

See also sections above

2011 Organizer, OVSOT Summer Student/Post-Doc Meeting

2011-2012 Graduate student representative, Executive Council of OVSOT 


\section{Awards and Honors}

2002-2006 Merit scholarship, St. John's University

2002-2006 Dean's list, St. John's University

$2003 \quad$ Phi Eta Sigma Honor Society

2006 Mu Sigma Alpha Honor Society

2006 Graduation with Distinction (cum laude), St. John's

University

2006 Graduation with Distinction (summa cum laude), American

Academy McAllister Institute

2006 Board of Trustees Award, American Academy McAllister

Institute

2008-2010 Graduate research fellowship recipient, Integrated Program in Biomedical Sciences (IPIBS)

2010 Selected for platform presentation, Ohio Valley Society of Toxicology (OVSOT)

2010 Award for platform presentation, 1st place, OVSOT annual meeting

2011-2013 NIEHS T32 pre-doctoral fellowship recipient

$2011 \quad$ Travel award, SOT

2011 Selected for platform presentation, OVSOT 
2012 President's choice award, American Association for the Study of Liver Diseases annual meeting

\section{Bibliography}

\section{A. Published abstracts}

1. Baldauf KJ, Schmidt RH, Ding X, Beier JI, Tan M, Arteel GE (2010) Sulforaphane enhances liver regeneration after partial hepatectomy in mice. Hepatology 52:617A

2. Tan M, Schmidt RH, Beier Jl, Zhong H, States JC, Arteel GE (2011) Chronic low dose arsenic exposure enhances hepatic injury induced by high fat diet in mice. The Toxicologist 120:429

3. Massey VL, Beier Jl, Schmidt R, Zhong H, Arteel GE (2011) Integrin inhibitor cycloRGDfV blunts enhanced LPS-induced liver injury caused by ethanol in mice. Hepatology 54:970A

4. Schmidt RH, Falkner KC, Beier J, Arteel G (2011) Sulforaphane prevents acetaminophen-induced hepatic injury in mice. The Toxicologist 120:99

5. Schmidt RH, Tan M, Jokinen J, Massey VL, Zhong H, Arteel GE (2012) Arsenic enhances experimental liver disease via altering GI tract function: Protection with prebiotics. Hepatology 56:591A 
6. Schmidt R, Tan M, Zhong H, Arteel G (2012) Sulforaphane prevents liver injury caused by high-fat diet and olanzapine. The Toxicologist $126: 78$

\section{B. Papers}

1. Tan M, Schmidt RH, Beier JI, Watson WH, Zhong H, States JC, Arteel GE (2011) Chronic subhepatotoxic exposure to arsenic enhances hepatic injury caused by high fat diet in mice. Toxicol Appl Pharmacol. 257:356-364

2. Wei X, Shi X, Koo I, Kim S, Schmidt RH, Arteel GE, Watson WH, McClain C, Zhang X (2013) MetPP: A Computational Platform for Comprehensive Two-dimensional Gas Chromatography Time-of-flight Mass Spectrometry-based Metabolomics. Bioinformatics. 14:1786-1792

3. Schmidt RH, Jokinen JD, Massey VL, Falkner KC, Shi X, Yin X, Beier JI, Arteel GE (2013) Olanzapine activates hepatic mammalian target of rapamycin (mTOR): new mechanistic insight into metabolic dysregulation with atypical antipsychotics. J Pharmacol Exp Ther. (In press)

4. Shi X, Wei X, Koo I, Schmidt RH, Yin X, Zhang J, Mueller EG, Kim SH, Vaughn A, Arteel GE, McClain CJ, Zhang X, Watson WH (2013) Metabolomic analysis of the effects of chronic arsenic exposure in a mouse model of diet-induced fatty liver disease. (Submitted) 
5. Schmidt RH, Jokinen JD, Massey VL, Falkner KC, Beier JI, Arteel GE (2013) High-fat diet exacerbates olanzapine-induced liver injury. (Manuscript in preparation)

6. Schmidt RH, Kaiser JP, Massey VL, Falkner KC, Beier JI, Arteel GE (2013) Sulforaphane prevents acetaminophen-induced hepatic injury in mice. (Manuscript in preparation)

C. Presentations

Local/Regional

1. Research seminar, 05/2009, "Effects of rapamycin on hepatic steatosis caused by ethanol", University of Louisville, Dept. of Pharmacology and Toxicology, Louisville, KY.

2. Platform session, 09/2010, "Sulforaphane prevents acetaminopheninduced hepatic injury in mice", Ohio Valley Society of Toxicology Annual Meeting, Cincinnati, $\mathrm{OH}$.

3. Poster presentation, 10/2010, "Sulforaphane prevents acetaminophen-induced hepatic injury in mice”, Research! Louisville, Louisville, $\mathrm{KY}$.

4. Oral master's exam, 11/2010, "Sulforaphane prevents acetaminophen-induced liver injury in mice", University of Louisville, Department of Pharmacology and Toxicology, Louisville, KY. 
5. Platform session, 09/2011, "High fat diet exacerbates olanzapineinduced liver injury: protection by sulforaphane", Ohio Valley Society of Toxicology Annual Meeting, Dayton, $\mathrm{OH}$.

6. Poster presentation, 09/2012, "Arsenic enhances experimental liver disease via altering GI tract function: Protection with prebiotics", Research! Louisville, Louisville, KY.

\section{National/International}

7. Poster presentation, $03 / 2011$, "Sulforaphane prevents acetaminophen-induced hepatic injury in mice", Society of Toxicology Annual Meeting, Washington, DC.

8. Poster presentation, $03 / 2012$, "High fat diet exacerbates olanzapine-induced liver injury: protection by sulforaphane", Society of Toxicology Annual Meeting, San Francisco, CA.

9. Poster presentation, 11/2012, "Arsenic enhances experimental liver disease via altering GI tract function: Protection with prebiotics", American Association for the Study of Liver Diseases Annual Meeting, Boston, MA.

\section{Grant and Fellowship Support}

$2008-2010$

Integrated Programs in Biomedical Sciences

Graduate Fellowship

2011-present

NIEHS T32 - ES011564 Training Grant in

Environmental Health 